\title{
A search for clustering around Herbig Ae/Be stars
}

\author{
II. Atlas of the observed sources ${ }^{\star}$ \\ L. Testi ${ }^{1, \star \star}$, F. Palla ${ }^{2}$, and A. Natta ${ }^{2}$ \\ 1 Division of Physics, Mathematics and Astronomy, California Institute of Technology, MS 105-24, Pasadena CA 91125, U.S.A. \\ 2 Osservatorio Astrofisico di Arcetri, Largo E. Fermi 5, I-50125 Firenze, Italy
}

Received March 25; accepted May 11, 1998

\begin{abstract}
We present large field infrared images of a sample of 45 Herbig Ae/Be stars. Stellar parameters, such as age and luminosity, have been derived for all of them in a consistent way. The images have been used to identify stellar groups or clusters associated with the Herbig Ae/Be star. The results presented in this paper form the database for a study of clustering around intermediate mass stars (Testi et al. 1998).
\end{abstract}

Key words: stars: formation - stars: pre-main sequence - infrared: stars

\section{Introduction}

We have conducted an extensive near infrared (NIR) survey of the fields around a large sample of Herbig AeBe stars with the aim of detecting and characterizing the properties of groups of young stars around intermediate mass pre-main sequence stars. We were primarily motivated by the expectation that at NIR wavelenghts, and especially at $K$ band, the reduced extinction would enable the detection of embedded young stars born in the same environment of (and possibly coeval with) the intermediate mass star. Existing NIR surveys are either focused at the detection of close, $d \lesssim 3500$ AU, companions (Leinert

Send offprint requests to: Testi: Caltech, lt@astro.caltech.edu * Based on observations collected at the TIRGO (Gornergrat, Switzerland) operated by the CAISMI-CNR, Firenze, Italy, and at the NOT (La Palma, Canary Islands) operated by the Nordic Optical Telescope Scientific Association (Denmark, Finland, Norway, Sweden).

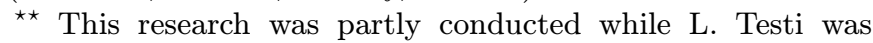
at the Dipartimento di Astronomia e Scienza dello Spazio, Università degli Studi di Firenze, Largo E. Fermi 5, I-50125, Firenze, Italy.

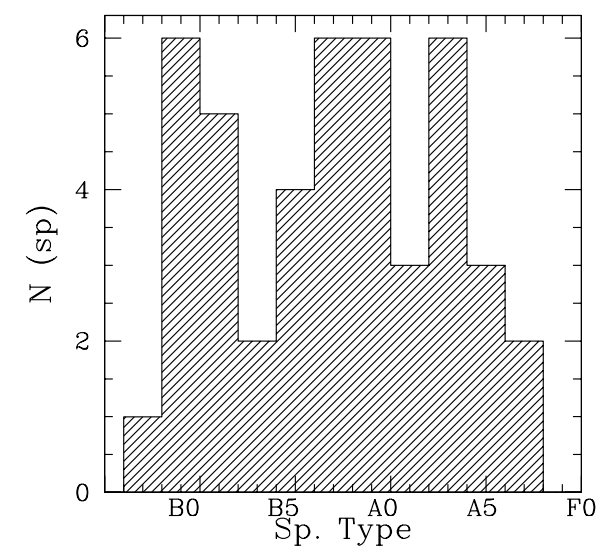

Fig. 1. Distribution of the spectral types of 44 of the observed Herbig AeBe stars (Z CMa, Sp.Type F5, is outside the plot)

et al. 1997; Pirzkal et al. 1997) or are restricted to a limited sample of objects and cover smaller fields than our survey (Li et al. 1994; Hillenbrand 1995).

In Testi et al. (1997; Paper I) we have presented the first results obtained for a subsample of 19 objects. In the present paper we present an atlas of all the fields observed so far (45 total), which forms the database for our study of the clustering around young, intermediate-mass stars (Testi et al. 1998). In Sects. 2 and 3 we describe the sample selection, the observations and data reduction. The results of the observations are presented in Sect. 4, whereas notes on individual sources are given in Sect. 5. We summarize our results in Sect. 6.

\section{Sample selection}

The target stars have been selected from the catalogue of Herbig AeBe stars of Thé et al. (1994) to cover the widest possible range of spectral types and to be easily observable from the northern hemisphere. As shown in Fig. 1, the observed sample covers rather uniformly the 
Table 1. Observed Herbig AeBe stars

\begin{tabular}{|c|c|c|c|c|c|c|c|c|c|c|}
\hline \multicolumn{11}{|c|}{ TIRGO Observations } \\
\hline Star & $\begin{array}{l}\text { R.A. } \\
(1950)\end{array}$ & $\begin{array}{l}\text { Dec. } \\
(1950)\end{array}$ & $\begin{array}{l}\text { Sp. } \\
\text { type }\end{array}$ & $\begin{array}{l}\text { Dist } \\
\text { (pc) }\end{array}$ & $\begin{array}{c}\text { Field } \\
\text { radius }(\mathrm{pc})\end{array}$ & $\underset{J}{\operatorname{Limi}}$ & $\begin{array}{c}\operatorname{ting} 1 \\
H\end{array}$ & $\begin{array}{c}\text { Iagnitude } \\
K\end{array}$ & Acc. & $\begin{array}{r}M_{K}^{\mathrm{c}} \\
(\mathrm{mag})^{a}\end{array}$ \\
\hline Elias 1 & $04: 15: 34.5$ & $+28: 12: 02$ & $\mathrm{~A} 6$ & 160 & 0.16 & 17.6 & 16.5 & 16.4 & $8 \%$ & 9.4 \\
\hline V1012 Ori & 05:09:05.5 & $-02: 26: 24$ & B9 & 460 & 0.47 & 17.8 & 16.7 & 16.3 & $8 \%$ & 7.0 \\
\hline HD 245185 & $05: 32: 24.1$ & $+09: 59: 57$ & A1 & 400 & 0.41 & 17.5 & 16.2 & 16.8 & $7 \%$ & 7.8 \\
\hline MWC 758 & $05: 27: 22.4$ & $+25: 17: 43$ & A3 & 150 & 0.15 & 17.6 & 17.2 & 16.5 & $8 \%$ & 9.6 \\
\hline V380 Ori & $05: 33: 59.5$ & $-06: 44: 46$ & B9 & 450 & 0.46 & 17.0 & 16.6 & 16.5 & $6 \%$ & 7.2 \\
\hline RR Tau & $05: 36: 23.8$ & $+26: 20: 49$ & A3 & 800 & 0.81 & 17.5 & 16.5 & 16.3 & $5 \%$ & 5.8 \\
\hline $\mathrm{LkH} \alpha 208$ & 06:04:53.2 & $+18: 39: 55$ & A3 & 1000 & 1.02 & 17.7 & 17.1 & 16.7 & $5 \%$ & 5.7 \\
\hline VY Mon & $06: 28: 21.0$ & $+10: 28: 15$ & B8 & 800 & 0.81 & 17.7 & 16.9 & 16.4 & $6 \%$ & 5.9 \\
\hline $\mathrm{Z} \mathrm{CMa}$ & $07: 01: 22.5$ & $-11: 28: 36$ & F5 & 1150 & 0.67 & 16.6 & 15.7 & 15.7 & $20 \%$ & 4.4 \\
\hline BHJ 71 & 23:03:07.0 & $+61: 59: 36$ & B0 & 730 & 0.74 & 18.1 & 17.1 & 16.6 & $5 \%$ & 6.3 \\
\hline MWC 1080 & $23: 15: 14.6$ & $+60: 34: 21$ & B0 & 2500 & 1.02 & 17.8 & 16.6 & 16.2 & $5 \%$ & 3.1 \\
\hline \multicolumn{11}{|c|}{ NOT Observations } \\
\hline Star & $\begin{array}{l}\text { R.A. } \\
(1950)\end{array}$ & $\begin{array}{l}\text { Dec. } \\
(1950)\end{array}$ & $\begin{array}{l}\text { Sp. } \\
\text { type }\end{array}$ & $\begin{array}{l}\text { Dist } \\
(\mathrm{pc})\end{array}$ & $\begin{array}{c}\text { Field } \\
\text { radius }(\mathrm{pc})\end{array}$ & \multicolumn{3}{|c|}{$\begin{array}{c}\text { Limiting Magnitude } \\
K\end{array}$} & Acc. & $\begin{array}{r}M_{K}^{\mathrm{c}} \\
(\mathrm{mag})^{a}\end{array}$ \\
\hline MaC H12 & $00: 04: 25.2$ & $+65: 21: 56.9$ & A5 & 850 & 1.05 & \multicolumn{3}{|c|}{17.0} & $3 \%$ & 6.4 \\
\hline VX Cas & $00: 28: 40.4$ & $+61: 42: 17$ & A0 & 760 & 0.43 & \multicolumn{3}{|c|}{17.0} & $3 \%$ & 6.6 \\
\hline RNO 1B & $00: 33: 52.8$ & $+63: 12: 29$ & $\mathrm{Be}$ & 850 & 1.05 & \multicolumn{3}{|c|}{17.0} & $3 \%$ & 6.4 \\
\hline IP Per & $03: 37: 38.5$ & $+32: 22: 16$ & A3 & 350 & 0.43 & \multicolumn{3}{|c|}{17.0} & $3 \%$ & 8.3 \\
\hline MWC 480 & $04: 55: 35.5$ & $+29: 46: 06$ & A2 & 140 & 0.17 & \multicolumn{3}{|c|}{17.0} & $3 \%$ & 10.3 \\
\hline MWC 297 & $18: 25: 01.4$ & $-03: 51: 47$ & O9 & 450 & 0.56 & \multicolumn{3}{|c|}{16.7} & $3 \%$ & 7.4 \\
\hline VV Ser & $18: 26: 14.3$ & $+00: 06: 40$ & B9 & 440 & 0.54 & \multicolumn{3}{|c|}{16.9} & $3 \%$ & 7.7 \\
\hline MWC 300 & $18: 26: 45.0$ & $-06: 06: 48$ & $\mathrm{Be}$ & 15500 & 19 & \multicolumn{3}{|c|}{16.3} & $3 \%$ & -0.6 \\
\hline AS 310 & 18:30:41.7 & $-05: 00: 26$ & B0 & 2500 & 3.09 & \multicolumn{3}{|c|}{16.5} & $3 \%$ & 3.5 \\
\hline HD 200775 & 21:00:59.7 & $+67: 57: 56$ & B3 & 600 & 0.74 & \multicolumn{3}{|c|}{17.0} & $3 \%$ & 7.1 \\
\hline V645 Cyg & $21: 38: 10.6$ & $+50: 00: 43$ & $\mathrm{O} 7$ & 6000 & 7.42 & \multicolumn{3}{|c|}{17.1} & $3 \%$ & 2.2 \\
\hline $\mathrm{BD}+65^{\circ} 1637$ & 21:41:41.1 & $+65: 52: 49$ & B2 & 1000 & 1.23 & \multicolumn{3}{|c|}{17.0} & $3 \%$ & 6.0 \\
\hline $\mathrm{LkH} \alpha 257$ & $21: 52: 22.8$ & $+46: 57: 58$ & $\mathrm{~B} 8$ & 900 & 1.11 & \multicolumn{3}{|c|}{17.0} & $3 \%$ & 6.2 \\
\hline $\mathrm{LkH} \alpha 233$ & $22: 32: 28.3$ & $+40: 24: 32$ & A7 & 880 & 1.09 & \multicolumn{3}{|c|}{17.0} & $3 \%$ & 6.3 \\
\hline HD 216629 & $22: 51: 18.4$ & $+61: 52: 46$ & B2 & 725 & 0.90 & \multicolumn{3}{|c|}{17.0} & $3 \%$ & 6.7 \\
\hline
\end{tabular}

${ }^{a}$ Completness absolute magnitudes have been computed assuming the distance reported in Col. 5.

range of spectral types between $\mathrm{A} 7$ and $\mathrm{O} 9$, with only two stars (Z CMa, spectral type F5, and V645 Cyg, O7) outside of this interval.

The 26 new target stars are listed in Table 1 (data on the other 19 sources can be found in Tables 1 and 2 of Paper I). For each star we report in Col. 1 the name, in Cols. 2 and 3 the coordinates at the 1950 equinox, in Col. 4 the spectral type, in Col. 5 the distance from the Sun, in Col. 6 the projected physical size of the observed field at the assumed distance, in Cols. 7, 8 and 9 the limiting magnitudes in the $J, H$ and $K$ bands, in Col. 10 the photometric accuracy and in Col. 11 the completeness absolute magnitude $M_{K}^{c}$ (see discussion below).

In Fig. 2 the galactic positions of the observed sources (including the 19 listed in Paper I) are presented. There appears to be no stronger selection effect, with the exception that Ae stars tend to be closer to the Sun than Be stars, as expected.

\section{Observations and data reduction}

The observations of the first sample of 19 sources have been discussed in Paper I and will not be described again here. The new 26 stars have been observed during several runs at TIRGO (11 fields in $J, H$ and $K$ ) and at the Nordic Optical Telescope (NOT, 15 fields in $K$ only) using the Arcetri near infrared camera (ARNICA). ARNICA is equipped with a NICMOS3 $256 \times 256 \mathrm{HgCdTe}$ detector and a complete description of the instrument and its performances at TIRGO can be found in Lisi et al. (1996) and Hunt et al. (1996). The TIRGO observations were obtained between 1993 and 1996. The observing setup was the same as in Paper I, with a field of view of $\sim 7^{\prime} \times 7^{\prime}$ for all objects but $\mathrm{Z}$ CMa, for which we covered only an area of $\sim 4^{\prime} \times 4^{\prime}$. The ARNICA pixel scale at the TIRGO is $\sim 0.96^{\prime \prime}$, which well matches the typical seeing conditions $\left(2^{\prime \prime}-3^{\prime \prime}\right)$.

The NOT observations were carried out during a five night run from August $31^{\text {st }}$ to September $4^{\text {th }} 1996$. At NOT the ARNICA plate scale was $0.52^{\prime \prime} /$ pix in order to 

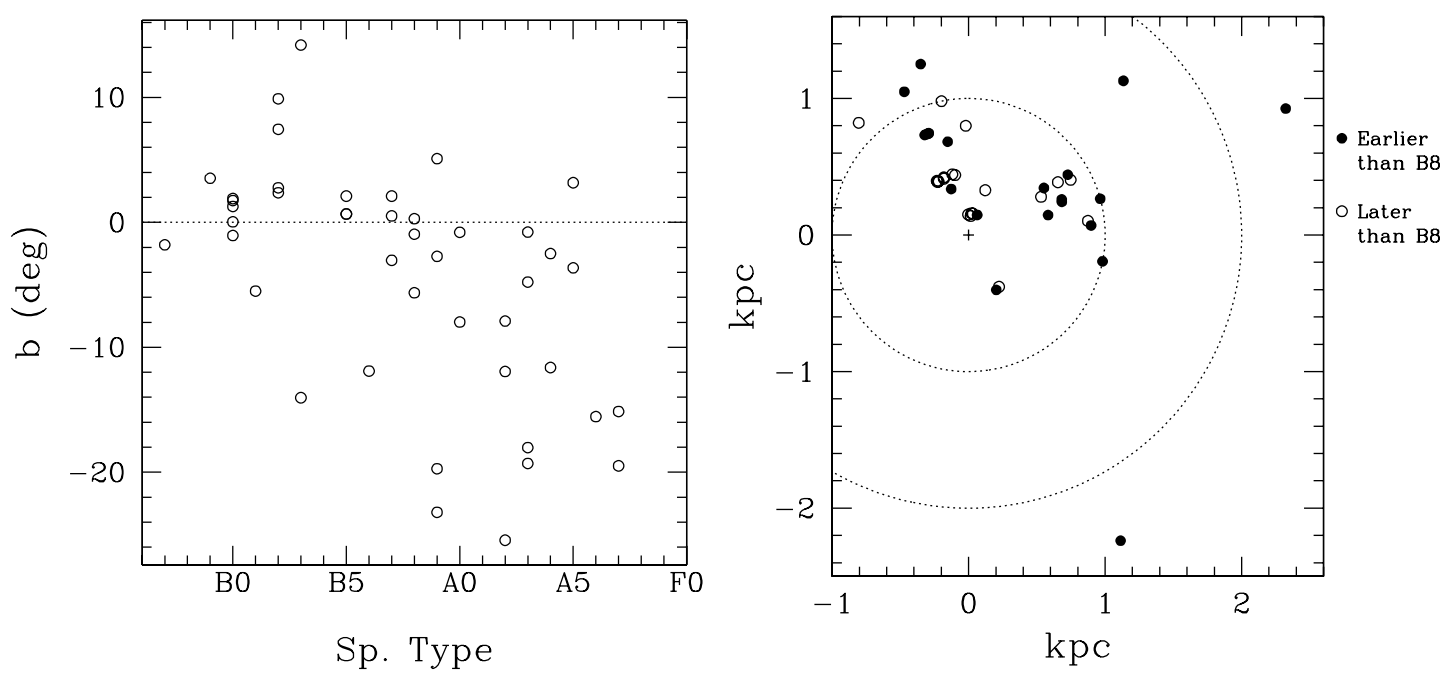

Fig. 2. Left: galactic latitude of the observed sources versus spectral type of the Herbig AeBe star. Right: position on the galactic plane of the observed stars; the position of the Sun is represented by a "+" and the Galactic Center is at $(0,-8.5)$, filled circles represent stars with spectral type earlier than B8, open circles stars later than B8. The two most distant stars (MWC 300 and V645 Cyg) are not included. The concentric circles are centered on the Sun position and have radii of 1 and $2 \mathrm{kpc}$

match the better seeing (in fact, during the whole run the images have been pixel-limited due to the excellent, subarcsecond, seeing conditions). The field of view of each image is thus $\sim 2^{\prime}$. In order to cover a larger field around each star, we used a mosaicing technique that yields a constant signal to noise ratio on a field of $\sim 8.5^{\prime}$ diameter centered on the target star; each mosaic consists of at least 42 partially overlapping frames. Flat fielding has been performed using differential flat frames constructed by subtracting one from the other two sky frames at different illumination, obtained by median averaging various sets of exposures at sunset and sunrise. The differential flat fielding was necessary due to the high, spatially non-uniform emissivity of the telescope (see also Hunt et al. 1996). After flat fielding, sky subtraction was performed on each frame using sky frame obtained by median averaging a set of exposures in the mosaic, as described in Hunt et al. (1994). After reduction the images were registered and combined to form the final large mosaics. All the data reduction has been performed using the IRAF ${ }^{1}$ and ARNICA (Hunt et al. 1994) software packages.

Photometric calibration was performed observing a set of near infrared photometric standard stars from the ARNICA (Hunt et al. 1998) list. The final calibration accuracy is $\sim 3 \%$ for all fields at the NOT and better than $8 \%$ at the TIRGO (except for $\mathrm{Z} \mathrm{CMa).} \mathrm{The} \mathrm{calibration}$ accuracy for each field is reported in Table 1.

As discussed in Paper I, the automatic star finding algorithms have not proven to be completely reliable in

\footnotetext{
1 IRAF is made available to the astronomical community by the National Optical Astronomy Observatories, which are operated by AURA, Inc., under contract with the U.S. National Science Foundation.
}

finding all (and only) the point sources in every field, primarily because of the bright diffuse emission associated with some of the Herbig AeBe stars. Source lists in all fields have thus been individually edited and corrected by inspecting the images at different contrast levels.

Aperture photometry on the detected point sources was performed using the IRAF DAOPHOT package and a 4 pixel aperture for both TIRGO and NOT observations, corresponding to $\sim 4^{\prime \prime}$ and $\sim 2^{\prime \prime}$ respectively. The $3 \sigma$ limiting magnitudes are reported in Table 1 . By checking the cumulative source count plots, we estimate our data to be complete down to one magnitude brighter than the limiting magnitude of each field. Note that, as in Paper I, the TIRGO limiting magnitudes refer to the "edges" of the mosaics. Since the fields have not been imaged with constant signal to noise, the central regions of the mosaics are $0.5-1$ magnitude deeper.

The completeness absolute magnitude in $K\left(M_{K}^{\mathrm{c}}\right)$ has been computed from the observed completeness magnitude assuming the distance reported in Table 1 (Col. 11). In computing $M_{K}^{c}$, we have neglected the effect of extinction. We note however that in the $K$ band the correction due to interstellar extinction is expected to be very low for all the sources, with the possible exception of the most distant ones.

\section{Results}

\subsection{Stellar parameters}

The properties of 45 observed stars are summarized in Table 2, which gives an identification number (Col. 1), source name (Col. 2) spectral type (Col. 3), distance 
Table 2. Star parameters and mass sensitivities

\begin{tabular}{|c|c|c|c|c|c|c|c|c|c|c|c|c|c|}
\hline N. & Star & type & $\begin{array}{c}D \\
(\mathrm{pc})\end{array}$ & $\begin{array}{c}V \\
(\mathrm{mag})\end{array}$ & $\begin{array}{l}B-V \\
(\mathrm{mag})\end{array}$ & $\begin{array}{c}\log (T) \\
(K)\end{array}$ & $\begin{array}{c}\log (L) \\
\left(L_{\odot}\right)\end{array}$ & $\begin{array}{c}\text { age } \\
\text { (Myr) }\end{array}$ & $\begin{array}{c}M_{0} \\
\left(M_{\odot}\right)\end{array}$ & $\begin{array}{c}M_{2} \\
\left(M_{\odot}\right)\end{array}$ & $\overline{\mathcal{N}_{K}}$ & $\overline{I_{\mathrm{C}}}$ & $\begin{array}{c}r_{\mathrm{c}} \\
(\mathrm{pc})\end{array}$ \\
\hline 1 & V645 Cyg & $\mathrm{O} 7$ & 6000 & 13.19 & 1.07 & 4.56 & 5.11 & - & - & - & $>5$ & $29.5 \pm 2$ & 0.6 \\
\hline 2 & MWC 297 & O9 & 450 & 12.11 & 2.14 & 4.52 & 4.60 & - & - & - & 37 & $20.4 \pm 1$ & 0.05 \\
\hline 3 & MWC 137 & B0 & 1300 & 11.93 & 1.30 & 4.49 & 4.48 & - & - & - & $>59$ & $76.0 \pm 9$ & 0.4 \\
\hline 4 & R Mon & B0 & 800 & 11.92 & 0.72 & 4.49 & 3.34 & - & - & - & 0 & $-12.8 \pm 3$ & - \\
\hline 5 & BHJ 71 & B0 & 730 & 10.25 & 0.83 & 4.49 & 4.06 & - & - & - & 4 & $4.0 \pm 3$ & 0.15 \\
\hline 6 & MWC 1080 & B0 & 2500 & 11.28 & 1.36 & 4.49 & 5.38 & - & - & - & $>9$ & $31.0 \pm 3$ & 0.7 \\
\hline 7 & AS 310 & B0 & 2500 & 12.39 & 1.05 & 4.49 & 4.55 & - & - & - & $>37$ & $70.0 \pm 17$ & 0.4 \\
\hline 8 & RNO 6 & B1 & 1600 & 14.52 & 0.57 & 4.35 & 2.34 & - & - & - & $>11$ & $11.0 \pm 1$ & 0.3 \\
\hline 9 & HD 52721 & B2 & 1150 & 6.38 & 0.05 & 4.31 & 4.53 & - & - & - & 10 & $20.5 \pm 4$ & 0.6 \\
\hline 10 & $\mathrm{BD}+65^{\circ} 163$ & B2 & 1000 & 10.05 & 0.42 & 4.31 & 3.40 & - & - & - & 29 & $75.0 \pm 5$ & 0.4 \\
\hline & HD 216629 & B2 & 725 & 9.25 & 0.75 & 4.31 & 3.85 & - & - & - & 29 & $34.0 \pm 6$ & 0.1 \\
\hline 12 & $\mathrm{BD}+40^{\circ} 4124$ & B2 & 1000 & 10.61 & 0.79 & 4.31 & 3.64 & - & - & - & 19 & $11.0 \pm 3$ & 0.2 \\
\hline 13 & HD 37490 & B3 & 360 & 4.50 & -0.08 & 4.25 & 3.95 & - & - & - & 9 & $9.9 \pm 3$ & 0.14 \\
\hline 14 & HD 200775 & B3 & 600 & 7.35 & 0.42 & 4.25 & 3.87 & - & - & - & 8 & $1.9 \pm 1$ & - \\
\hline 15 & MWC 300 & $\mathrm{Be}$ & 15500 & - & - & - & - & - & - & - & $>2$ & $21.0 \pm 8$ & 4 \\
\hline 16 & RNO 1B & $\mathrm{Be}$ & 850 & - & - & - & - & - & - & - & 12 & $9.7 \pm 1$ & 0.15 \\
\hline 17 & HD 259431 & B5 & 800 & 8.62 & 0.27 & 4.14 & 3.16 & 0.05 & $<0.1$ & $<0.1$ & 2 & $0.9 \pm 2$ & - \\
\hline 18 & XY Per & B6 & 160 & 8.99 & 0.50 & 4.11 & 1.83 & 2.0 & $<0.1$ & $<0.1$ & 3 & $11.3 \pm 3$ & 0.08 \\
\hline 19 & $\mathrm{LkH} \alpha 25$ & B7 & 800 & 12.60 & 1.18 & 4.09 & 2.56 & 0 . & $<0.1$ & 0.16 & 11 & $14.5 \pm 5$ & 0.3 \\
\hline 20 & HD 250550 & B7 & 700 & 9.47 & 0.07 & 4.09 & 2.32 & 0. & $<0.1$ & 0.18 & 4 & $2.2 \pm 2$ & - \\
\hline 21 & $\mathrm{LkH} \alpha 215$ & B7 & 800 & 10.18 & 0.53 & 4.09 & 2.72 & 0.1 & $<0.1$ & 0.13 & 7 & $3.9 \pm 1$ & 0.18 \\
\hline 22 & $\mathrm{LkH} \alpha 257$ & B8 & 900 & 13.18 & 0.78 & 4.05 & 1.85 & 2.0 & $<0.1$ & 0.29 & 15 & $5.5 \pm 6$ & - \\
\hline & $\mathrm{BD}+61^{\circ} 15$ & B8 & 650 & 10.25 & 0.59 & 4.05 & 2.51 & 0.1 & $<0.1$ & $<0$ & 8 & $-1.4 \pm 3$ & - \\
\hline 24 & VY Mon & B8 & 800 & 12.84 & 1. & 4.05 & 2.88 & 0.0 & $<0.1$ & $<0$ & 25 & $23.2 \pm 5$ & 0.25 \\
\hline 25 & VV Ser & B9 & 440 & 11.58 & 0.97 & 4.03 & 2.03 & 1 & $<0.1$ & $<0.1$ & 24 & $16.9 \pm 5$ & 0.1 \\
\hline 26 & V380 Ori & B9 & 460 & 10.30 & 0.56 & 4.03 & 2.07 & 1.0 & $<0.1$ & $<0.1$ & 3 & $-2.0 \pm 2$ & - \\
\hline 27 & V1012 Ori & B9 & 460 & - & - & - & - & - & - & - & 4 & $1.9 \pm 2$ & - \\
\hline & $\mathrm{LkH} \alpha 218$ & B9 & 1150 & 11.81 & 0.42 & 4.03 & 2.09 & 1. & $<0.1$ & 0.42 & 8 & $2.0 \pm 5$ & - \\
\hline 29 & $\mathrm{AB}$ & $\mathrm{Ac}$ & 160 & 6.96 & 0.13 & 3.99 & 1.86 & 1. & $<0.1$ & $<0.1$ & $>3$ & $3.0 \pm 6$ & - \\
\hline 30 & VX Cas & A 0 & 760 & 10.94 & 0.38 & 3.99 & 1.94 & 1 & $<0.1$ & 0.11 & 13 & $4.5 \pm 4$ & 0.3 \\
\hline & HD 2 & $\mathrm{~A} 2$ & 400 & 9.85 & 0.10 & 3.96 & 1.34 & 7.0 & $<0.1$ & 0.17 & 10 & $4.5 \pm 5$ & - \\
\hline & MWC 480 & $\mathrm{~A} 2$ & 140 & 7.65 & 0.16 & 3.96 & 1.34 & 7.0 & $<0.1$ & $<0.1$ & $>3$ & $5.0 \pm 6$ & - \\
\hline & UX Ori & A2 & 460 & 9.70 & 0.40 & 3.96 & 1.89 & 1.0 & $<0.1$ & $<0.1$ & 0 & $-0.3 \pm 1$ & - \\
\hline & T Ori & A & 460 & 10.15 & 0.58 & 3.94 & 1.8 & $1 .($ & $<0.1$ & 0.10 & 5 & $1.0 \pm 2$ & - \\
\hline 35 & IP Per & A3 & 350 & 10.35 & 0.41 & 3.94 & 1.36 & $7 .($ & $<0.1$ & 0.12 & 3 & $5.3 \pm 4$ & - \\
\hline 36 & $\mathrm{LkH} \alpha 2$ & A3 & 1000 & 11.52 & 0.48 & 3.94 & 1.89 & $1 .($ & $<0.1$ & 0.19 & 4 & $2.2 \pm 5$ & - \\
\hline & MWC 758 & A3 & 150 & 8.31 & 0.37 & 3.94 & 1.39 & 6.0 & $<0.1$ & $<0.1$ & $>2$ & $3.4 \pm 1$ & 0.03 \\
\hline & RR Tau & A4 & 800 & 10.10 & 0.60 & 3.93 & 2.37 & 0.1 & $<0.1$ & 0.12 & 7 & $0.8 \pm 6$ & - \\
\hline & HK Ori & A4 & 460 & 11.38 & 0.55 & 3.93 & 1.32 & 7.5 & 0.11 & 0.49 & 7 & $2.2 \pm 1$ & - \\
\hline 40 & $\mathrm{MaC} \mathrm{H} 12$ & A5 & 850 & - & - & - & - & - & - & - & 15 & $5.1 \pm 1$ & 0.15 \\
\hline & $\mathrm{LkH} \alpha 198$ & A 5 & 600 & 13.97 & 0.97 & 3.92 & 0.96 & 10.0 & 0.11 & 0.46 & 6 & $-10.6 \pm 11$ & - \\
\hline & Elias 1 & A6 & 160 & 15.3 & 1.5 & - & - & - & - & - & $>2$ & $2.0 \pm 3$ & - \\
\hline & BF Ori & $\mathrm{A} 7$ & 460 & 9.59 & 0.26 & 3.90 & 1.53 & 3.0 & $<0.1$ & 0.14 & 4 & $1.1 \pm 1$ & - \\
\hline & $\mathrm{LkH} \alpha 23$ & A7 & 880 & 12.93 & 0.84 & 3.90 & 1.48 & 4.0 & $<0.1$ & 0.39 & 2 & $1.0 \pm 1$ & - \\
\hline 45 & $\mathrm{Z} \mathrm{CMa}$ & F5 & 1150 & 8.74 & 1.29 & - & - & - & - & - & $>0$ & $-5.0 \pm 5$ & - \\
\hline
\end{tabular}

(Col. 4), $V$-magnitude (Col. 5), $(B-V)$ colour (Col. 6), effective temperature $T_{\text {eff }}$ (Col. 7), and luminosity of the star (Col. 8). In Col. 9 we report the calculated age of the Herbig AeBe star (see Sect. 4.2), while the minimum mass limits corresponding to 0 and 2 magnitude of extinction in $K$ are given in Cols. 10-11 (see Sect. 4.3). The stars are listed according to their spectral type, from the earliest to the latest. Spectral types, distances, $V$-magnitudes and $(B-V)$ colors are taken from the literature, as specified in the comments on individual stars. We determined the effective temperature $T_{\text {eff }}$ from the spectral type, using the scale of Cohen \& Kuhi (1979). The luminosity was then computed by fitting a blackbody of temperature $T_{\text {eff }}$ and varying radius to the de-reddened $V$-band magnitude and distance. In all cases, we have assumed a value of the total to selective extinction $R_{V}=3.1$. The bolometric 


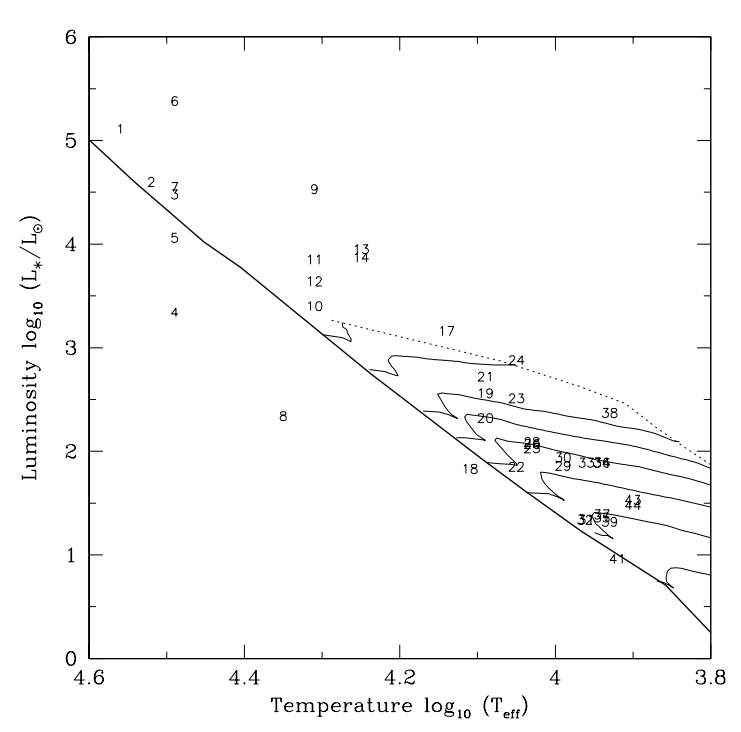

Fig. 3. Distribution of 39 Herbig AeBe stars in the $\mathrm{H}-\mathrm{R}$ diagram. Each star is labeled by its reference number as in Table 2. The solid lines are the evolutionary tracks for $M_{*}=1.5,2,2.5$, $3,3.5,4,5$ and $6 M_{\odot}$ (from bottom to top). The dotted line is the birthline of Palla \& Stahler (1990). The heavy solid line is the theoretical zero-age main-sequence

corrections are from Schmidt-Kaler (1981). For variable stars, we have used the $V$-magnitude in the brightest state, assuming that the variability is due to circumstellar extinction.

\subsection{Age estimates}

The distribution in the $\mathrm{H}-\mathrm{R}$ diagram of the 39 Herbig AeBe stars with a determination of temperature and luminosity is shown in Fig. 3. We have estimated the stellar ages from their location in the $\mathrm{H}-\mathrm{R}$ diagram using the evolutionary tracks and isochrones from Palla \& Stahler (1993). There is a clear distinction between Herbig AeBe stars of the earliest spectral types (O7 to B5) and those with types later than B5. For the former an age estimate based on the $\mathrm{H}-\mathrm{R}$ diagram is not possible since they lie either on or above the ZAMS and do not have an optically visible PMS phase. These stars include number 1 to 14 of Table 2. There are two stars, R Mon (No. 4) and RNO 6 (No. 8), which lie far below the ZAMS, in a forbidden part of the diagram. This may be to due difficulties in deriving the correct stellar photometry and reddening in regions of heavy nebular emission.

Herbig AeBe stars from B5 to A7 are well distributed at or below the birthline and an age estimate is thus possible. The individual ages (in million years) are listed in Table 2 and span a wide range from $0.1 \mathrm{Myr}$ for stars near the birthline to $10 \mathrm{Myr}$ for $\mathrm{LkH} \alpha$ 198, the oldest of the whole sample. Our age estimates reflect the procedure adopted to compute the stellar parameters and are subject to several sources of uncertainty. In general, we obtain values of the luminosity that are on the low side of those published in the literature, resulting in greater ages for the Herbig AeBe stars. In a few cases, the difference can be quite substantial, up to factors greater than 10. Examples include $\mathrm{LkH} \alpha 25, \mathrm{LkH} \alpha 198$ and $\mathrm{LkH} \alpha$ 233. A possible cause of the discrepancy in luminosity could be due to our assumption of a single value of $R_{V}$ for all the stars. In fact, the bolometric luminosity depends sensitively on $R_{V}$ and it is well known that many Herbig AeBe stars present anomalous extinction, suggesting higher values of $R_{V}$ than for the standard interstellar case. As an example, a variation of $R_{V}$ from 3.1 to 5.1 implies an increase of the luminosity of $\mathrm{LkH} \alpha 198$ from $\sim 10 L_{\odot}$ to $\sim 45 L_{\odot}$ with a corresponding decrease of age to a more realistic value of 1 Myr. Note that a larger value of $R_{V}$ may also move close to the ZAMS the two stars R Mon and RNO 6 .

In principle, a careful analysis of the appropriate value of $R_{V}$ could be done on each Herbig AeBe star, but this exercise goes beyond the purpose of this section in which the stellar ages are only used to obtain the mass sensitivity limits of our survey, as illustrated in the next subsection. Moreover, it is important to point out that the age estimates do not affect the determination of the clustering properties of Herbig AeBe stars.

\subsection{Limiting minimum mass}

Assuming that all the stars located around the Herbig AeBe star are coeval, it is possible to transform the $K$-completeness absolute magnitudes reported in Table 1 into an estimate of the lowest mass detectable in the field, the so-called minimum mass. For the transformation, we have used the method discussed in the Appendix (see also Meyer 1996), which allows one to derive at any given time a relation between the absolute $K$-magnitude and the mass of the star from a set of evolutionary tracks. For consistency, we should have used the same set of tracks employed for the age estimate. However, the models of Palla \& Stahler (1993) do not extend below 0.6 $M_{\odot}$, whereas the IR images are deep enough to probe masses near the brown dwarf limit. Thus, these tracks cannot be used for the determination of the minimum mass, and we have used instead the evolutionary tracks of D'Antona \& Mazzitelli (1994) which extend to substellar masses.

The minimum mass depends on the extinction at $K$ band. Even though the $K$ extinction is a factor $\sim 10$ (in magnitudes) less than in the visual and the extinction toward the Herbig AeBe star itself is usually $\leq 5$ mags in the visual, some of the colour-colour diagrams shown in the following section reveal that several stars in the fields are affected by a substantial amount of extinction ( $A_{V} \sim 10-20$ mags in some cases). We give in Table 2 the minimum mass in each field for $A_{K}=0$ and 2 mags 

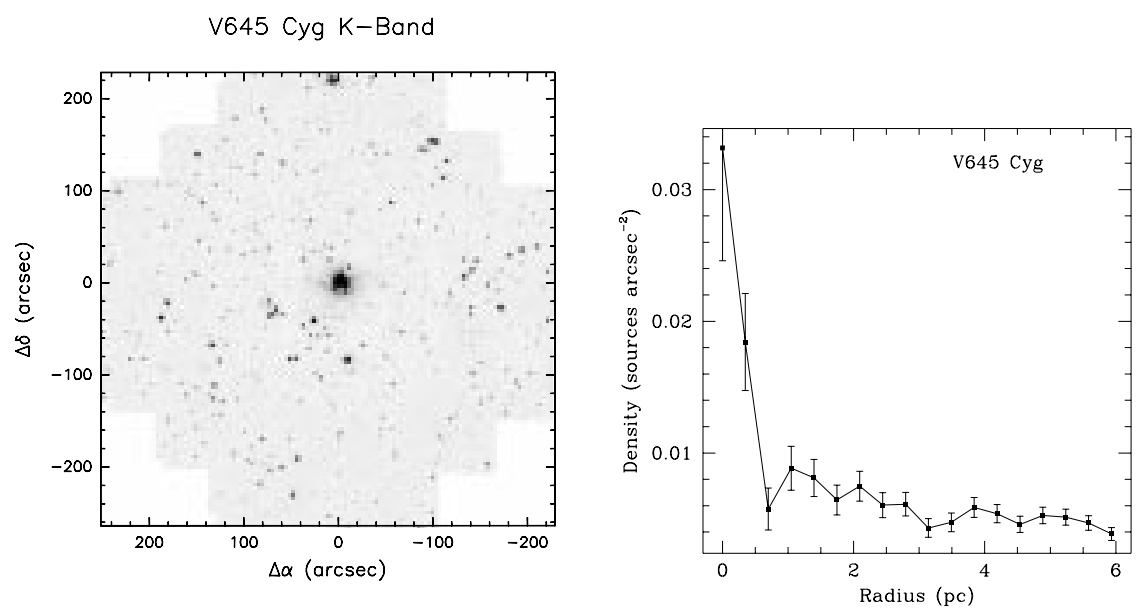

Fig. 4. V 645 Cyg. Left: $K$-band image; right: $K$-band source surface density profile
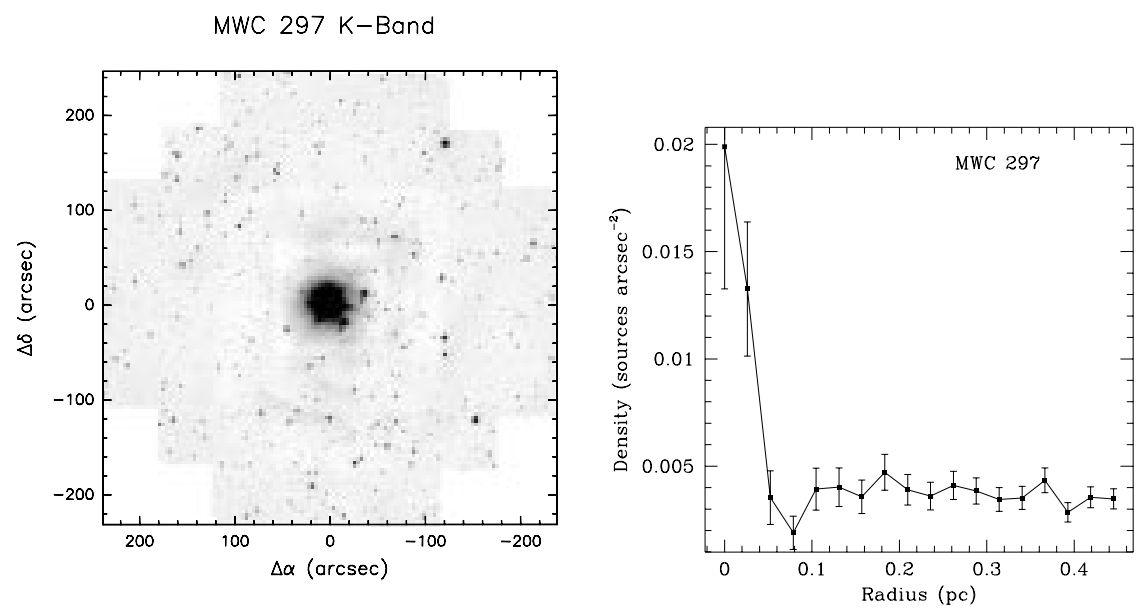

Fig. 5. MWC 297. Left: $K$-band image; right: $K$-band source surface density profile

(Cols. 10 and 11). Clearly, the calculation of the minimum mass is possible only for those fields where an age estimate of the Herbig AeBe star exists. A value $<0.1 M_{\odot}$ in Table 2 means that the mass limit is smaller than the minimum mass available from the PMS evolutionary tracks of D'Antona \& Mazzitelli (1994).

Note that the derivation of the minimum mass assumes that all the observed emission at $K$-band is due to the stellar photosphere (i.e. the infrared excess is not considered and corrected for).

\subsection{Cluster indicators}

Several methods of measuring the richness of an embedded cluster of stars associated to the visible Herbig AeBe stars have been discussed in Paper I. We compute for each of the observed fields two such indexes, namely $\mathcal{N}_{K}$ and $I_{\mathrm{C}}$ which are given in Table 2, Cols. 12 and 13.

$\mathcal{N}_{K}$ is defined as the number of stars detected in the $K$-band image of the field within a distance of $0.21 \mathrm{pc}$ from the Herbig AeBe star with an absolute magnitude $M_{K}<$ $5.2 \mathrm{mag}$. The first of these two constraints is set to match the size of the best-studied clusters (e.g., BD+40 4124 : Hillenbrand et al. 1995; Palla et al. 1995). The threshold at $M_{K}=5.2 \mathrm{mag}$ is low enough to include at least some stars in most of the fields. In fact, there are 5 fields (those centered on Elias 1, MWC 758, MWC 480, AB Aur and $\mathrm{XY}$ Per) which have been imaged with a field of view smaller than $0.21 \mathrm{pc}$, and 7 (centred on Z CMa, MWC 300, AS 310, MWC 1080, V 645 Cyg, RNO 6 and MWC 137) which have $M_{K}^{\mathrm{c}}<5.2 \mathrm{mag}$. The values of $\mathcal{N}_{K}$ in Table 2 for these 12 objects should be considered as lower limits. Note that $\mathcal{N}_{K}$ can be directly compared to the results in Hillenbrand (1995).

The second, more reliable richness indicators is the quantity $I_{\mathrm{C}}$, which corrects for the "local" background/foreground contamination. $I_{\mathrm{C}}$ is computed by integrating the source surface density profile centered on the Herbig AeBe star $n(r)$ and by subtracting the average surface density measured at the edge of each field $n_{\infty}$ :

$I_{\mathrm{C}}=\sum_{i=0}^{i_{\max }} \pi\left(n\left(r_{i}\right)-n_{\infty}\right)\left(r_{i+1}^{2}-r_{i}^{2}\right)$ 


\section{MWC 137}
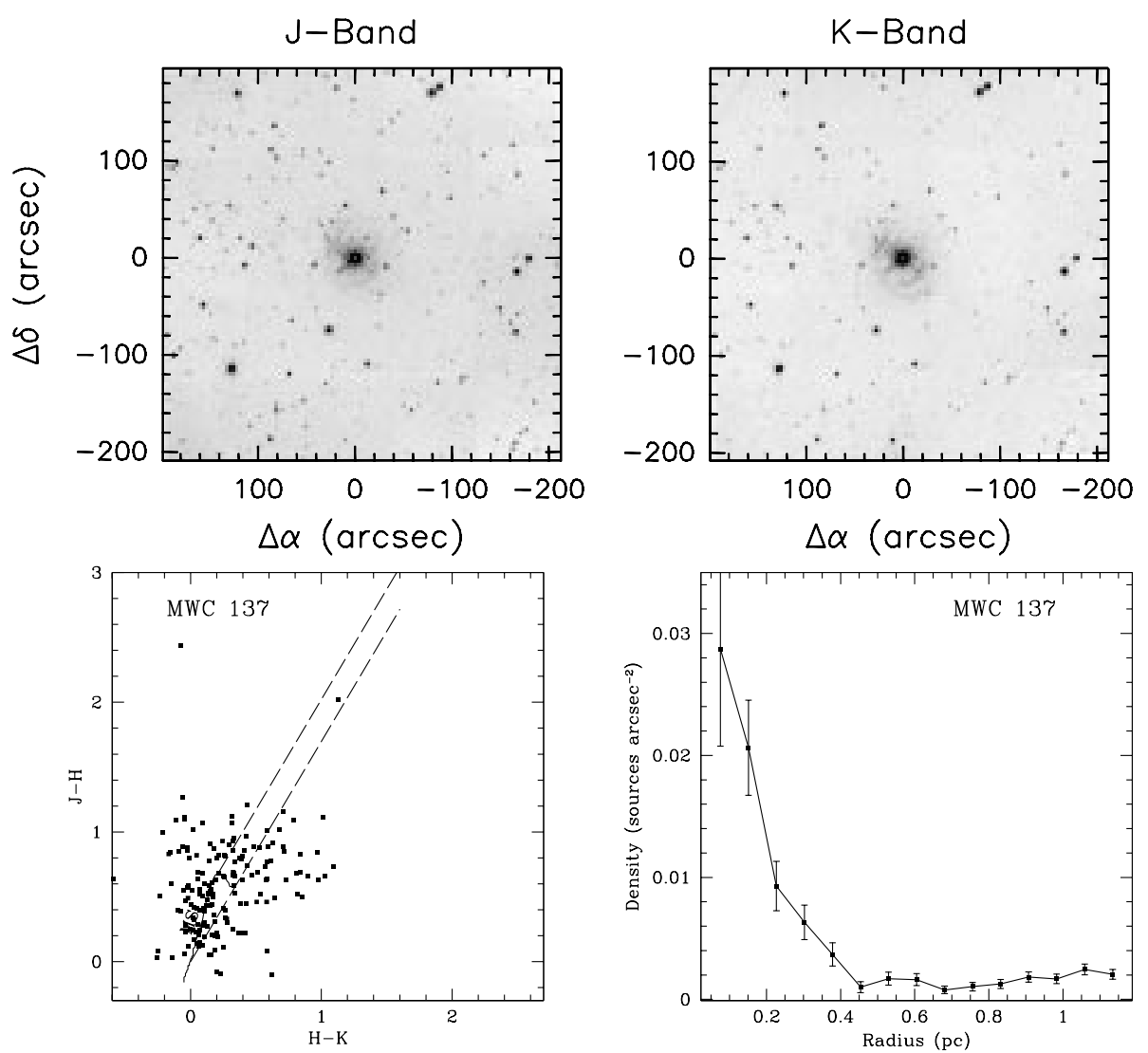

Fig. 6. MWC 137. Top left: $J$-band image; top right: $K$-band image; bottom left: colour-colour diagram; bottom right: $K$-band source surface density profile

where $n\left(r_{i}\right)$ is the local source surface density (i.e. the number of stars between $r_{i}$ and $r_{i+1}$ arcsec from the Herbig AeBe star divided by the area $\left.\pi\left(r_{i+1}^{2}-r_{i}^{2}\right)\right) \cdot i_{\max }$ is chosen to contain all the members of the cluster and $n_{\infty}$ is the mean value of $n\left(r_{i}\right)$ in the outer parts of the plot. In all fields $\Delta r \equiv r_{i+1}-r_{i}=12^{\prime \prime}$, which ensures a good "resolution" and (in most cases) a reasonable number of stars per annulus. The uncertainty on $I_{\mathrm{C}}$ has been computed by propagating the error in the determination of $n_{\infty}$ on the sum defined in Eq. (1).

The two indexes $I_{\mathrm{C}}$ and $\mathcal{N}_{K}$ suffer from different biases. In the case of $I_{\mathrm{C}}$, the main source of errors in comparing values for different fields arises from the fact that all sources detected within the completeness limit $M_{K}^{\mathrm{c}}$ have been included, in spite of the fact that $M_{K}^{c}$ varies from field to field. A second error derives from possible local extinction variations within the cluster (note that since $n_{\infty}$ is derived from observations of the same field, line-ofsight extinction does not affect $I_{\mathrm{C}}$ ). This effect leads to a systematic underestimate of the number of cluster members when a compact (with size of the order of the cluster size), high column density molecular clump is localized at the position of the Herbig AeBe star. In the case of $\mathcal{N}_{K}$, as we have already pointed out, the main uncertainty derives from background/foreground contamination. These points have been extensively examined in Paper I, and will not be discussed any further in here.

Both $I_{\mathrm{C}}$ and $\mathcal{N}_{K}$ are affected by the presence of bright reflection nebulosities associated to the Herbig AeBe star, which may "hide" low-luminosity companion stars. It is difficult to quantify this effect. We suggested in Paper I that it could provide an explanation for the very negative values of $I_{\mathrm{C}}$ in $\mathrm{LkH} \alpha 198$ and $\mathrm{R}$ Mon. Among the new fields observed in this paper, it certainly affects to an unknown degree the star counts in V645 Cyg.

In the last column of Table 2 we report an estimate of the stellar group radius, $r_{\mathrm{c}}$, for the fields in which a source density enhancement is detectable around the Herbig Ae/Be star. This typical size has been derived as the radius at which the sources density peak reachs the background level in the $K$-band sources surface density profiles presented below. 


\section{R Mon}
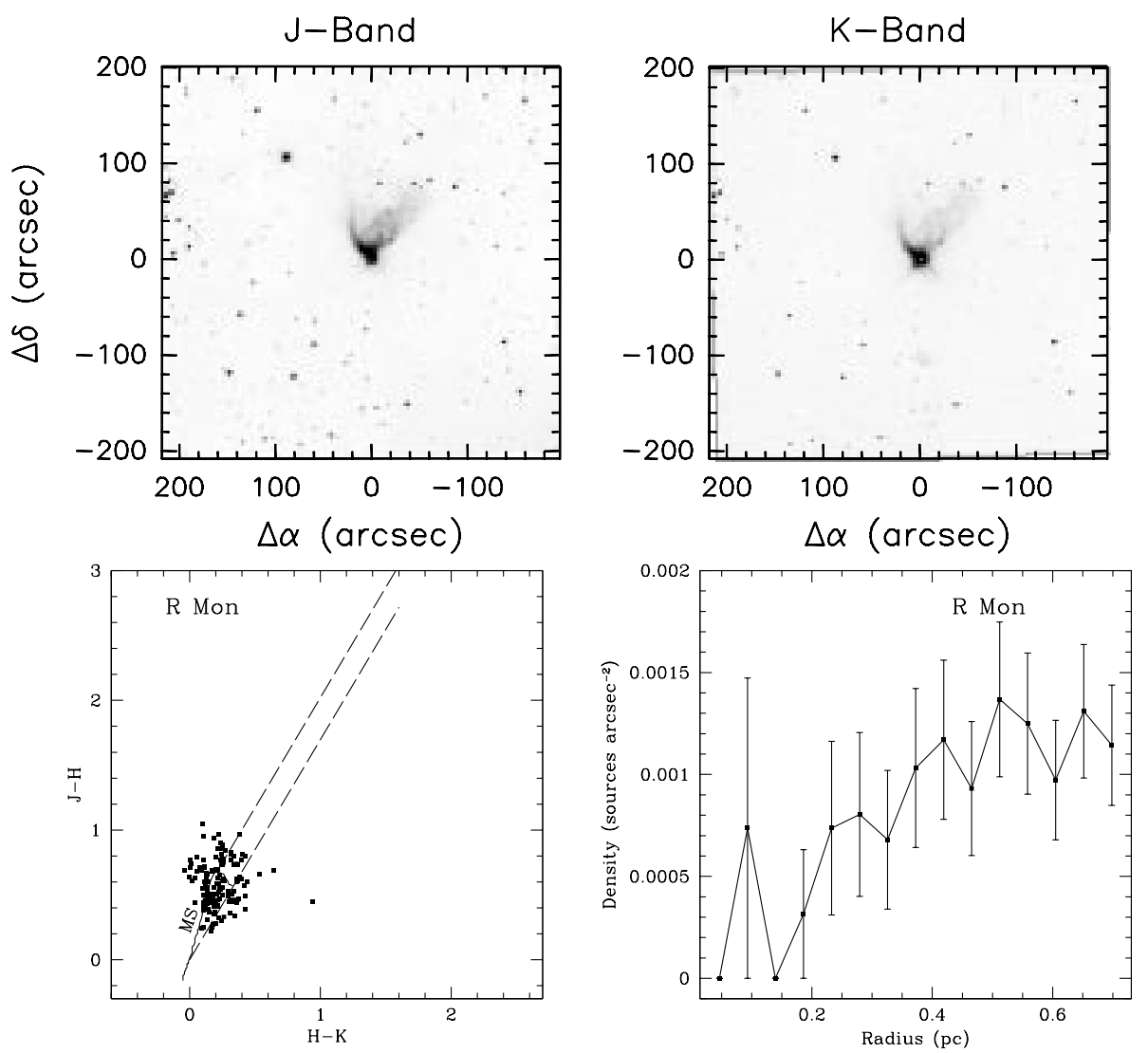

Fig. 7. R Mon. Top left: $J$-band image; top right: $K$-band image; bottom left: colour-colour diagram; bottom right: $K$-band source surface density profile

\section{Comments on individual sources}

In the following, we present a brief description of the basic stellar data for each Herbig AeBe star and of the associated stellar field. Where available, we also give some information about the amount of gas and dust associated with the stars, since this information will prove useful for the discussion of the clustering properties of the Herbig AeBe stars (see Testi et al. 1998). We have recomputed the mass of circumstellar dust and gas (in units of $M_{\odot}$ ) from the $1.3 \mathrm{~mm}$ flux as $1.2810^{-4}(D / 140 \mathrm{pc})^{2} F_{1.3 \mathrm{~mm}}(\mathrm{mJy})$ (Natta et al. 1997). Often there are large discrepancies between the circumstellar masses inferred from millimetric continuum observations and those derived from molecular data. Whenever possible, we give both of them. As in Table 2, the sources are ordered by decreasing spectral type.

For each Herbig AeBe system observed at TIRGO, we show the $J$ - and $K$-band images, the $(J-H, H-K)$ colour-colour diagram, and the stellar surface density profile at $K$-band. We determine $\mathcal{N}_{K}$ and $I_{\mathrm{C}}$ and provide an estimate of the radius of the stellar cluster, when a stellar density enhancement is present above the background. We have included the TIRGO observations of the 19 fields analysed in Paper I since only few fields were shown there. The 1996 NOT observations are limited to the $K$-band and therefore we show the $K$-band image and the corresponding surface density profile only. White pixels on top of the brighter stars indicate that these sources are saturated (or fall in the nonlinear regime of the detector).

For most of the sources observed in the earliest runs at the TIRGO, the scatter of the points in the colour-colour diagram is due either to the systematic uncertainties described in Paper I and in Hunt et al. (1996) or to the unstable atmospheric conditions which appear to plague more heavily the $H$-band data. In a few cases (HK Ori, HD 259431, LkH $\alpha 218, \mathrm{HD} 245185$, and Z CMa) a systematic offset in the $H$-band of $\sim 0.2-0.3$ magnitudes is evident.

\subsection{645 Cyg (AFGL 2798)}

The $V$-magnitude and $(B-V)$ colour are from Shevchenko et al. (1993). The distance (6 kpc) and the spectral type are from Cohen (1977). There is some controversy on the actual values of the parameters for this star, which 


\section{BHJ 71}
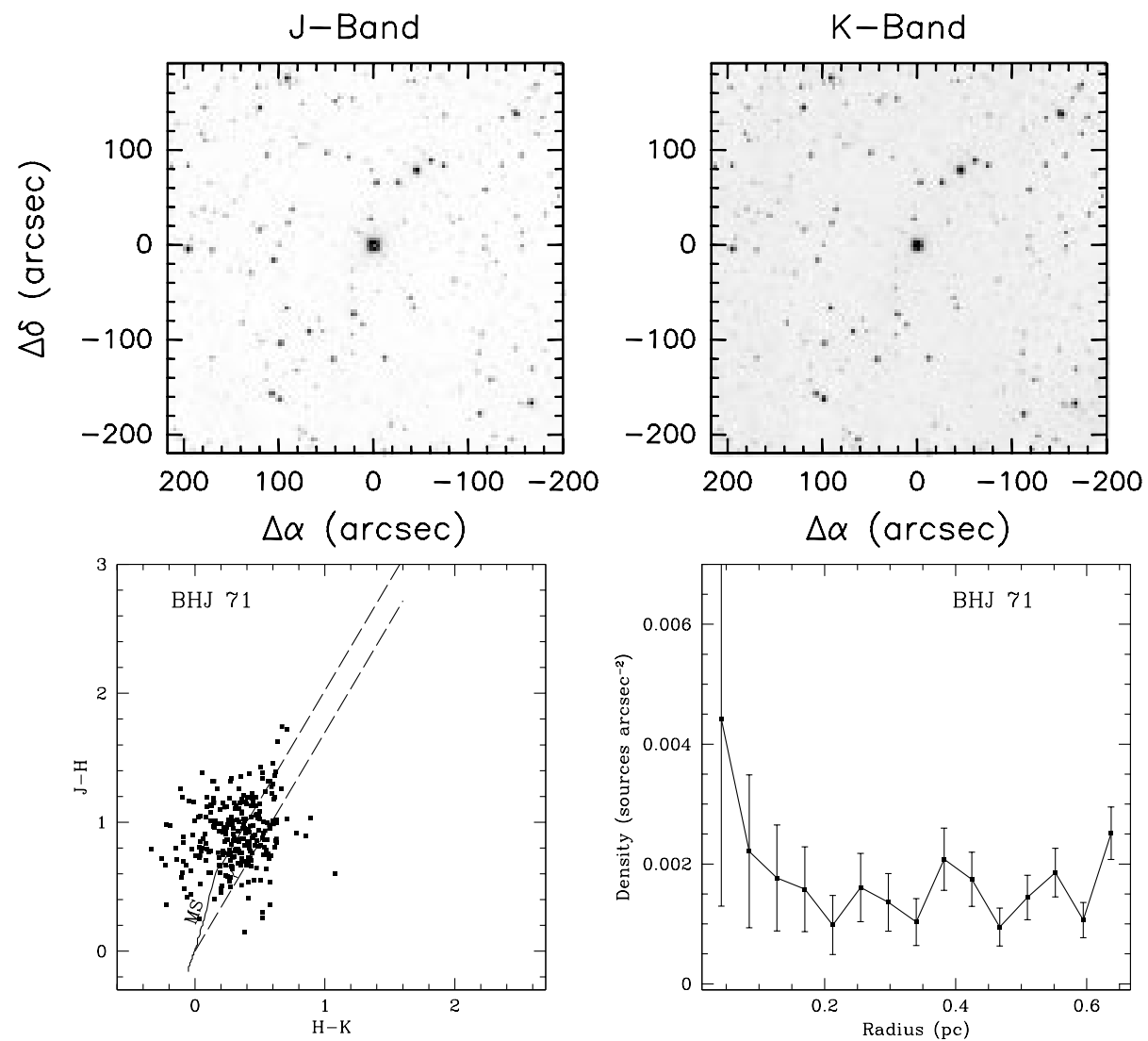

Fig. 8. BHJ 71. Top left: $J$-band image; top right: $K$-band image; bottom left: colour-colour diagram; bottom right: $K$-band source surface density profile

some authors (Goodrich 1986; Hamann \& Persson 1992; Corcoran \& Ray 1998) classify as A0 at distance 3500 pc.

Far-infared studies of this region (Natta et al. 1993) reveal extended emission at 50 and $100 \mu \mathrm{m}$, typical of an extended envelope surrounding the star. The estimated total mass of the envelope (within $0.5 \mathrm{pc}$ from the star) is $78 M_{\odot}$. Di Francesco et al. (1997) refer of a possible detection at $2.7 \mathrm{~mm}$ with the Plateau de Bure interferometer. In the radio continuum $(3.6 \mathrm{~cm}$; Skinner et al. 1993) the emission is extended, with an intensity consistent with the O7 classification of Cohen (1977).

$\mathrm{V} 645$ Cyg is located in a region of extended CO and $\mathrm{NH}_{3}$ emission (Torrelles et al. 1983, 1989). Torrelles et al. (1987) and Verdes-Montenegro et al. (1991) detected a massive $\mathrm{CO}$ outflow associated with the star.

(Fig. 4). The star is embedded in a bright diffuse nebula (see also Goodrich 1986). We detect a group of stars with a projected radius of $\sim 0.6 \mathrm{pc}$.

\section{2. $M W C 297 \quad(S S 73164)$}

The $V$-magnitude and $(B-V)$ colour are from Shevchenko et al. (1993). The distance $(450 \mathrm{pc})$ and the spectral type (O9) from Hillenbrand et al. (1992).

Hillenbrand et al. (1992) measured a $1.3 \mathrm{~mm}$ flux of $110 \mathrm{mJy}$ in a region of $0.06 \mathrm{pc}$ size, which roughly corresponds to a circumstellar mass of $0.15 M_{\odot}$. Mannings (1994) detected the star at all submm wavelengths; the SED is consistent with simple disc emission. However, Di Francesco et al. (1994) spatially resolve the source, implying contribution from an extended envelope. The molecular survey of Hillenbrand (1995) yields a mass estimate of $M_{\mathrm{cl}}=$ $1500 M_{\odot}$ in a region of $\sim 1 \mathrm{pc}$ in size.

(Fig. 5). Very bright star surrounded by a diffuse nebula. A stellar group is detected with a radius $r \sim 0.05 \mathrm{pc}$.

\section{3. $M W C 137 \quad(P K 195-00.1 ; P N V V$ 42)}

The $V$-magnitude and $(B-V)$ colour are from Shevchenko et al. (1993). The distance (1300 pc) and spectral type (B0) are from Hillenbrand et al. (1992). There is a big 

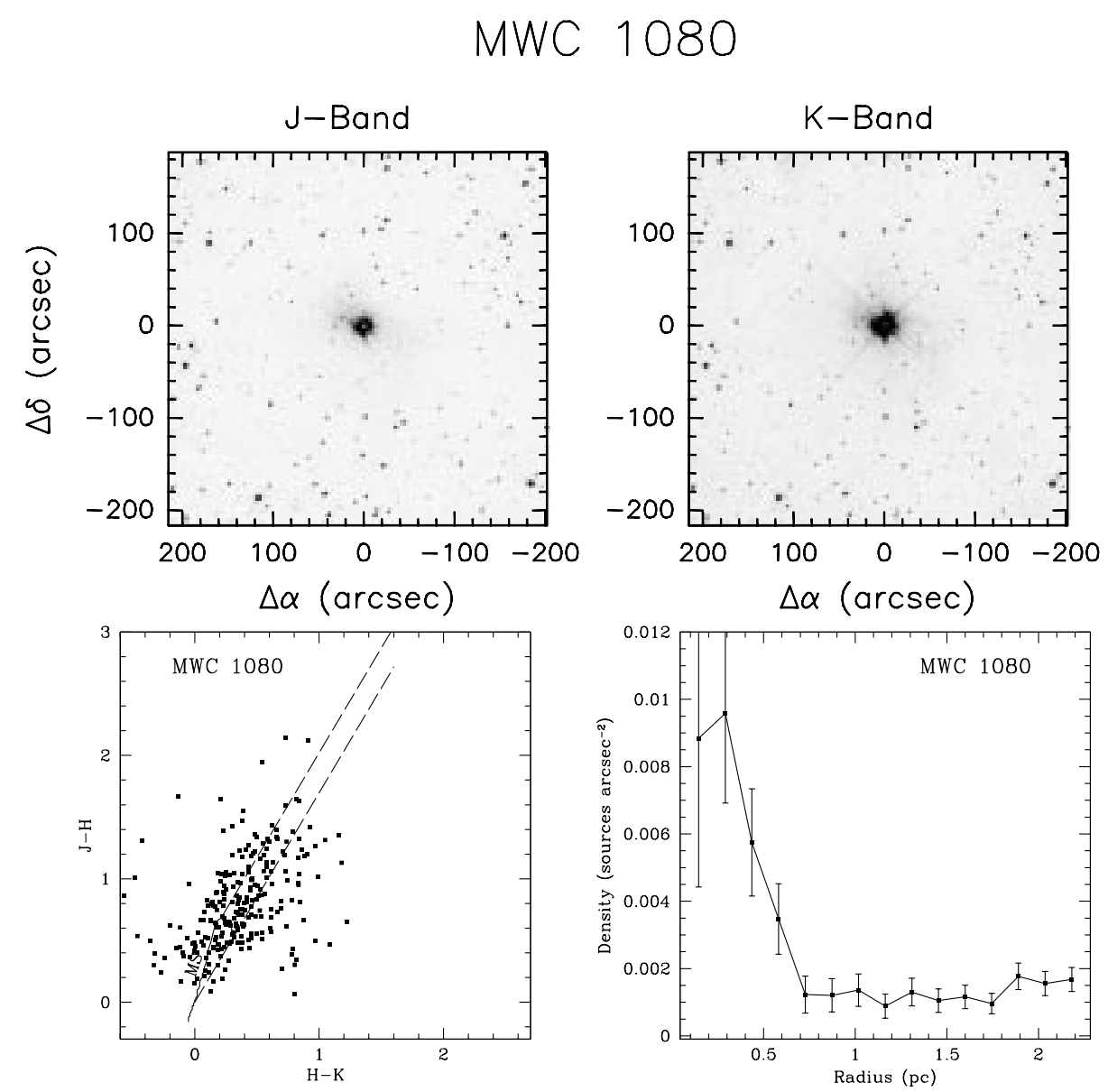

Fig. 9. MWC 1080. Top left: $J$-band image; top right: $K$-band image; bottom left: colour-colour diagram; bottom right: $K$-band source surface density profile
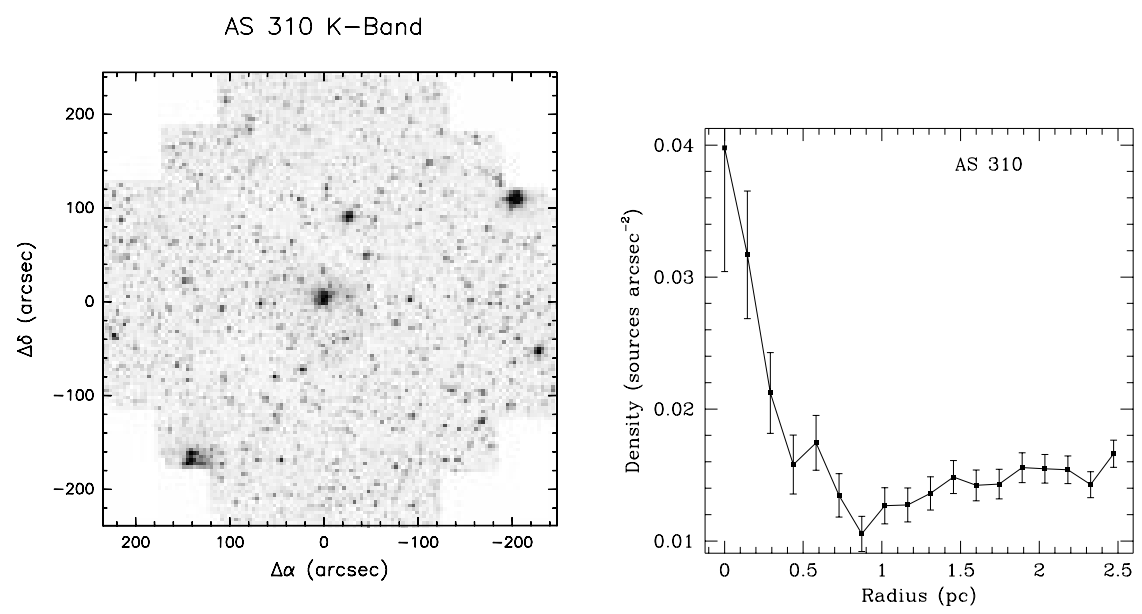

Fig. 10. AS 310. Left: $K$-band image; right: $K$-band source surface density profile 


\section{RNO 6}
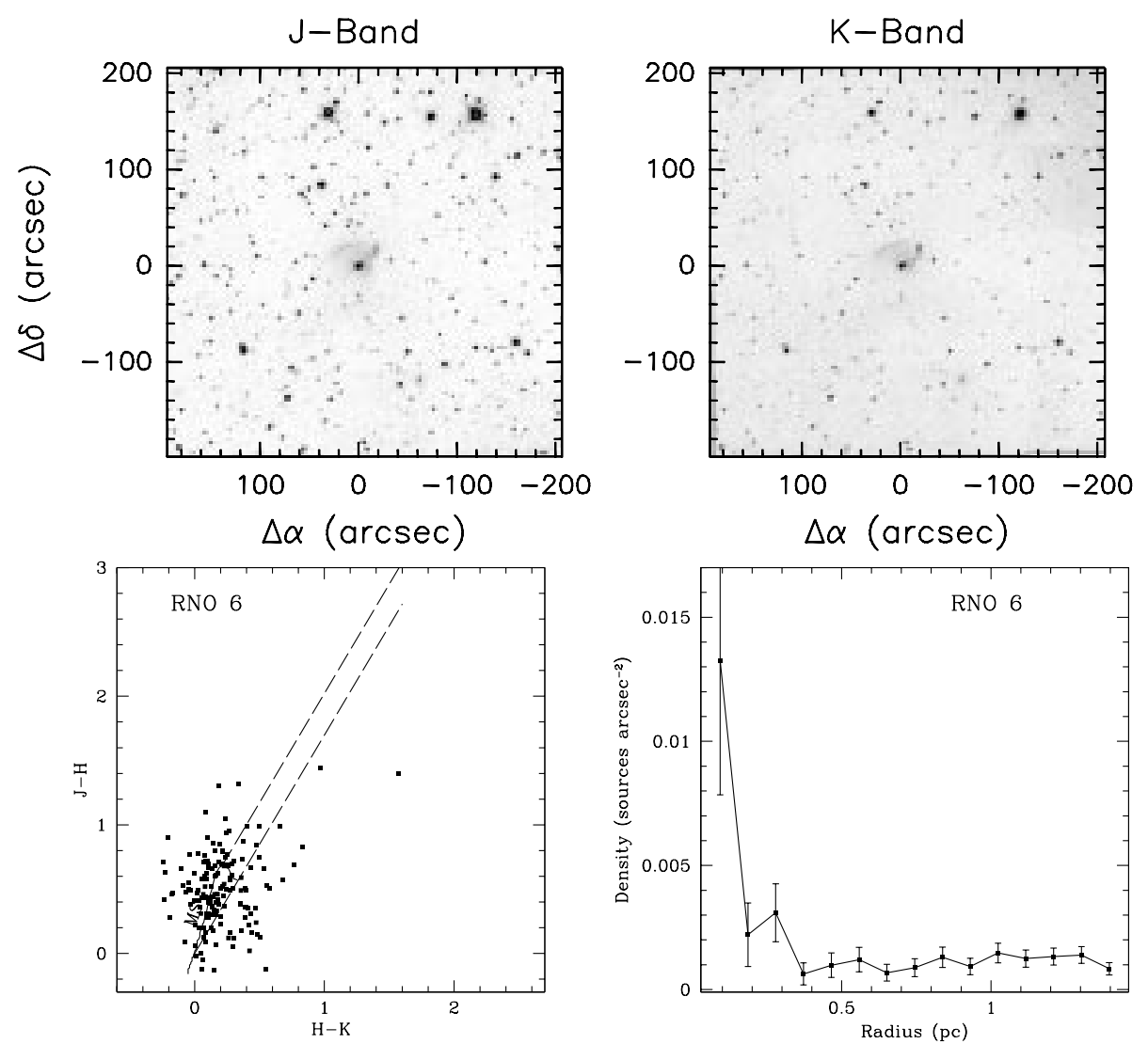

Fig. 11. RNO 6. Top left: $J$-band image; top right: $K$-band image; bottom left: colour-colour diagram; bottom right: $K$-band source surface density profile

discrepancy with Berrilli et al. (1992) parameters, but the latter seem rather uncertain.

The star was once believed to be a planetary nebula (Perek \& Kohoutek 1967; Acker et al. 1987; Acker \& Stenholm 1990) and its true nature is still disputed. However, Zijlstra et al. (1990) put it in the list of misclassified planetary nebulae from VLA data: it is shown to be a compact HII region based on radio morphology, stronger emission than most Planetary Nebulae and from its location in the IRAS colour-colour diagram.

The far-infrared (FIR) and (sub)mm environment of this star has been studied extensively in recent years. Di Francesco et al. (1994) found that the emission at 50 and $100 \mu \mathrm{m}$ peaks on the star but is quite extended. Standard accretion disks cannot be the sole source of FIR emission and most of the emission comes from an extended circumstellar envelope. The same conclusion is reached through submm observations by Mannings (1994), who found the emission at 350 and $450 \mu \mathrm{m}$ to be well in excess of that expected from an optically thick disk. The star has a $1.3 \mathrm{~mm}$ flux of $190 \mathrm{mJy}$ (Hillenbrand et al. 1992), which corresponds to a mass of circumstellar material of about $2 M_{\odot}$ in a region of 0.18 pc size. Di Francesco et al. (1997) possibly detect the source at $2.7 \mathrm{~mm}$ with the $\mathrm{PdB}$ interferometer giving further support to the hypothesys of the young nature of the object.

The molecular survey of Hillenbrand (1995) yields a mass estimate of $M_{\mathrm{cl}}=83 M_{\odot}$ in a region of $<0.8 \mathrm{pc}$ size. Higher resolution observations by Fuente et al. (1998) show that on a scale of $0.08 \mathrm{pc}$ the amount of gas and dust is reduced to $\sim 2 M_{\odot}$.

(Fig. 6). The Herbig AeBe star is surrounded by a double shell like reflection nebulosity. Within the nebula many faint stars are detected. The source surface density profile clearly shows a peak around MWC 137 with radius $r \sim 0.4 \mathrm{pc}$.

\subsection{R Mon $\left(B D+08^{\circ} 1427 ; M W C 151\right)$}

The $V$-magnitude and $(B-V)$ colour are from Shevchenko et al. (1993). These estimates may be affected by the bright nebulosity associated to the star. The distance (800 pc) is from Finkenzeller \& Mundt (1984) and the spectral type (B0) from Cohen \& Kuhi (1979).

Near infrared adaptive optics and visible HST imaging (Close et al. 1997) reveal a faint companion and resolve 


\section{HD 52721}
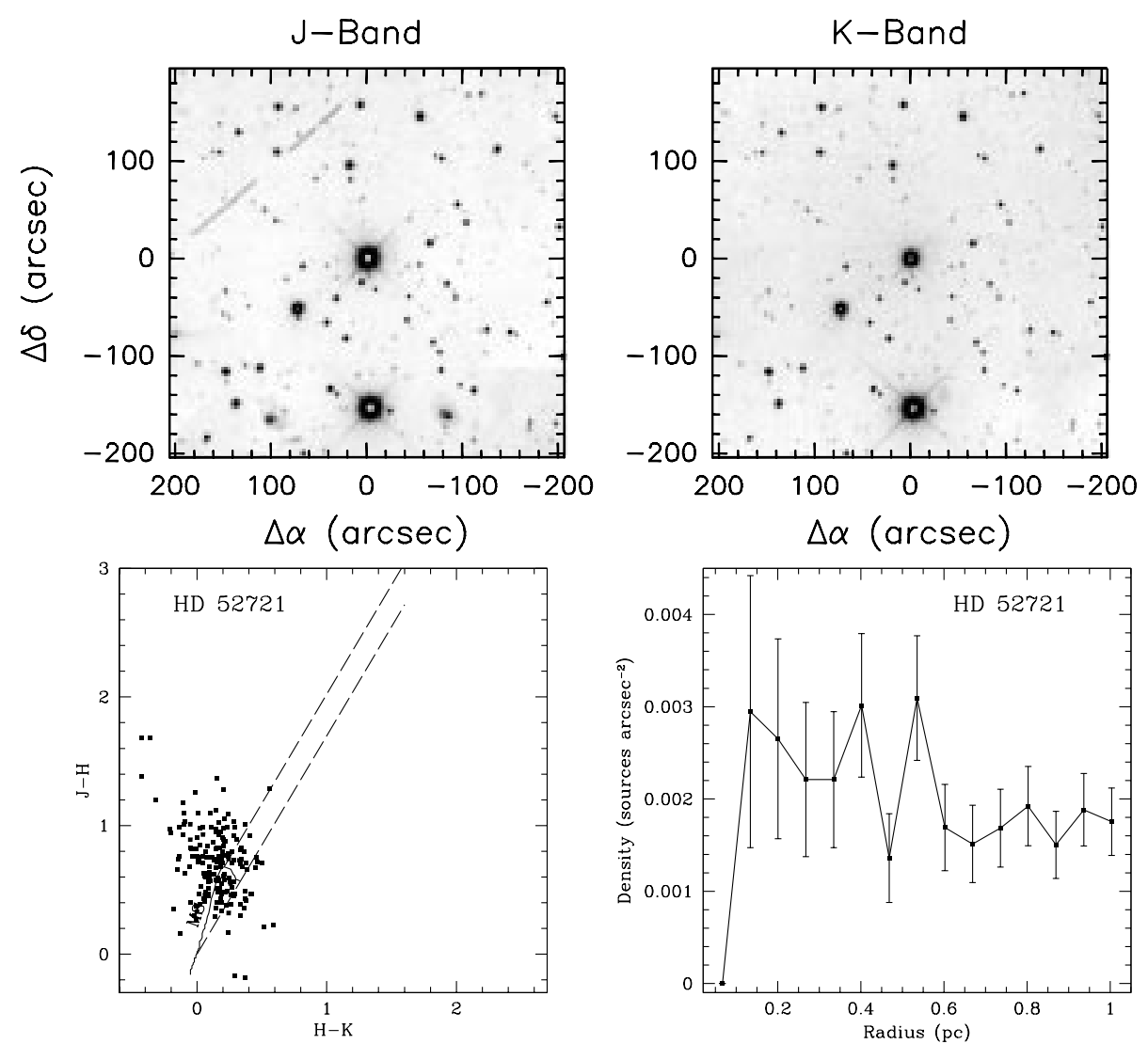

Fig. 12. HD 52721. Top left: $J$-band image; top right: $K$-band image; bottom left: colour-colour diagram; bottom right: $K$-band source surface density profile

the double helix structure of the diffuse nebulosity. No other object is found close to the Herbig Be star.

Natta et al. (1993) resolve the $100 \mu \mathrm{m}$ emission associated to the star and determine an envelope mass $>36 M_{\odot}$ (within a radius of $0.5 \mathrm{pc}$ ). The $1.3 \mathrm{~mm}$ flux (Hillenbrand et al. 1992) yields a value of the circumstellar mass in a region of $0.1 \mathrm{pc}$ size of about $0.4 M_{\odot}$.

(Fig. 7). The Herbig AeBe star is at the apex of an extended bright nebulosity. The source density profile shows a dip around the star position suggesting that background stars and possibly faint young companions might be hidden either by the bright nebula or a molecular clump.

\subsection{BHJ 71 (V374 Cep, AS 505)}

The $V$-magnitude and $(B-V)$ colour are from Shevchenko et al. (1993). The distance $(730 \mathrm{pc})$ is from Berrilli et al. (1992) and the spectral type (B0) from Finkenzeller $\&$ Mundt (1984).

Both the distance and the spectral classification are very uncertain. The distance quoted by Berrilli et al. (1992) is derived assuming that it is a member of the Cepheus OB3 association.
(Fig. 8). The field is very crowded, a moderate density enhancement with $r \sim 0.15 \mathrm{pc}$ is marginally detected.

\section{6. $M W C 1080$}

The $V$-magnitude and $(B-V)$ colour are from Shevchenko et al. (1993). The distance $(2500$ pc) is from Cantó et al. (1984) and the spectral type (B0) from Cohen \& Kuhi (1979).

The uncertainty on the stellar parameters is not large in this case. Agreement exists in the literature about the fact that the star is indeed young and a bona fide Herbig AeBe star. This is supported, among other things by the detection of a molecular outflow (Levreault 1988) and high velocity CO line emission (Koo 1989; Wu et al. 1996). Zinnecker \& Preibisch (1994) have detected strong X-ray emission from the object. Leinert et al. (1997) classify the star as an eclipsing binary and detect an infrared companion at $0.75^{\prime \prime}$. The companion appears to have a high luminosity and could possibly be a second Herbig AeBe star in the region. This result is confirmed by Pirzkal et al. (1997). These findings support the colliding winds hypothesis for the X-ray emission. 

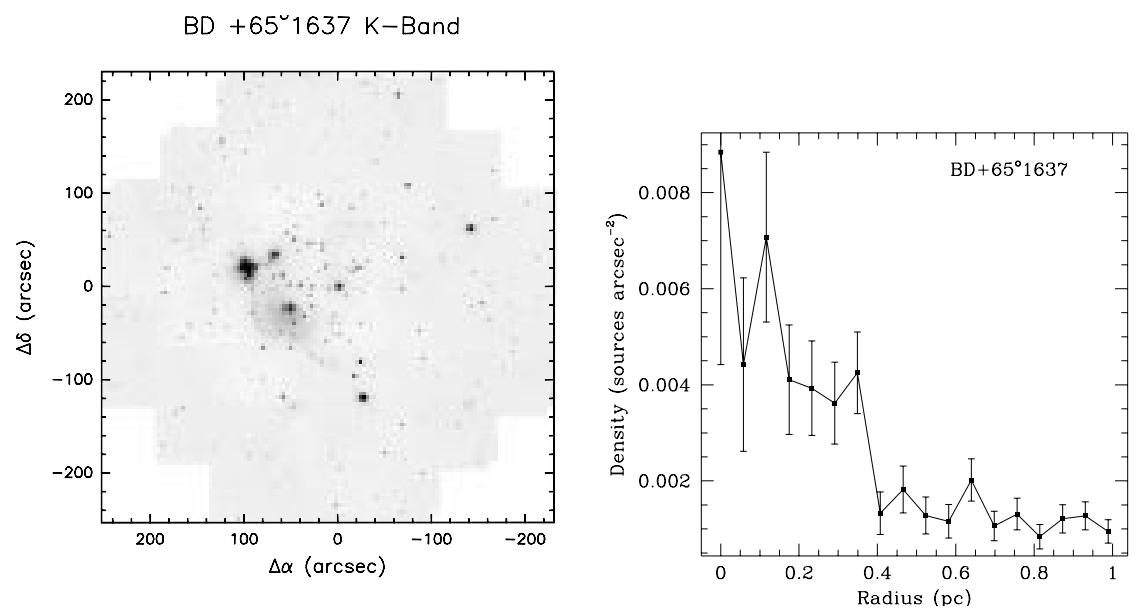

Fig. 13. $\mathbf{B D}+65^{\circ} 1637$. Left: $K$-band image; right: $K$-band source surface density profile
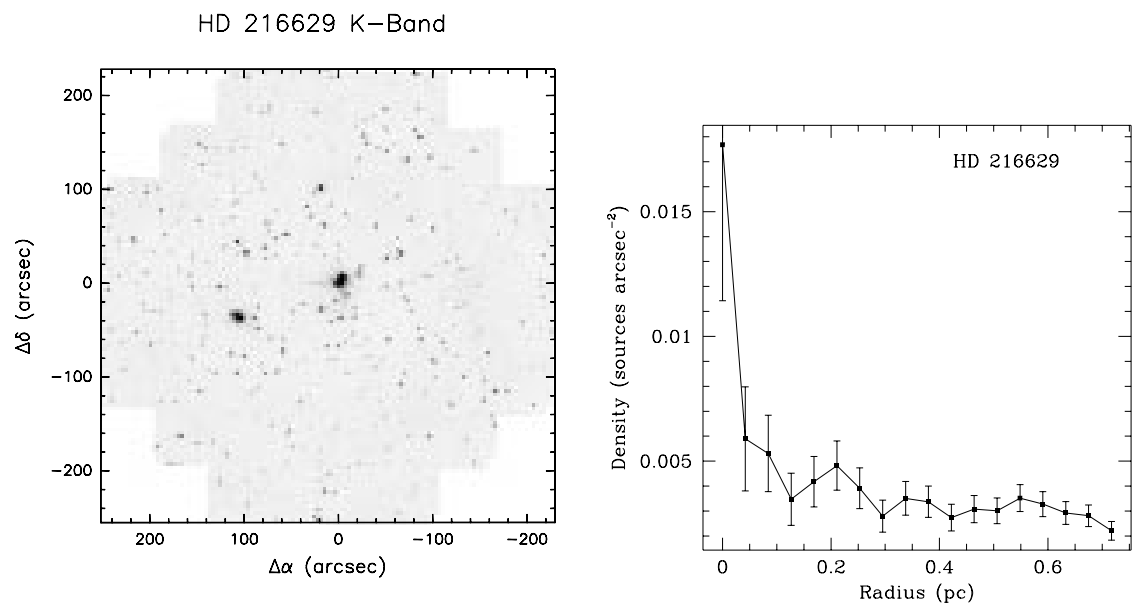

Fig. 14. HD 216629. Left: $K$-band image; right: $K$-band source surface density profile

The star is detected at $100 \mu \mathrm{m}$ by di Francesco et al. (1994), who derive an apparent size of $\sim 29^{\prime \prime}$ (corresponding to $\sim 310^{4} \mathrm{AU}$ ). The presence of an extended component is also inferred from the submm observations of Mannings (1994), who measures 350 and $450 \mu \mathrm{m}$ flux densities in excess to the prediction of a simple optically thick accretion disk model. The source is extended also at $1.3 \mathrm{~mm}$. The flux in a $28^{\prime \prime}$ beam is $540 \mathrm{mJy}$ (Hillenbrand et al. 1992), which corresponds to about $22 M_{\odot}$ in a region of about $0.34 \mathrm{pc}$ size. No compact $2.7 \mathrm{~mm}$ emission is detected by di Francesco et al. (1997).

The molecular survey of Hillenbrand (1995) yields a mass estimate of $M_{\mathrm{cl}}=1400 M_{\odot}$ in a region of $\sim 1.1 \mathrm{pc}$ size. Fuente et al. (1998) have observed the star at the IRAM-30 $\mathrm{m}$ telescope and detected emission both in the line and continuum, obtaining $M=11 M_{\odot}$ within 0.08 pc. On the other hand, Molinari et al. (1996) report no detection of $\mathrm{NH}_{3}(1,1)$ and $(2,2)$ line emission and of $\mathrm{H}_{2} \mathrm{O}$ maser emission.

(Fig. 9). In our NIR images, a conspicuous group of stars is detected close to the Herbig Be star and embed- ded in a diffuse nebulosity. The radius of the group is $r \sim 0.7 \mathrm{pc}$.

\subsection{AS 310 (PK 026+01.1; $P N V V$ 425)}

The $V$-magnitude and $(B-V)$ colour are from Shevchenko et al. (1993). The distance $(2500 \mathrm{pc})$ and the spectral type (B0) are from Henning et al. (1994).

The star is associated with the HII region S61 (Georgelin \& Georgelin 1970). Henning et al. (1994) observed the source at $1.3 \mathrm{~mm}$ and derived a gas mass of $30 M_{\odot}$. It is known to be a binary system (Bastian \& Mundt 1979) with separation 4.4 arcsec. Ageorges et al. (1997) high angular resolution NIR observations have revealed 4 more sources located close to the binary (all within $5^{\prime \prime}$ ) and conclude that there is a small cluster around the Herbig AeBe star.

AS 310 has a $1.3 \mathrm{~mm}$ flux of $190 \mathrm{mJy}$ (Henning et al. 1994), from which we infer a mass of $8 M_{\odot}$ in a region of $0.28 \mathrm{pc}$ size.

(Fig. 10). In our NIR image the field is extremely crowded, however a clear peak in the source surface 


$$
\mathrm{BD}+40^{\circ} 4124
$$
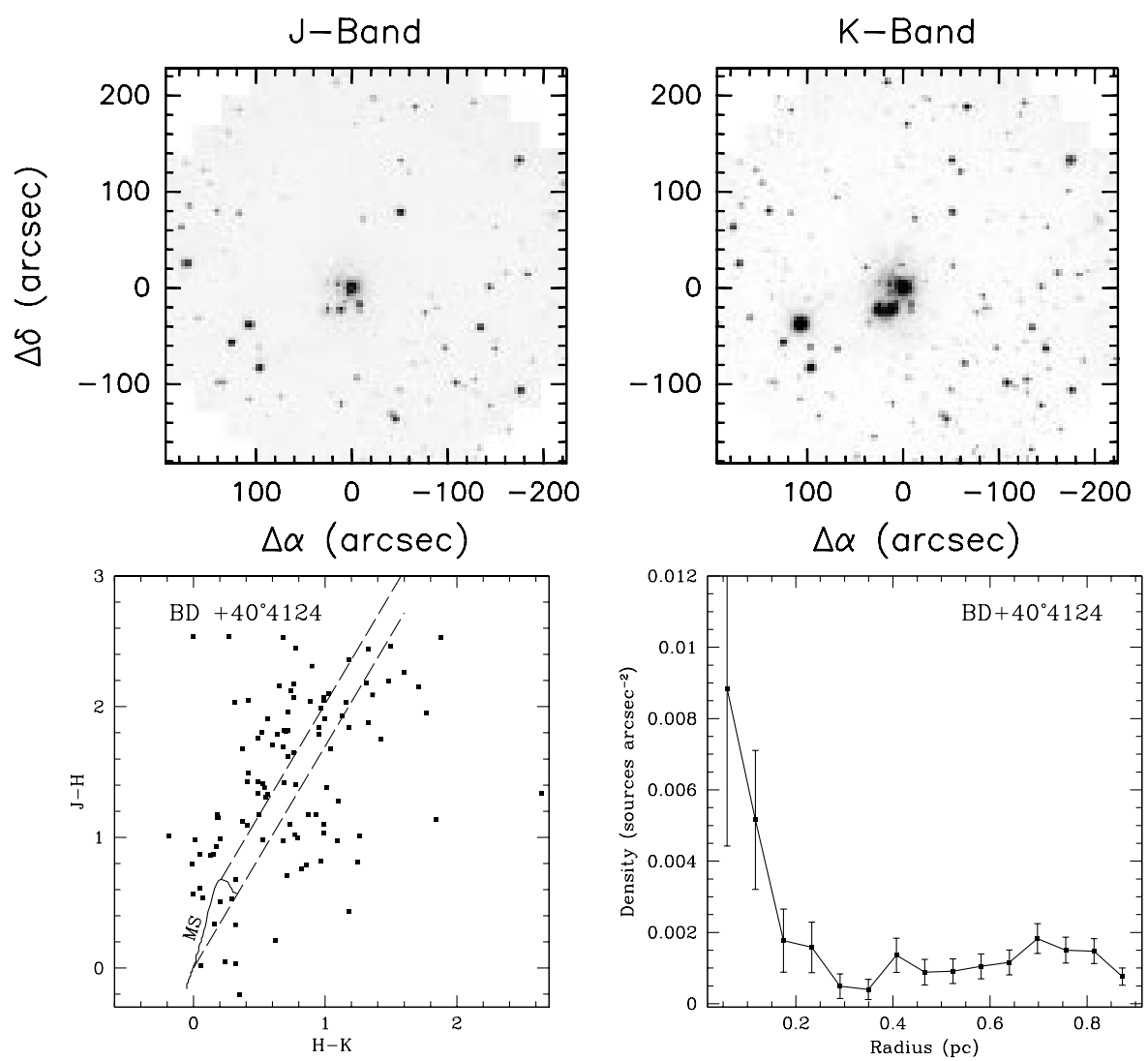

Fig. 15. BD $+40^{\circ}$ 4124. Top left: $J$-band image; top right: $K$-band image; bottom left: colour-colour diagram; bottom right: $K$-band source surface density profile

density profile is seen with a radius of $\sim 0.4$ pc. This finding suggests that Ageorges et al. (1997) are in fact detecting the brightest (and closer to the Herbig AeBe star) members of a conspicuous cluster.

\subsection{RNO 6 (HBC 334; IRAS 02130+5509)}

The star is from the Red and Nebulous Objects catalogue of Cohen (1980), who give the spectral type that we are using $(\mathrm{B} 1)$. The distance $(1600 \mathrm{pc})$ is from Scarrot et al. (1986). $V$ and $(B-V)$ from Herbig \& Bell (1988) catalogue. It is not included in the list of Finkenzeller \& Mundt (1984).

(Fig. 11). An arc-shaped nebula is detected at all NIR wavelegths. The source surface density profile shows a clear enhancement toward the central position. The radius of the density peak is $r \sim 0.3 \mathrm{pc}$.

\section{9. $H D$ 52721 (BD-1101747; $M W C$ 164; \\ IRAS 06594-1113; GU CMa; VDB 88)}

The $V$-magnitude and $(B-V)$ colour are from Shevchenko et al. (1993). The distance (1150 pc) is from Finkenzeller \&
Mundt (1984) and the spectral type (B2) from Hillenbrand et al. (1992).

New spectroscopic results (van den Ancker et al. 1997) confirm that the spectral type is B2Ve. Evidence from rotational velocity and the small IR excess supports the interpretation that it could be a classical Be star rather than a Herbig Be star. However, we detect a measurable excess of stars around it (Paper I) supporting the hypothesis that it is indeed a young star surrounded by a group of lower luminosity objects. The estimate of the distance $(1150 \mathrm{pc})$ comes from Herbst (1982) who assumed that the star is at the same distance of the nebulosity of CMa R1.

Fuente et al. (1998) have observed this object at mm wavelengths; they find that the star sits in a large cavity devoid of molecular gas.

(Fig. 12). Very crowded field with a marginally detected source enhancement with radius $r \sim 0.6 \mathrm{pc}$. 


\section{HD 37490}
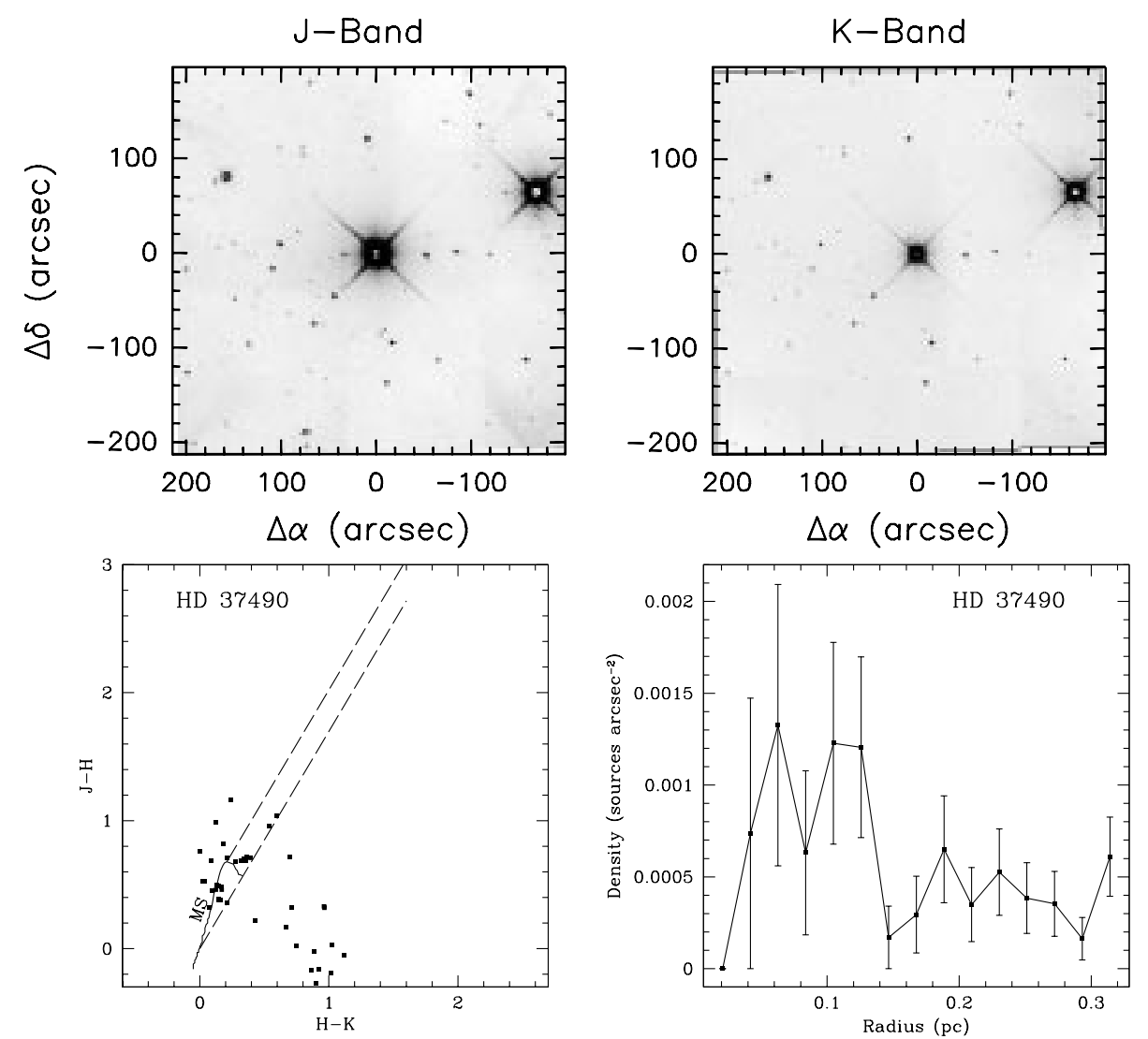

Fig. 16. HD 37490. Top left: $J$-band image; top right: $K$-band image; bottom left: colour-colour diagram; bottom right: $K$-band source surface density profile

\subsection{0. $B D+65^{\circ} 1637$ (NGC 7129; V361 Cep; HBC 730; $A S 475)$}

The $V$-magnitude, $(B-V)$ colour and spectral type (B2) are from Shevchenko et al. (1993). The distance $(1 \mathrm{kpc})$ is from Finkenzeller \& Mundt (1984).

This star is in the same molecular complex that harbors $\mathrm{LkH} \alpha 234$. Large scale observations of the molecular emission have shown that $\mathrm{BD}+65^{\circ} 1637$ (and most of the cluster, see below) are at the center of a region evacuated from the molecular material, while $\mathrm{LkH} \alpha 234$ is at the edge of a molecular ridge (Fuente et al. 1998). From line and continuum measurements the mass of gas and dust associated with the star is very modest $\left(M^{{ }^{13} \mathrm{CO}}=0.4 M_{\odot}\right.$, $\left.M_{\mathrm{CS}}=1.0 M_{\odot}, M_{\text {dust }}=0.6 M_{\odot}\right)$.

(Fig. 13). A clear enhancement of stars is detected between the two Herbig AeBe stars BD $+65^{\circ} 1637$ (the more massive visible star in the region) and $\operatorname{LkH} \alpha 234$, the brightest source in the field at $K$-band, located toward the north east with respect to the center. The peak of the stellar surface density profile centered on the B2 star has a radius of $\sim 0.4 \mathrm{pc}$. Diffuse emission is clearly detected around $\mathrm{LkH} \alpha 234$ and in a $\sim 2$ arcminute long filament, which is probably marking the interaction be- tween the radiation field of $\mathrm{BD}+65^{\circ} 1637$ and the surface of the molecular cloud that sorrounds the stellar group toward the south east. The large scale molecular observations of Fuente et al. (1998) confirmed that this structure is indeed tracing the edge of the molecular cloud.

\subsection{HD 216629 (BD+61 2361; $M W C$ 1075; IL Cep)}

The $V$-magnitude and $(B-V)$ colour are from Shevchenko et al. (1993). The distance $(725 \mathrm{pc})$ is from Garmany (1973) and the spectral type that we assume (B2) is from Finkenzeller \& Mundt (1984).

The NIR images of Pirzkal et al. (1997) reveal a companion at $6.96^{\prime \prime}$ with no difference at $K$ between the primary and the companion star.

(Fig. 14). Very crowded field with a moderate source surface density enhancement with $r \sim 0.1$ pc detected. The bright companion detected by Pirzkal et al. (1997) is clearly revealed. 

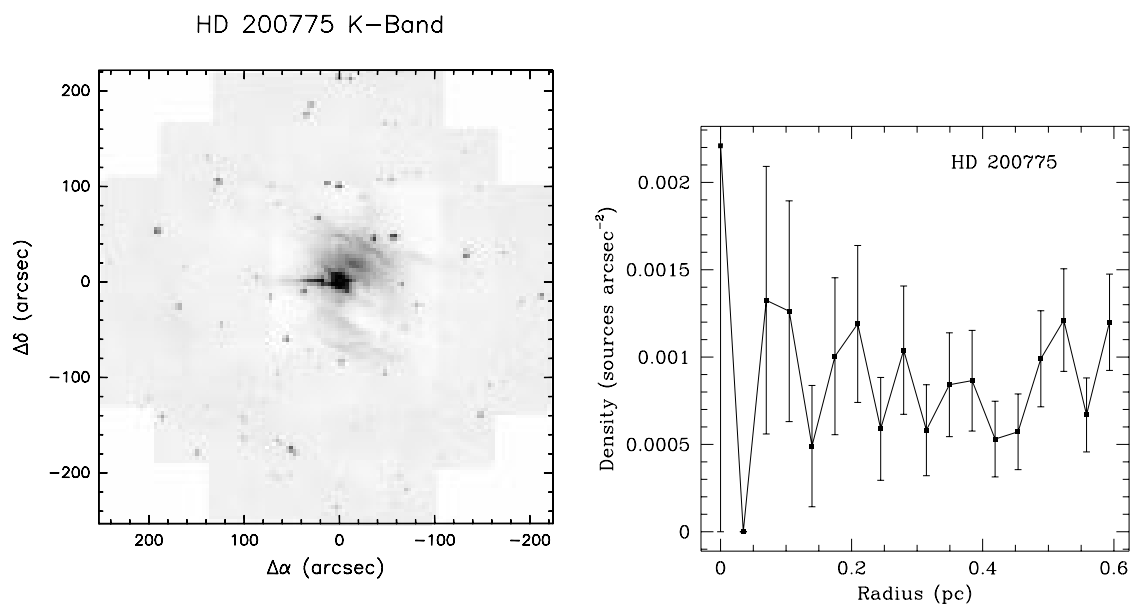

Fig. 17. HD 200775. Left: $K$-band image; right: $K$-band source surface density profile
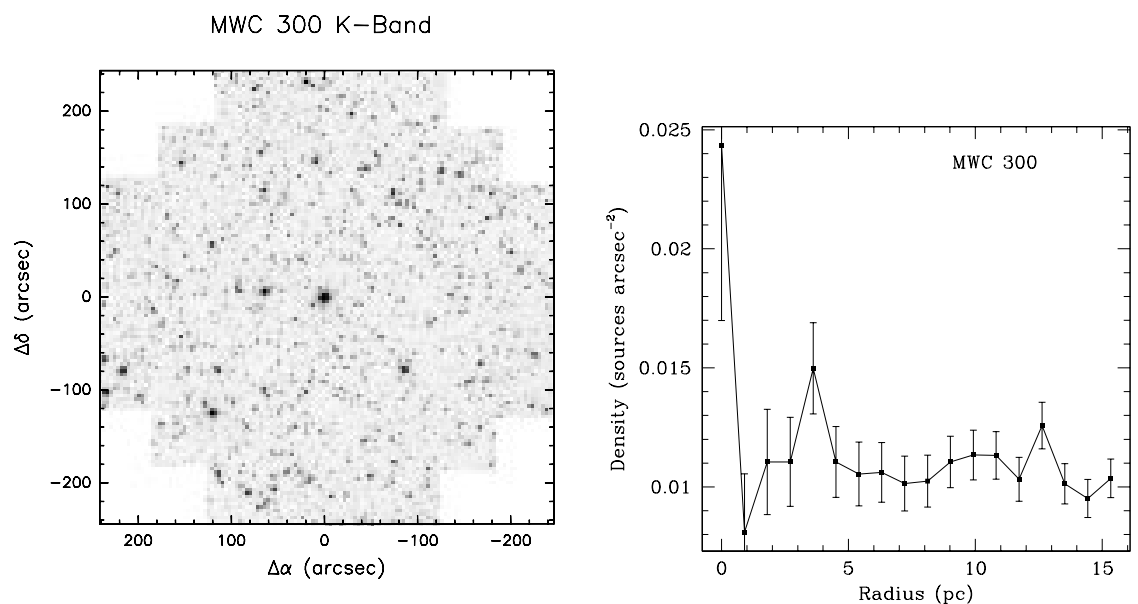

Fig. 18. MWC 300. Left: $K$-band image; right: $K$-band source surface density profile
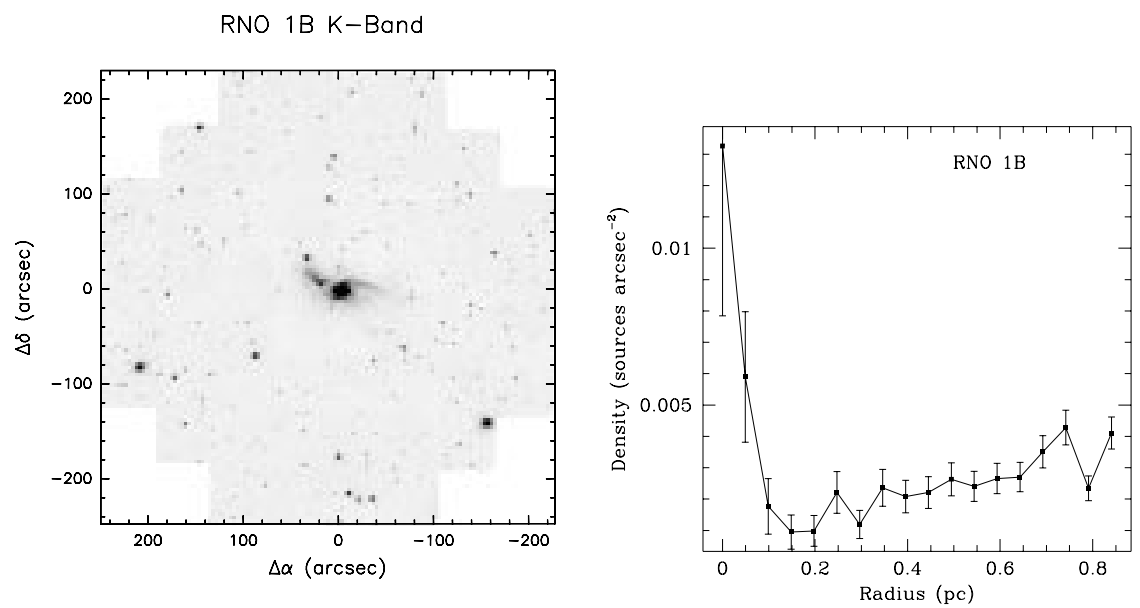

Fig. 19. RNO 1B. Left: $K$-band image; right: $K$-band source surface density profile 


\section{HD 259431}
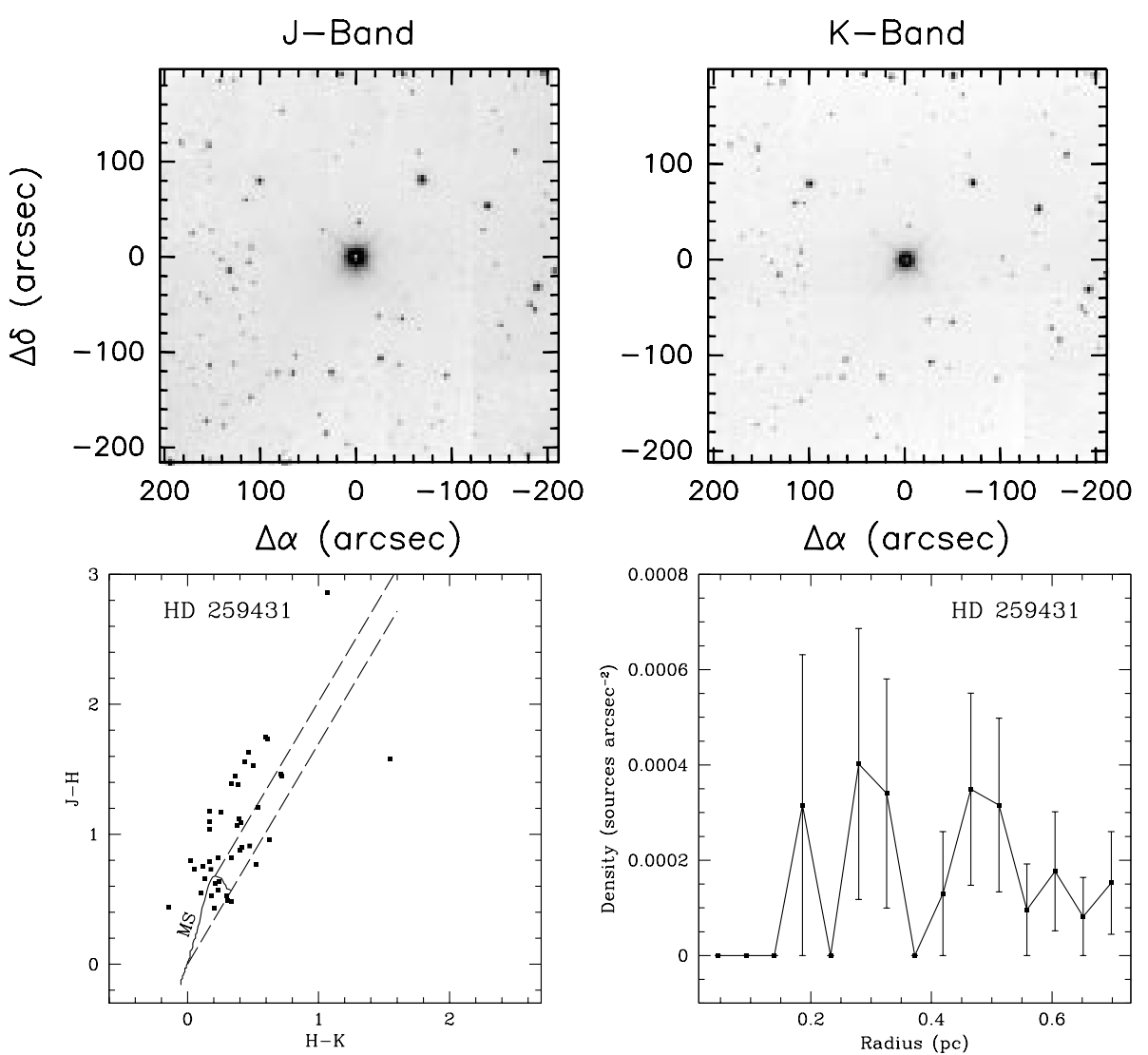

Fig. 20. HD 259431. Top left: $J$-band image; top right: $K$-band image; bottom left: colour-colour diagram; bottom right: $K$-band source surface density profile

\subsection{2. $B D+40^{\circ} 4124$ (MWC 340; V 1685 Cyg; He 3-1882)}

The $V$-magnitude and $(B-V)$ colour are from Shevchenko et al. (1993). The distance $(1000 \mathrm{pc})$ and spectral type (B2) from Finkenzeller \& Mundt (1984).

The object is detected at $1.3 \mathrm{~mm}$ (Hillenbrand et al. 1992); the corresponding circumstellar mass is about $1.5 M_{\odot}$ in a region of $0.14 \mathrm{pc}$ size. In the $2.7 \mathrm{~mm}$ observations of di Francesco et al. (1997) the star itself is not detected. However, they detect a strong, unresolved source $0.9^{\prime \prime}$ north of V1318 Cyg S. This source is also closest to the emission peak at $800 \mu \mathrm{m}$ (Aspin et al. 1994), and the center of the outflow and $\mathrm{H}_{2} \mathrm{O}$ maser emission (Palla et al. 1995), and it is believed to be one of the youngest sources in the region.

The molecular survey of Hillenbrand (1995) yields a mass estimate of $M_{\mathrm{cl}}=862 M_{\odot}$ in a region of $=0.7 \mathrm{pc}$ size.

(Fig. 15). An embedded rich group is detected within $r \sim 0.2$ pc from the Herbig Be star, embedded in a diffuse nebulosity. There are at least three bright members in the group at $K$-band. This region has been studied in detail by Aspin et al. (1994), Hillenbrand et al. (1995) and Palla et al. (1995). The group is embedded in a compact molec- ular clump which prevents the detection of at least $70 \%$ of the sources at optical $(R)$ wavelengths.

\subsection{HD 37490 ( $\omega$ Ori; $M W C 117 ; B D+04^{\circ} 1002$;} HR 1934; IRAS 05365+0405; SAO 113001; HIP 26594)

The $V$-magnitude and $(B-V)$ colour are from Berrilli et al. (1992), the distance (360 pc) from Racine (1968), spectral type (B3) from Finkenzeller \& Mundt (1984).

Hillenbrand et al. (1992) give a distance of $360 \mathrm{pc}$ and a spectral type B2; from the shape of the SED they classify it as a Group III Herbig AeBe star. Hipparcos measurements (van den Anker et al. 1998) give a parallax of $2 \pm$ 0.9 mas and a distance $>210 \mathrm{pc}$.

(Fig. 16). The bright Herbig AeBe star is surrounded by a faint nebulosity. A stellar density enhancement with radius $r \sim 0.14 \mathrm{pc}$ is detected.

\subsection{HD 200775 (BD+67 1283; MWC 361; HBC 726)}

The $V$-magnitude and $(B-V)$ colour are from Shevchenko et al. (1993). The distance $(600 \mathrm{pc})$ and the spectral type (B3) are from Finkenzeller \& Mundt (1984). 


\section{XY Per}
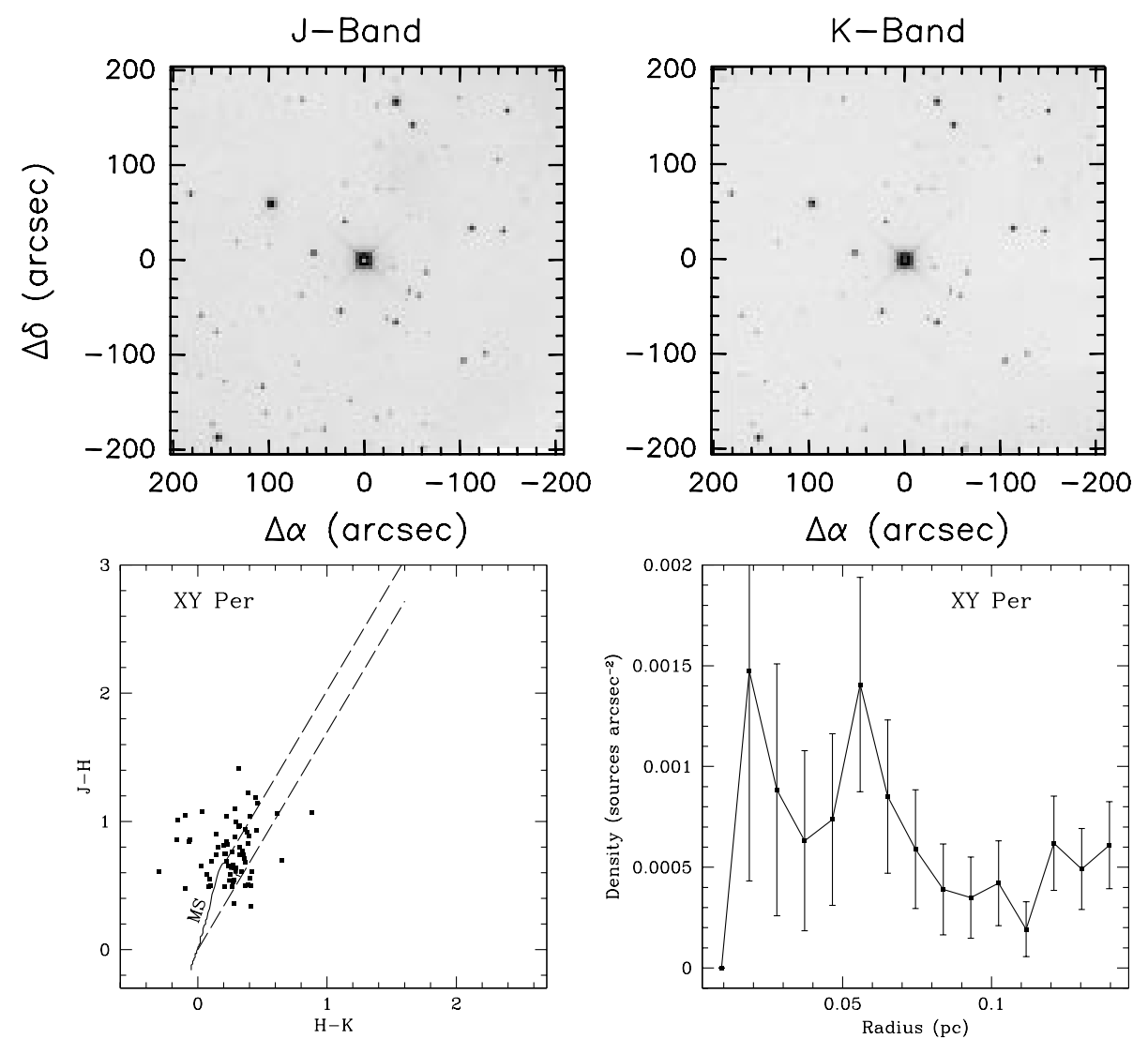

Fig. 21. XY Per. Top left: $J$-band image; top right: $K$-band image; bottom left: colour-colour diagram; bottom right: $K$-band source surface density profile

The molecular survey of Hillenbrand (1995) yields a mass estimate of $M_{\mathrm{cl}}=720 M_{\odot}$ in a region of $\sim 0.8 \mathrm{pc}$ size. High resolution observations by Fuente et al. (1998) show that on a scale of $0.08 \mathrm{pc}$ the amount of gas and dust is reduced to $\sim 1.5 M_{\odot}$.

(Fig. 17). The star is surrounded by a bright nebulosity, which is probably marking the region where the stellar radiation field interacts with the surrounding molecular material. No source surface density enhancement is detected.

\subsection{MWC 300 (V431 Sct; IRAS 18267-0606; HBC 283; HIP 90617)}

Both the distance and the spectral classification are very uncertain. We take a distance of $15.5 \mathrm{kpc}$ and a spectral type Be, as reported by Leinert et al. (1997).

(Fig. 18). Our image shows a very crowded field, a stellar density peak with radius $r \sim 4$ pc (assuming a distance of $15.5 \mathrm{kpc}$ ) is possibly detected.

\subsection{6. $R N O 1 B$}

Yang et al. (1991) classify this star as a FU Orionis star, the distance is estimated to be $850 \mathrm{pc}$. The spectral classification is very uncertain (Be-type). The infrared source is coincident with an ammonia clump (Estarella et al. 1993) with a size of $0.5 \times 0.2 \mathrm{pc}$ and an estimated $\mathrm{H}_{2}$ column density of $10^{23} \mathrm{~cm}^{-2}$. Anglada et al. (1994) report the detection of a group of compact radio sources, none of which is coincident with RNO $1 \mathrm{~B}$ or RNO $1 \mathrm{C}$, which are the brightest objects at $2.2 \mu \mathrm{m}$.

(Fig. 19). The star is surrounded by a bright extended nebulosity. Within the nebula a small group of stars with $r \sim 0.15 \mathrm{pc}$ is detected. The field stars surface density appears to increase away from the Herbig AeBe star, suggesting that localized extinction is present. The size of the cluster appears to be consistent with that of the ammonia clump detected by Estarella et al. 1993). The presence of localized dense molecular material could explain the lack of background stars close to the central object. However, the expected extinction provided by the molecular gas would prevent the detection of young stars embebbed inside the clump, thus the most plausible 


\section{$L k H \alpha 25$}
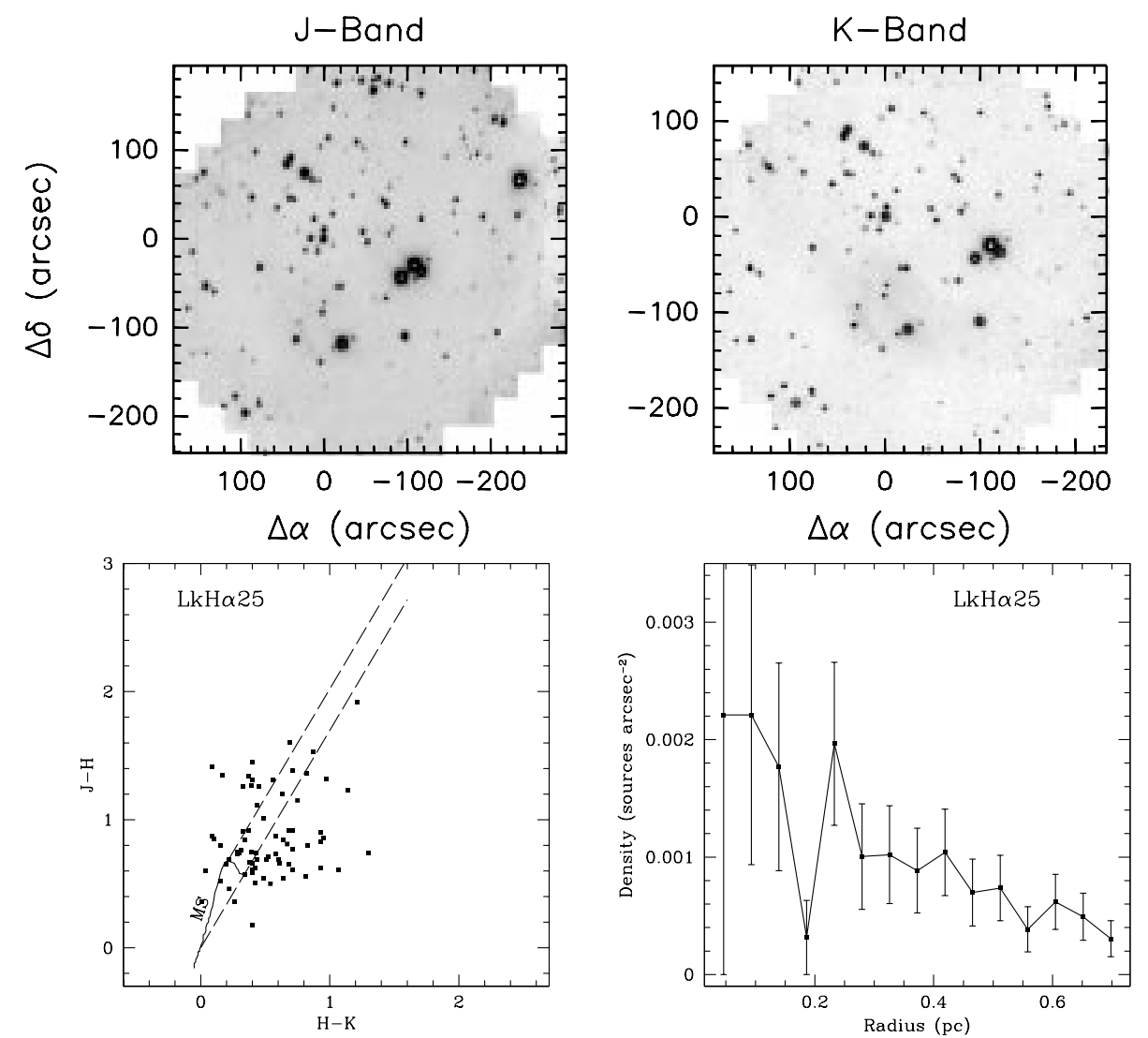

Fig. 22. LkH $\alpha$ 25. Top left: $J$-band image; top right: $K$-band image; bottom left: colour-colour diagram; bottom right: $K$-band source surface density profile

explanation is that a cluster of young stars is emerging from the observer's side of the cloud.

\subsection{HD 259431 (BD+10॰1172; $M W C 147 ; N G C 2247)$}

The $V$-magnitude and $(B-V)$ colour are from Shevchenko et al. (1993). The distance $(800 \mathrm{pc})$ is from Finkenzeller \& Mundt (1984) and the spectral type (B5) from Hillenbrand (1995).

The star is included in the Hipparcos data (van den Ancker 1988), however, only a lower limit to the distance $(D>130 \mathrm{pc})$ is given. It is classified as a B1Ve star (with $\log T_{\text {eff }}=4.41 \mathrm{~K}$ ) in Monoceros (associated with L 1605, NGC 2247 and Mon R1).

The molecular survey of Hillenbrand (1995) yields a mass estimate of $M_{\mathrm{cl}}=480 M_{\odot}$ in a region of $=0.8 \mathrm{pc}$ size.

(Fig. 20). In our images the star appears to be isolated and surrounded by a faint infrared nebulosity.

\subsection{XY Per (HD 275877; $A D S$ 2788; $\left.B D+38^{\circ} 811\right)$}

The $V$-magnitude and $(B-V)$ colour are from Shevchenko et al. (1993). The distance $(160 \mathrm{pc})$ is from Cohen (1973), and the spectral type (B6) from Finkenzeller \& Mundt (1984). The distance is uncertain. Hipparcos data (van den Ancker et al. 1998) give a distance of $120_{-36}^{+88} \mathrm{pc}, \mathrm{A} 2 \mathrm{II}+\mathrm{B} 6 \mathrm{e}, \log \left(T_{\text {eff }}\right)=4.15$. NIR shiftand-add imaging finds a companion at $1.2^{\prime \prime}(192 \mathrm{AU}$ at the assumed distance) with no $K$ magnitude difference between primary and companion (Pirzkal et al. 1997).

(Fig. 21). Although the field does not appear to be crowded, the source surface density profile reveals only a moderate enhancement of sources within $r \sim 0.08 \mathrm{pc}$ from the Herbig AeBe star.

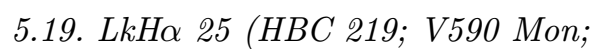 IRAS 06379+0950)}

The $V$-magnitude and $(B-V)$ colour are from Shevchenko et al. (1993). The distance $(800 \mathrm{pc})$ is from Finkenzeller \& Mundt (1984) and the spectral type (B7) from Hillenbrand (1995). Hillenbrand et al. (1992) classify this 


\section{HD 250550}
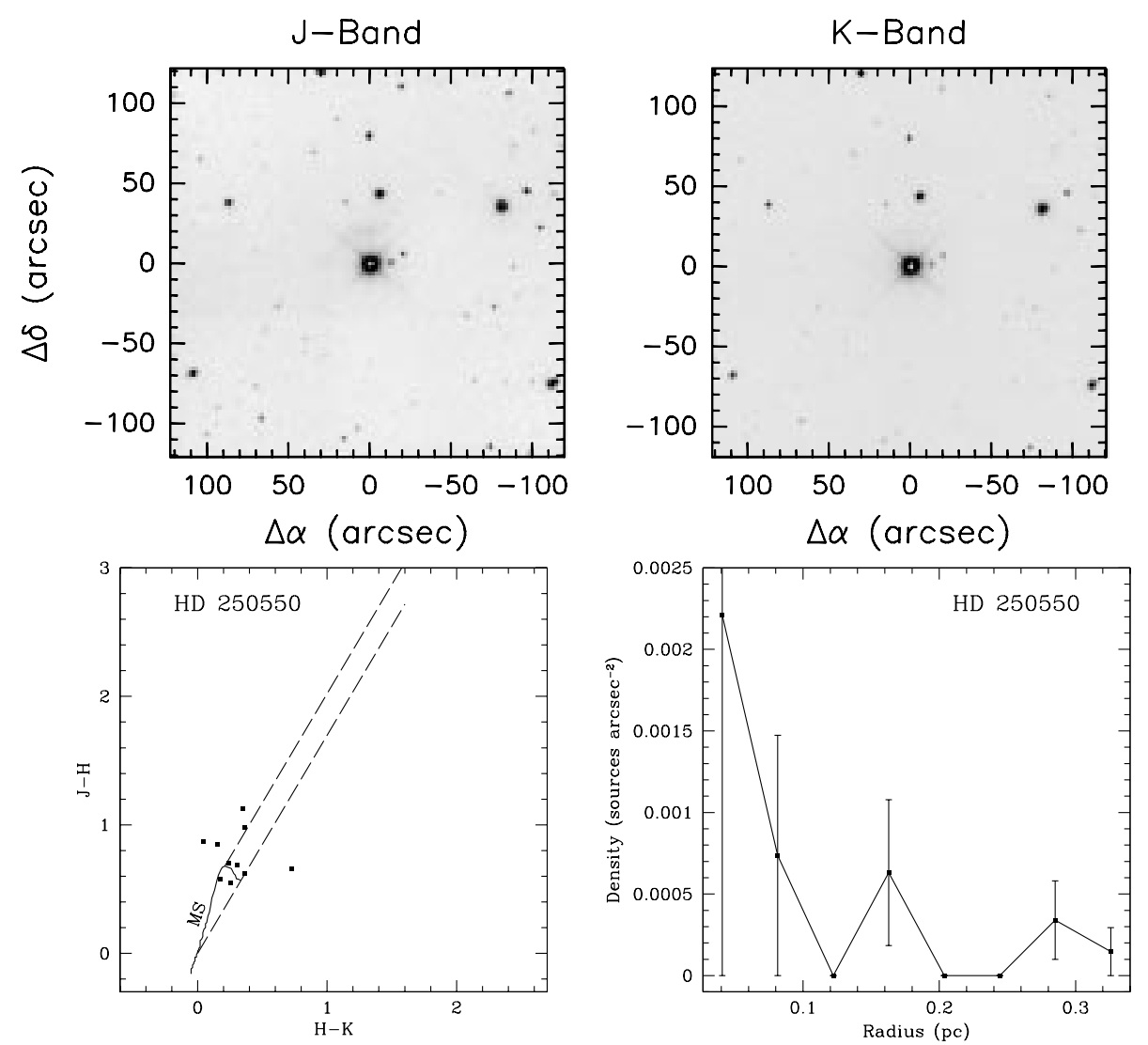

Fig. 23. HD 250550. Top left: $J$-band image; top right: $K$-band image; bottom left: colour-colour diagram; bottom right: $K$-band source surface density profile

star as a Group II star, and spectral type A0, while Finkenzeller \& Mundt (1984) classify it as a B8 star.

(Fig. 22). Crowded field with several bright stars and faint diffuse nebulosity. A small group of stars is detected within $r \sim 0.3 \mathrm{pc}$ from the Herbig AeBe star.

\subsection{0. $H D 250550 \quad\left(B D+16^{\circ} 974 ; M W C 789\right)$}

The $V$-magnitude and $(B-V)$ colour are from Shevchenko et al. (1993). The distance (700 pc) is from Finkenzeller $\&$ Mundt (1984), the spectral type (B7) from Hillenbrand et al. (1992).

The star is included in the Hipparcos data of van den Ancker et al. (1998), but only a lower limit to the distance $(>110 \mathrm{pc})$ is given.

The star is not detected at $1.3 \mathrm{~mm}$ (Hillenbrand et al. 1992); the corresponding upper limit on the circumstellar mass is $0.13 M_{\odot}$ in a region of $0.1 \mathrm{pc}$ size. The molecular survey of Hillenbrand (1995) yields a mass estimate of $M_{\mathrm{cl}}=140 M_{\odot}$ in a region of $0.5 \mathrm{pc}$ size. Higher resolution observations by Fuente et al. (1998) show that on a scale of $0.08 \mathrm{pc}$ the amount of gas and dust is reduced to $\sim 5 M_{\odot}$.
(Fig. 23). Close to the bright Herbig AeBe star there is a faint nebulosity to the north. No source density enhancement is detected.

\subsection{1. $L k H \alpha 215$ (NGC 2245)}

The $V$-magnitude and $(B-V)$ colour are from Shevchenko et al. (1993). The distance (800 pc) and spectral type (B7) from Finkenzeller \& Mundt (1984).

Detected at submm wavelengths by Mannings (1994), who concluded from modelling of the SED that a second dust component in addition to an optically thick disk is required to explain the excess of emission at $450 \mu \mathrm{m}$.

The molecular survey of Hillenbrand (1995) yields a mass estimate of $M_{\mathrm{cl}}=312 M_{\odot}$ in a region of $0.5 \mathrm{pc}$ size. Higher resolution observations by Fuente et al. (1998) show that on a scale of $0.08 \mathrm{pc}$ the amount of gas and dust is reduced to $\sim 4 M_{\odot}$.

(Fig. 24). A small group of stars is detected within $r \sim 0.18 \mathrm{pc}$ from the Herbig AeBe star. The group is embedded in an arc shaped nebulosity. 


\section{$L k H \alpha 215$}
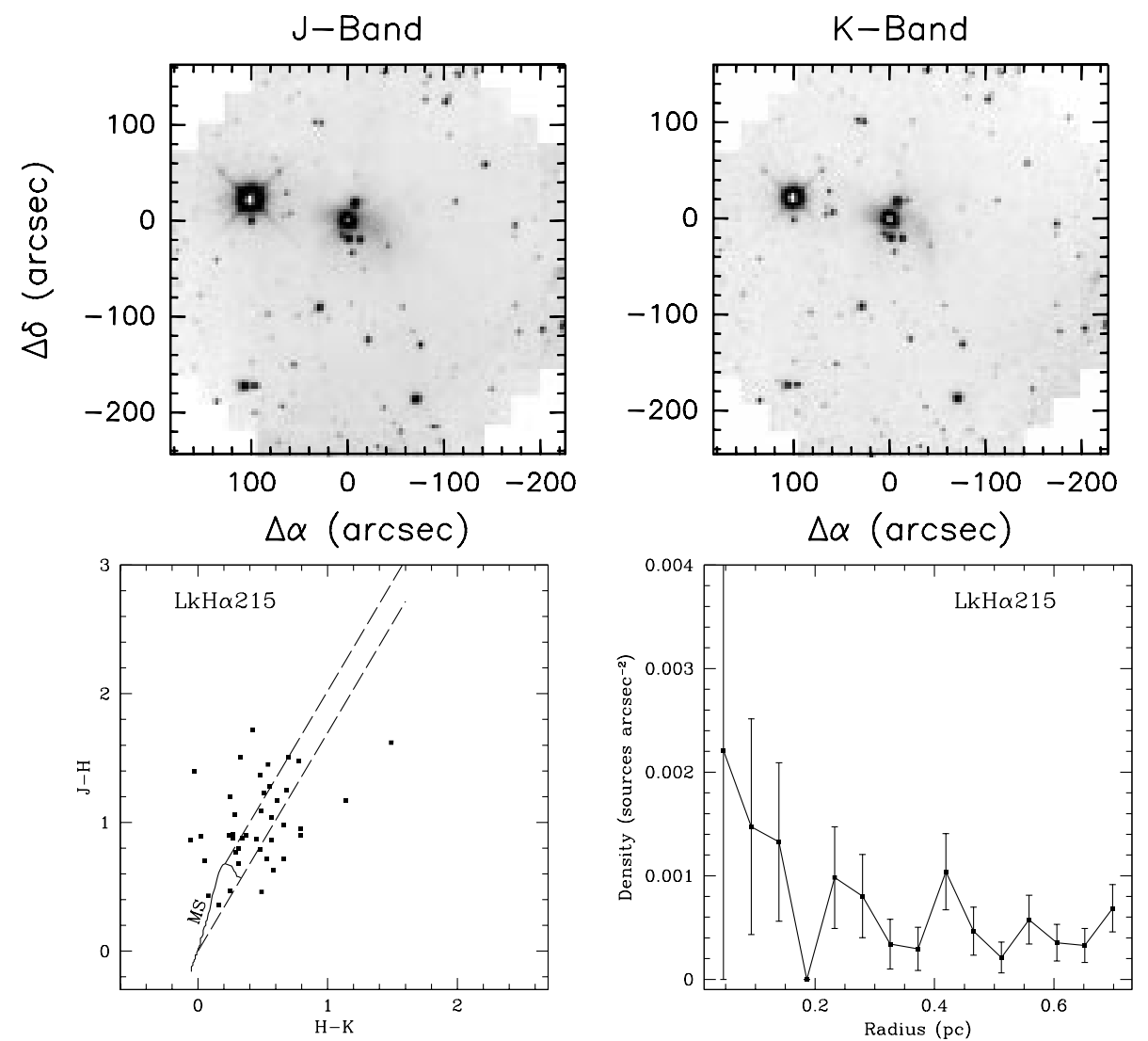

Fig. 24. LkH $\alpha$ 215. Top left: $J$-band image; top right: $K$-band image; bottom left: colour-colour diagram; bottom right: $K$-band source surface density profile

\subsection{2. $L k H \alpha 257$ (HBC 312; IRAS 21523+4657)}

The $V$-magnitude and $(B-V)$ colour are from Shevchenko et al. (1993). The distance $(800 \mathrm{pc})$ and the spectral type (B8) are from Damiani et al. (1994).

$\mathrm{Li}$ et al. (1994) observed this source but reported no detection of companions or extended infrared emission.

(Fig. 25). In our large field infrared image, the stars in the field appear unevenly distributed, but no clear central peak is revealed in the source surface density profile.

\subsection{3. $B D+61^{\circ} 154$ (V594 Cas; IRAS 00403+6138; MWC 419; HIP 3401; HBC 330)}

The $V$-magnitude and $(B-V)$ colour are from Shevchenko et al. (1993). Distance (650 pc) and spectral type (B8) are from Finkenzeller \& Mundt (1984). The Hipparcos parallax of $3.3 \pm 1.6$ mas (van den Anker 1998) yields a lower limit on the distance of $D>120 \mathrm{pc}$ (which is consistent with the distance that we assume).

Hillenbrand et al. (1992) classify this as a Group I star. Li et al. (1994) observed the source to search for faint companions but their NIR images did not revealed any close companion or extended emission.

Millimeter observations by Hillenbrand et al. (1992) give a mass of about $0.16 M_{\odot}$ in a region of about $0.1 \mathrm{pc}$ size. The molecular survey of Hillenbrand (1995) yields an upper limit to the total mass of $M_{\mathrm{cl}}<180 M_{\odot}$ in a region of 0.2 pc size. Higher resolution observations by Fuente et al. (1998) show that on a scale of $0.08 \mathrm{pc}$ the amount of gas and dust is reduced to $\sim 1 M_{\odot}$.

(Fig. 26). The source surface density is uniform across the field (within the uncertainties), no density enhancement is detected around the Herbig AeBe star.

\subsection{VY Mon (HBC 202; IC 446)}

The $V$-magnitude and $(B-V)$ colour are from Shevchenko et al. (1993). The distance and spectral type that we adopt are from Damiani et al. (1994). The distance is that of the Monoceros OB1 association of which the star is assumed to be a member. The spectral type (B8) is from Thé et al. (1994).

(Fig. 27). A small group of stars within $r \sim 0.25 \mathrm{pc}$ is detected around the Herbig AeBe star. Some of the stars 

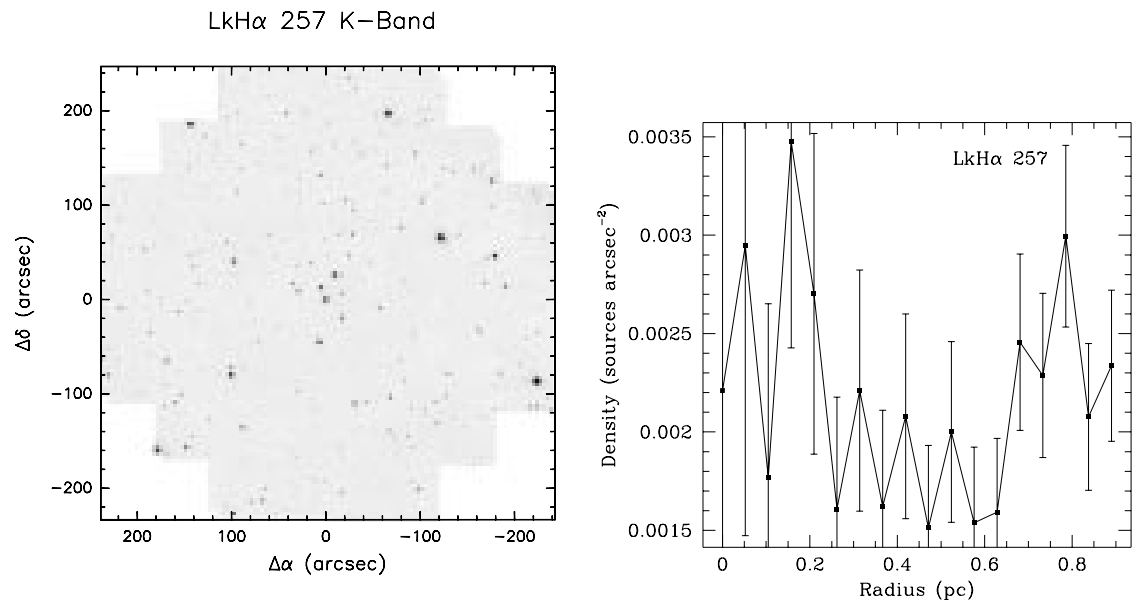

Fig. 25. LkH $\alpha$ 257. Left: $K$-band image; right: $K$-band source surface density profile
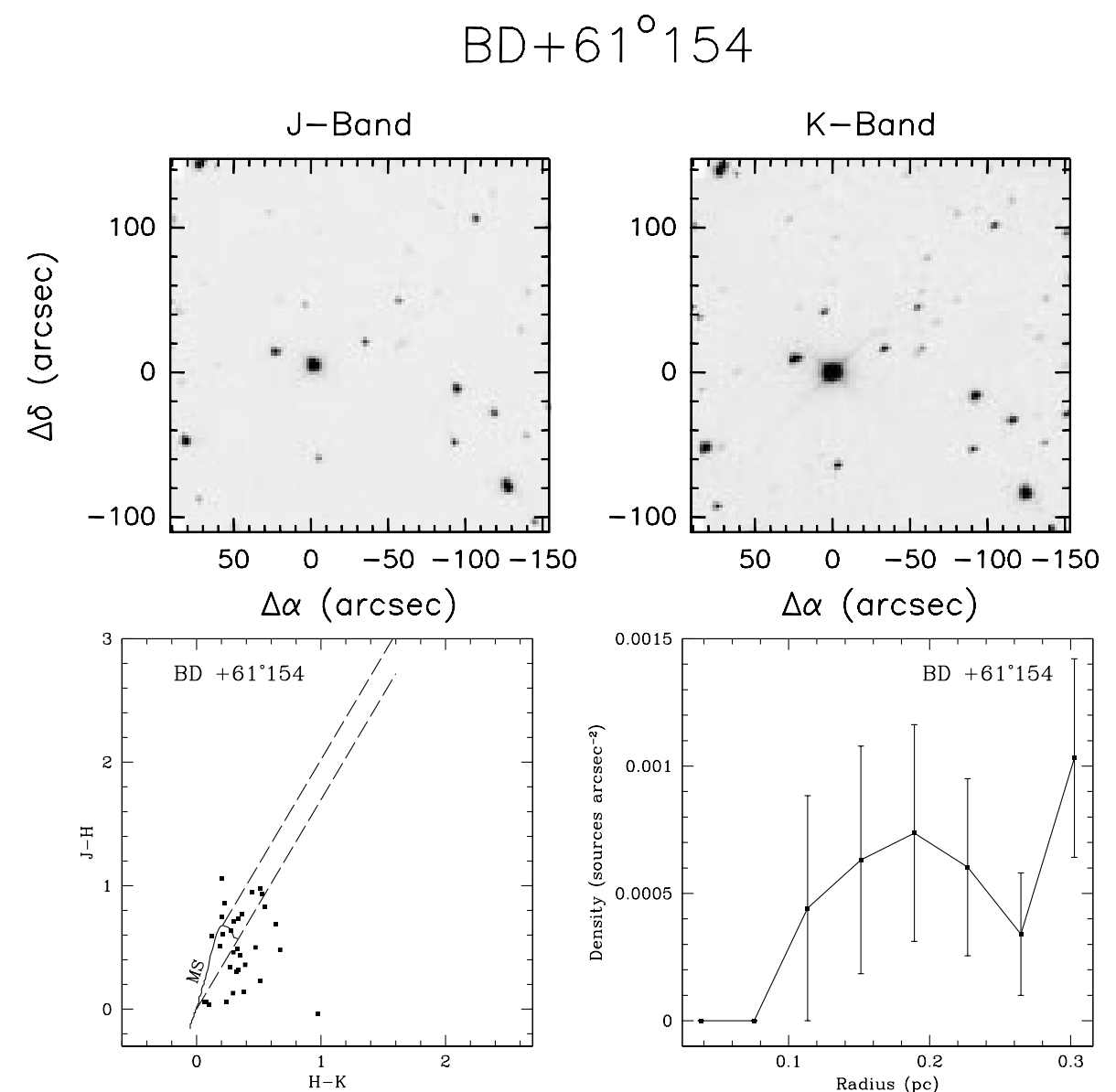

Fig. 26. $\mathbf{B D}+61^{\circ}$ 154. Top left: $J$-band image; top right: $K$-band image; bottom left: colour-colour diagram; bottom right: $K$-band source surface density profile 


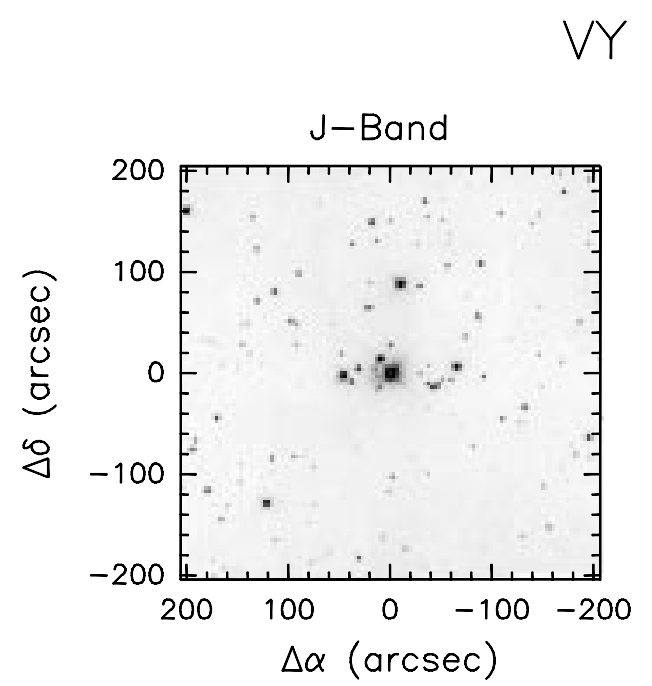

Mon
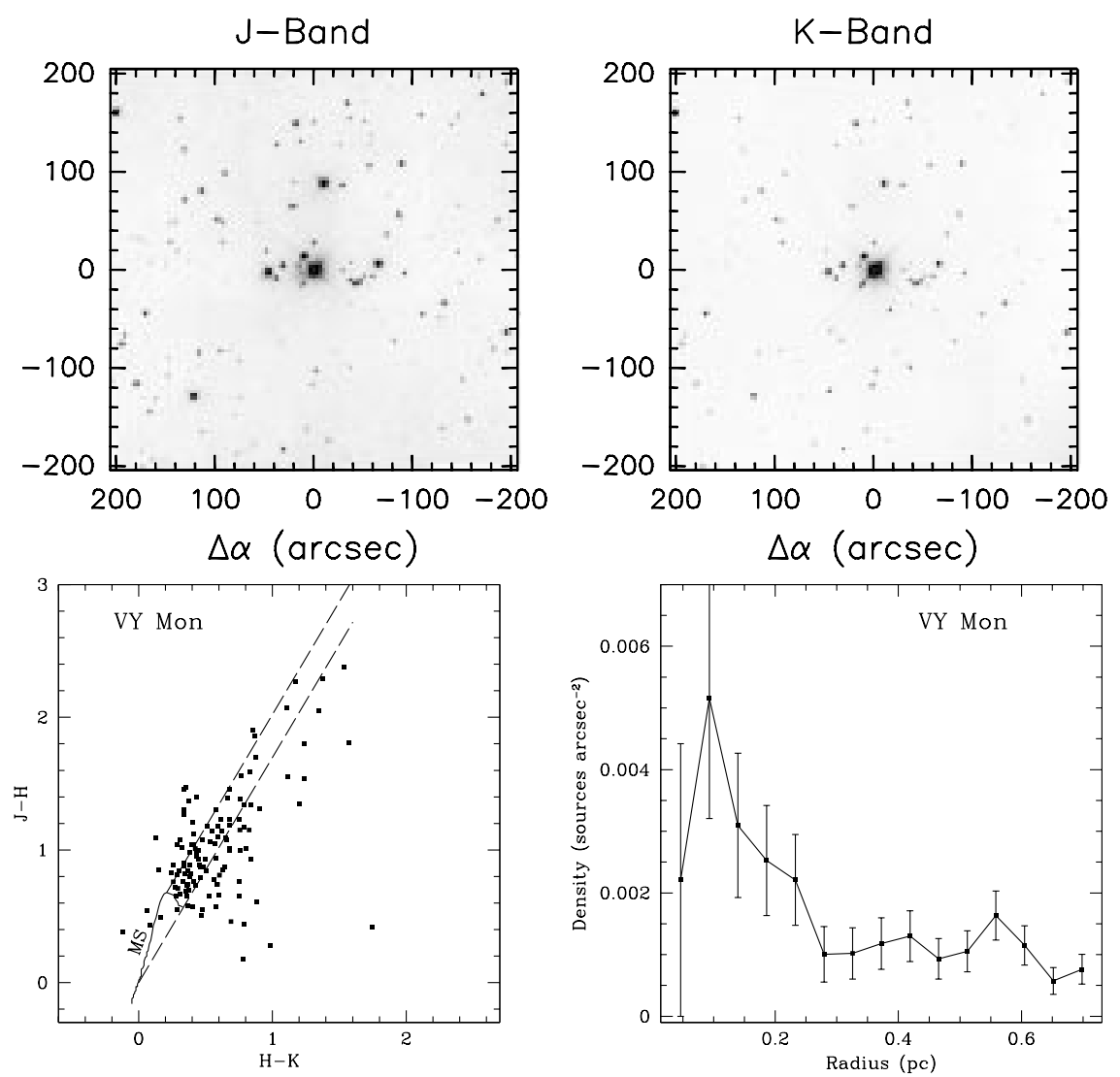

Fig. 27. VY Mon. Top left: $J$-band image; top right: $K$-band image; bottom left: colour-colour diagram; bottom right: $K$-band source surface density profile
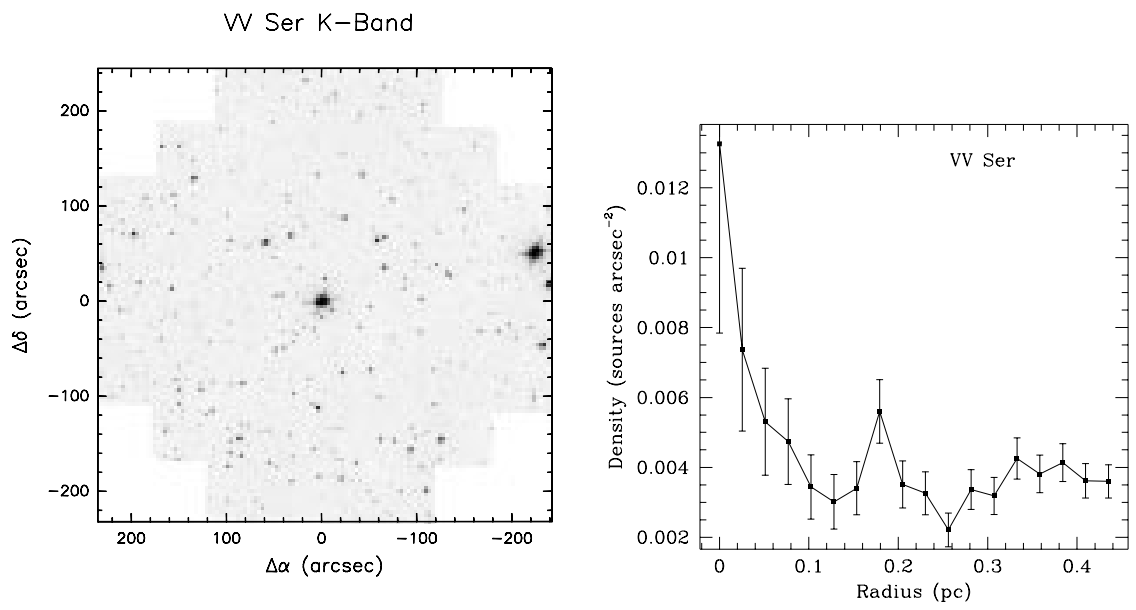

Fig. 28. VV Ser. Left: $K$-band image; right: $K$-band source surface density profile 


\section{380 Ori}
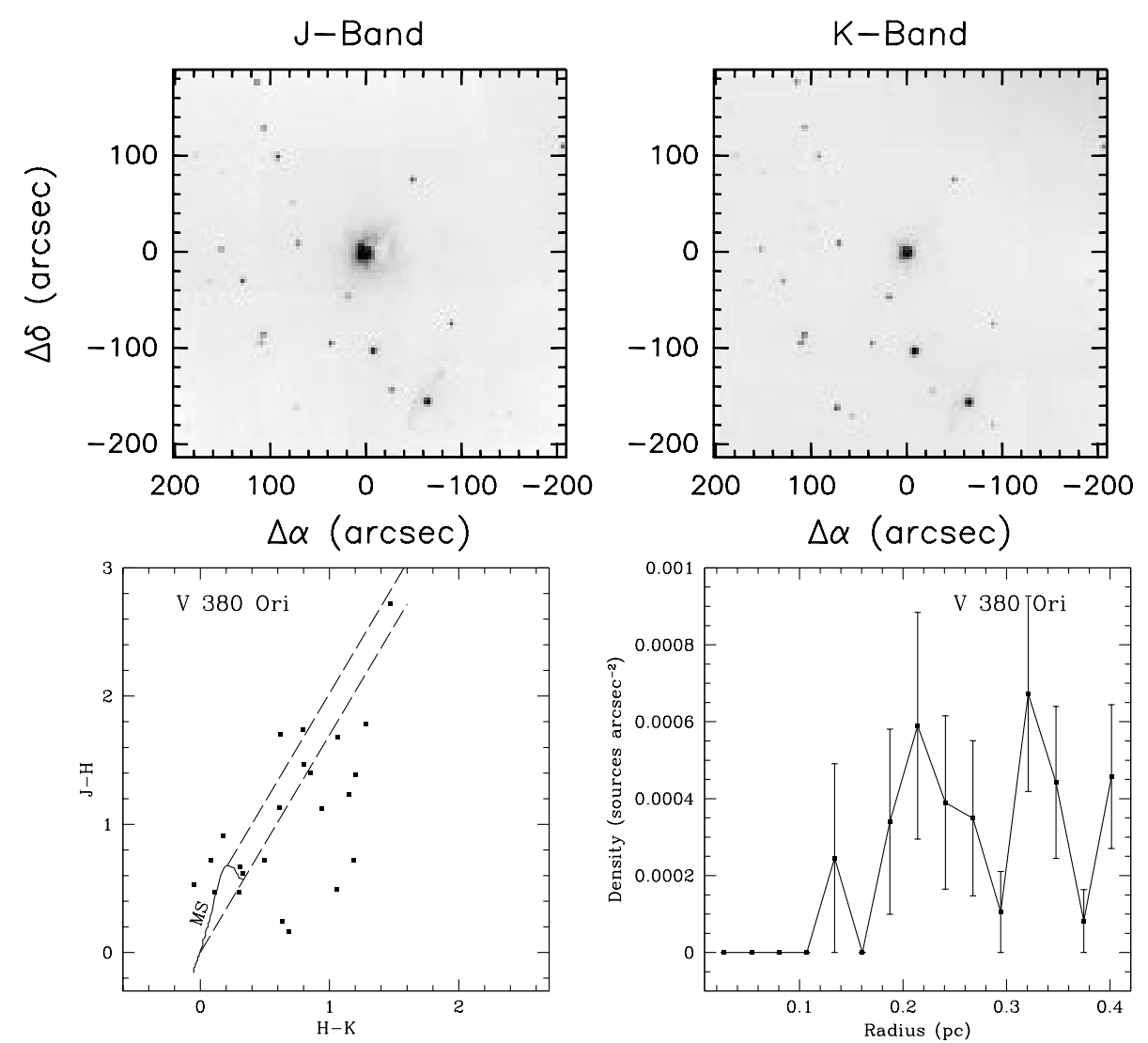

Fig. 29. V 380 Ori. Top left: $J$-band image; top right: $K$-band image; bottom left: colour-colour diagram; bottom right: $K$-band source surface density profile

in the field show infrared excess and a consistent amount of extinction.

\subsection{5. $V V$ Ser (HBC 282; IRAS 18262+0006)}

The $V$-magnitude and $(B-V)$ colour are from Shevchenko et al. (1993). The distance (440 pc) and the spectral type (B9) that we assume are from Hillenbrand et al. (1992). However, spectral type (and distance) are rather uncertain. Finkenzeller \& Mundt (1984) give B1/B3; Hamann \& Persson (1992) B5; Chavarria-K et al. (1988) A2 and $240 \mathrm{pc}$. All these different classifications are based on spectra, but use different lines.

Li et al. (1994) observed the source in their NIR survey for faint companions. In the $H$-band contour plot that they show there are 6 stars within $\sim 15^{\prime \prime}$ from the Herbig AeBe star. However, in spite of the fact that the closest companion is at $\sim 3350 \mathrm{AU}$ from the star, it is not detected in the search for close companions of Leinert et al. (1997), which should include all companions closer than $3600 \mathrm{AU}$.
The star is not detected in the millimeter (Hillenbrand et al. 1992). An estimate of the mass within $0.03 \mathrm{pc}$ gives $<0.025 M_{\odot}$ (Natta et al. 1997).

(Fig. 28). Our $K$-band image is completely consistent with the images of Li et al. (1994), and we detect all their six sources. A clear source surface density enhancement is detected around the star with $r \sim 0.1 \mathrm{pc}$.

\subsection{V380 Ori $\left(B D-06^{\circ} 1253 ; M W C 765\right.$; Haro 4-235)}

The $V$-magnitude and $(B-V)$ colour are from Shevchenko et al. (1993). The distance (460 pc) and spectral type (B9) from Hillenbrand et al. (1992).

The Hipparcos parallax (van den Ancker et al. 1998) is not determined with sufficient accuracy to derive a reliable estimate of the distance of the object.

In their survey, Leinert et al. (1997) find a companion at $0.134^{\prime \prime}\left(71 \mathrm{AU}\right.$ ) with a luminosity of $\sim 50 L_{\odot}$ (corresponding to another young intermediate mass star in the region).

There is a weak $1.3 \mathrm{~mm}$ emission associated to this star (Henning et al. 1994); the corresponding amount of 


\section{$\checkmark 1012$ Ori}
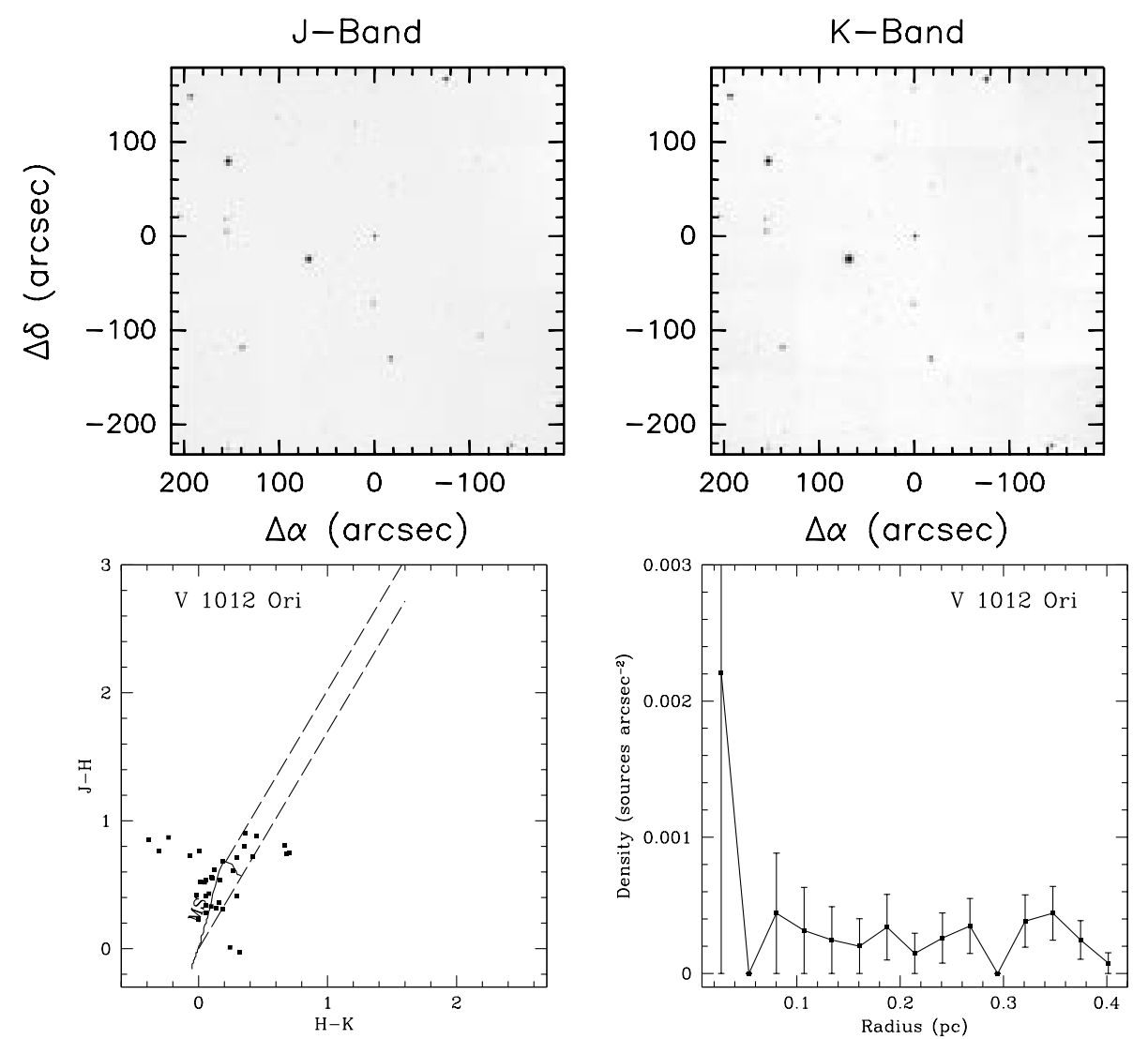

Fig. 30. V 1012 Ori. Top left: $J$-band image; top right: $K$-band image; bottom left: colour-colour diagram; bottom right: $K$-band source surface density profile

circumstellar gas and dust is rather small $\left(0.01 M_{\odot}\right.$ in a region of $0.05 \mathrm{pc}$ size).

(Fig. 29). The Herbig AeBe star is surrounded by a diffuse nebulosity, which might prevent the detection of faint companions close to the star. The source surface density profile does not show any enhancement close to the central position. Many NIR excess and reddened stars are detected in the field.

\subsection{1012 Ori (HBC 431; IRAS 05090-0226)}

To our knowledge, there is no measurements of $V$ and $(B-V)$ in the literature, hence it has not been possible to derive the stellar parameters as described above. The distance (460 pc) and spectral type (B9) are from Herbig \& Bell (1988) catalogue.

(Fig. 30). Very few stars in the field, no group detected.

\subsection{8. $L k H \alpha 218$}

The $V$-magnitude and $(B-V)$ colour are from Shevchenko et al. (1993). The distance $(1150 \mathrm{pc})$ is from Herbst et al. (1982) and spectral type (B9) from Hillenbrand et al. (1992).

(Fig. 31). Crowded field, no stellar density enhancement detected.

\subsection{9. $A B$ Aur (HD 31293; $B D+30^{\circ} 741 ; M W C$ 93)}

The $V$-magnitude and $(B-V)$ colour are from Shevchenko et al. (1993). The distance $(160 \mathrm{pc})$ from Finkenzeller \& Mundt (1984), the spectral type (A0) from Cohen \& Kuhi (1979).

The distance and physical parameters that we adopt are in agreement with the Hipparcos estimates: $d=$ $144 \mathrm{pc}$, Spectral type A0Ve+sh, $\log \left(T_{\text {eff }}\right)=4.00$, $\log (L)=1.72 L_{\odot}($ van den Ancker 1997).

$\mathrm{AB}$ Aur is not resolved at 50 and $100 \mu \mathrm{m}$ by $\mathrm{Di}$ Francesco et al. (1994). It is detected (Mannings 1994) at all submm wavelengths; from the $1.3 \mathrm{~mm}$ flux in a FWHM beam of $28^{\prime \prime}$ (Hillenbrand et al. 1992), Natta et al. (1997) derive a circumstellar mass of $0.013 M_{\odot}$. Most of the matter is likely to be in a circumstellar disk, detected at $2.3 \mathrm{~mm}$ with the OVRO interferometer (Mannings \& Sargent 1997). 


\section{$L k H \alpha 218$}
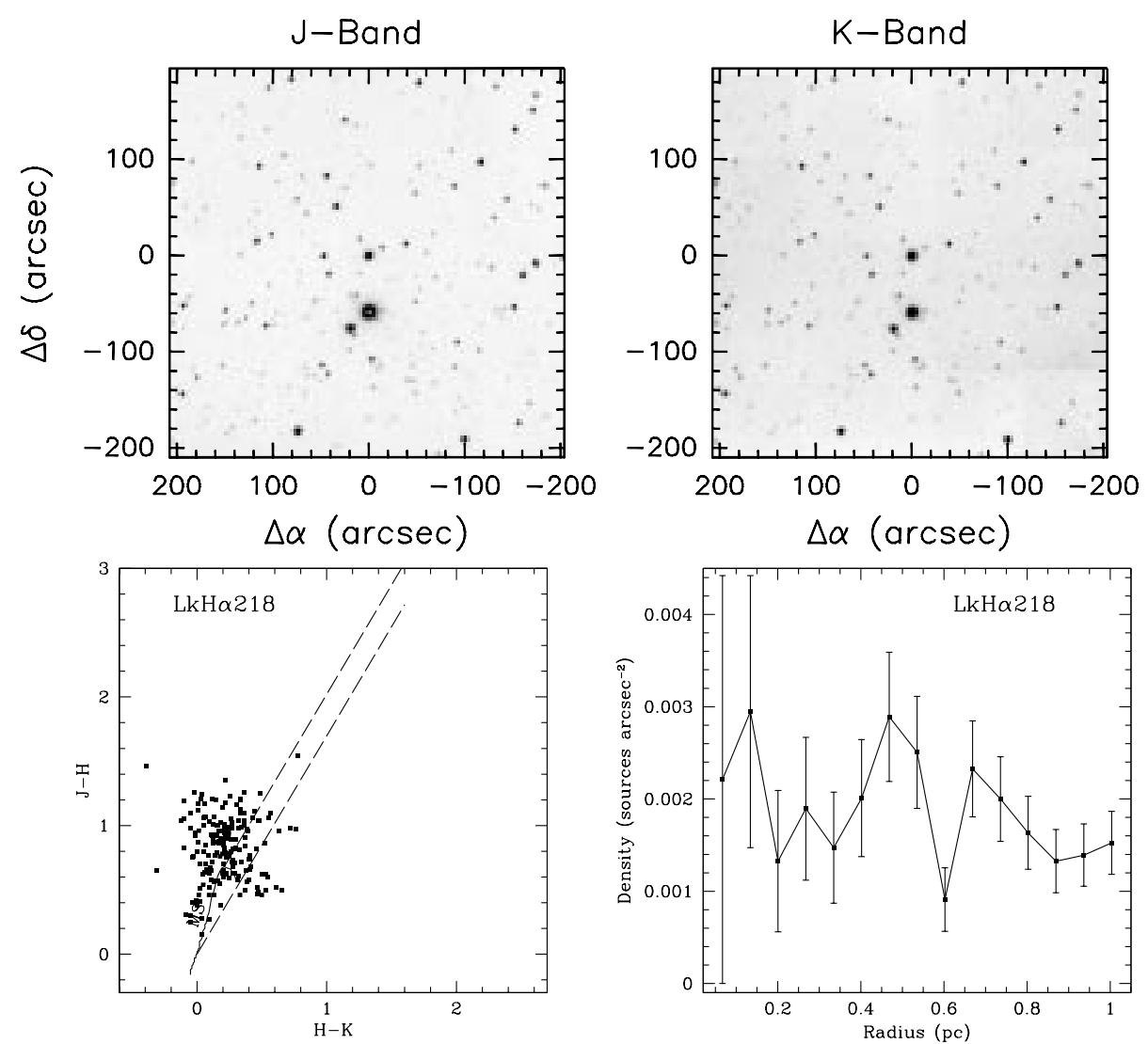

Fig. 31. LkH $\alpha$ 218. Top left: $J$-band image; top right: $K$-band image; bottom left: colour-colour diagram; bottom right: $K$-band source surface density profile

(Fig. 32). AB Aur is an isolated very bright star with an extended halo detected in all our images.

\subsection{VX Cas (HBC 329; IRAS 00286+6142)}

The $V$-magnitude, $(B-V)$ colour and the spectral type (A0) are from Shevchenko et al. (1993). The distance $(760 \mathrm{pc})$ is from Natta et al. (1997).

The star is not detected at $1.3 \mathrm{~mm}$ (Natta et al. 1997; $\left.F_{1.3 \mathrm{~mm}}<6 \mathrm{mJy}\right)$.

(Fig. 33). Rather crowded field, a moderate density enhancement is detected around the Herbig AeBe star within $r \sim 0.3 \mathrm{pc}$.

\subsection{HD 245185 (V1271 Ori; $B D+09^{\circ} 880 ; H B C$ 451; IRAS 05324+0959)}

The $V$-magnitude and $(B-V)$ colour are from Shevchenko et al. (1993). The spectral type (A2) and distance (400 pc) that we assume is that of Hillenbrand et al. (1992).

Mannings (1994) reports a single-dish $1.3 \mathrm{~mm}$ flux of $44 \mathrm{mJy}$ for this source, which corresponds to about
$0.05 M_{\odot}$. The star has also been detected in the $2.7 \mathrm{~mm}$ continuum by Mannings \& Sargent (1997) with the OVRO interferometer, but not in the $\mathrm{CO}(1-0)$ line.

(Fig. 34). In our NIR images the field appears to be very crowded but without a clear density enhancement around the Herbig AeBe star.

\subsection{2. $M W C 480$ (HD 31648; HIP 23143; BD+29 774)}

The $V$-magnitude and the $(B-V)$ colour are from the Hipparcos catalogue (van den Ancker et al. 1998). The distance $(140 \mathrm{pc})$ and the spectral type (A2) are from Mannings et al. (1997).

The Hipparcos parallax give an estimate of the distance consistent with what we assume (131 pc).

Mannings \& Sargent (1997) quote a single-dish $1.3 \mathrm{~mm}$ flux of $360 \mathrm{mJy}$, from which we estimate a mass of circumstellar gas and dust of the order of $0.05 M_{\odot}$. They detect a compact continuum emission at $2.6 \mathrm{~mm}$ and $\mathrm{CO}$ source with the OVRO interferometer, that they interpret as evidence of a circumstellar disk. The presence of a circumstellar rotating gaseous disk is confirmed by the observations of Mannings et al. (1997). 


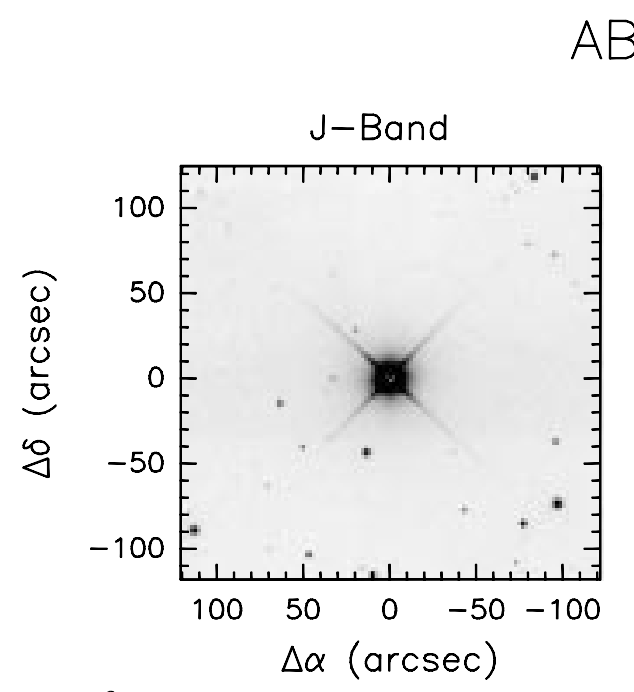

\section{AB Aur}
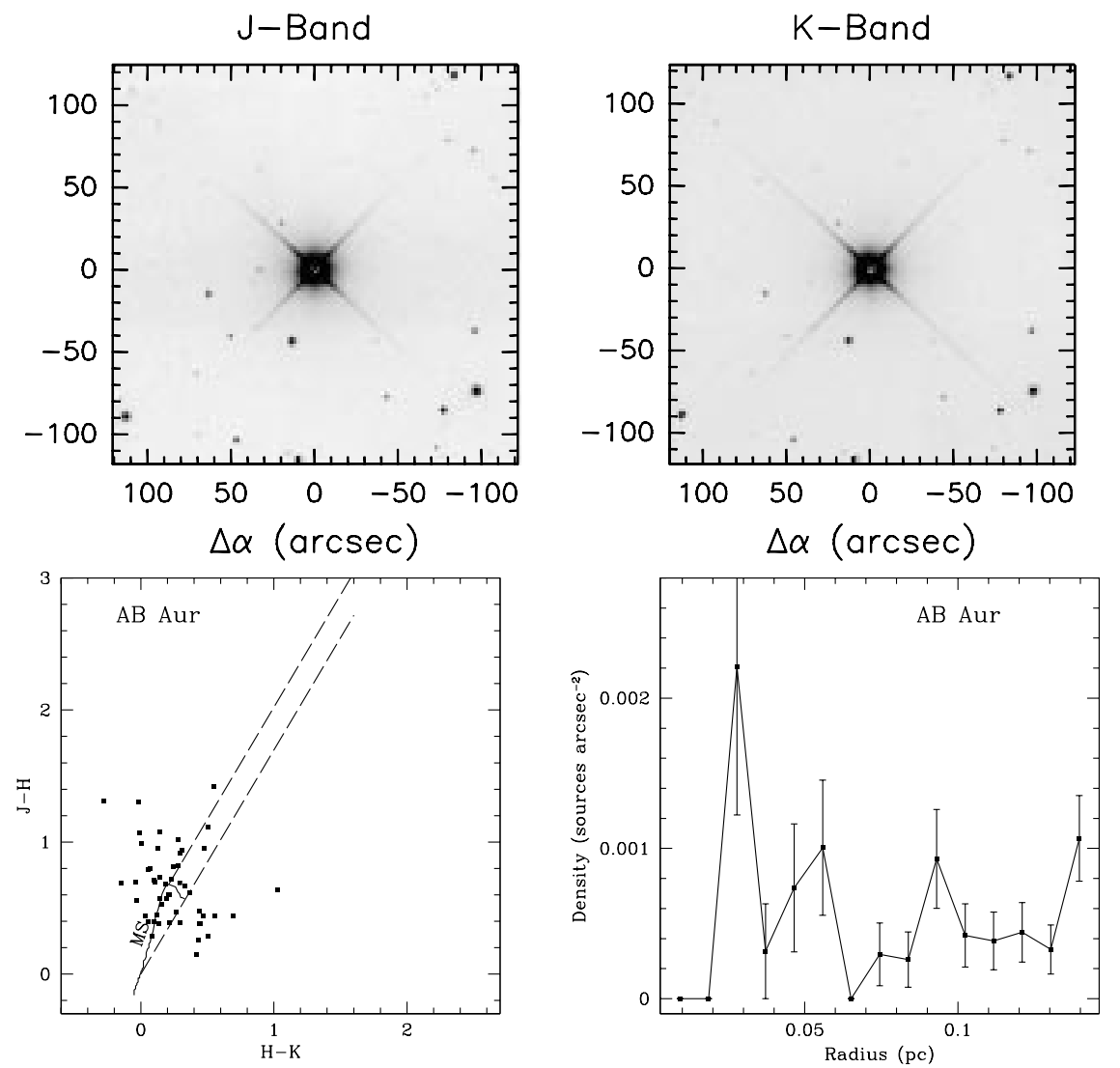

Fig. 32. AB Aur. Top left: $J$-band image; top right: $K$-band image; bottom left: colour-colour diagram; bottom right: $K$-band source surface density profile
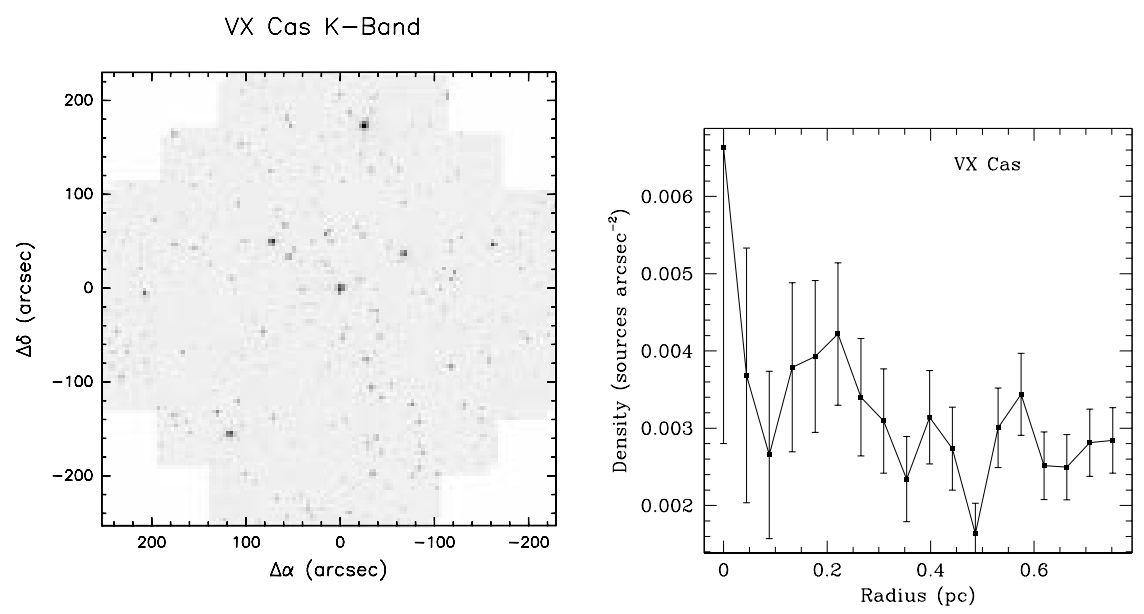

Fig. 33. VX Cas. Left: $K$-band image; right: $K$-band source surface density profile 


\section{HD 245185}
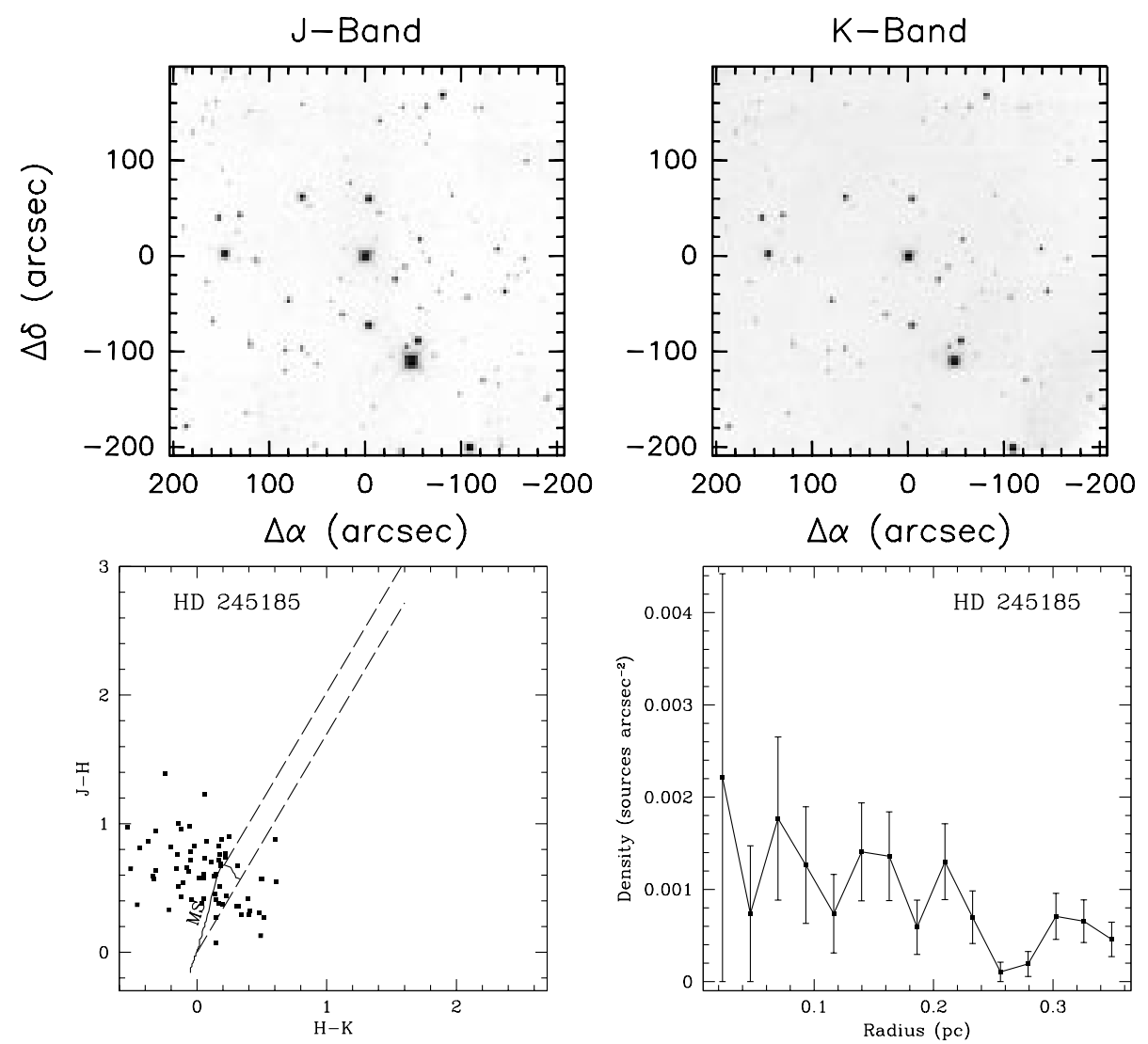

Fig. 34. HD 245185. Top left: $J$-band image; top right: $K$-band image; bottom left: colour-colour diagram; bottom right: $K$-band source surface density profile
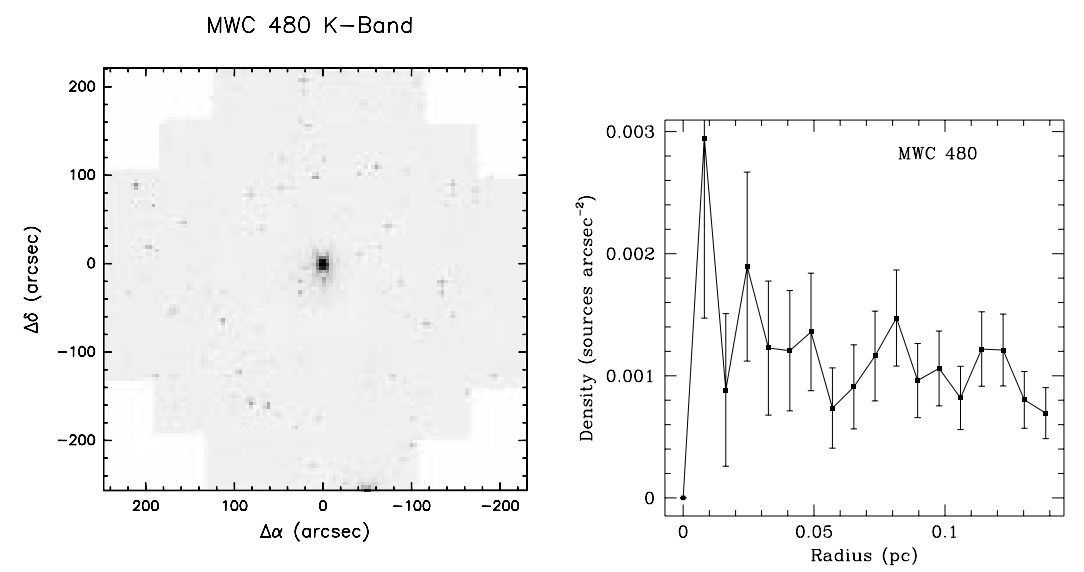

Fig. 35. MWC 480. Left: $K$-band image; right: $K$-band source surface density profile 


\section{UX Ori}
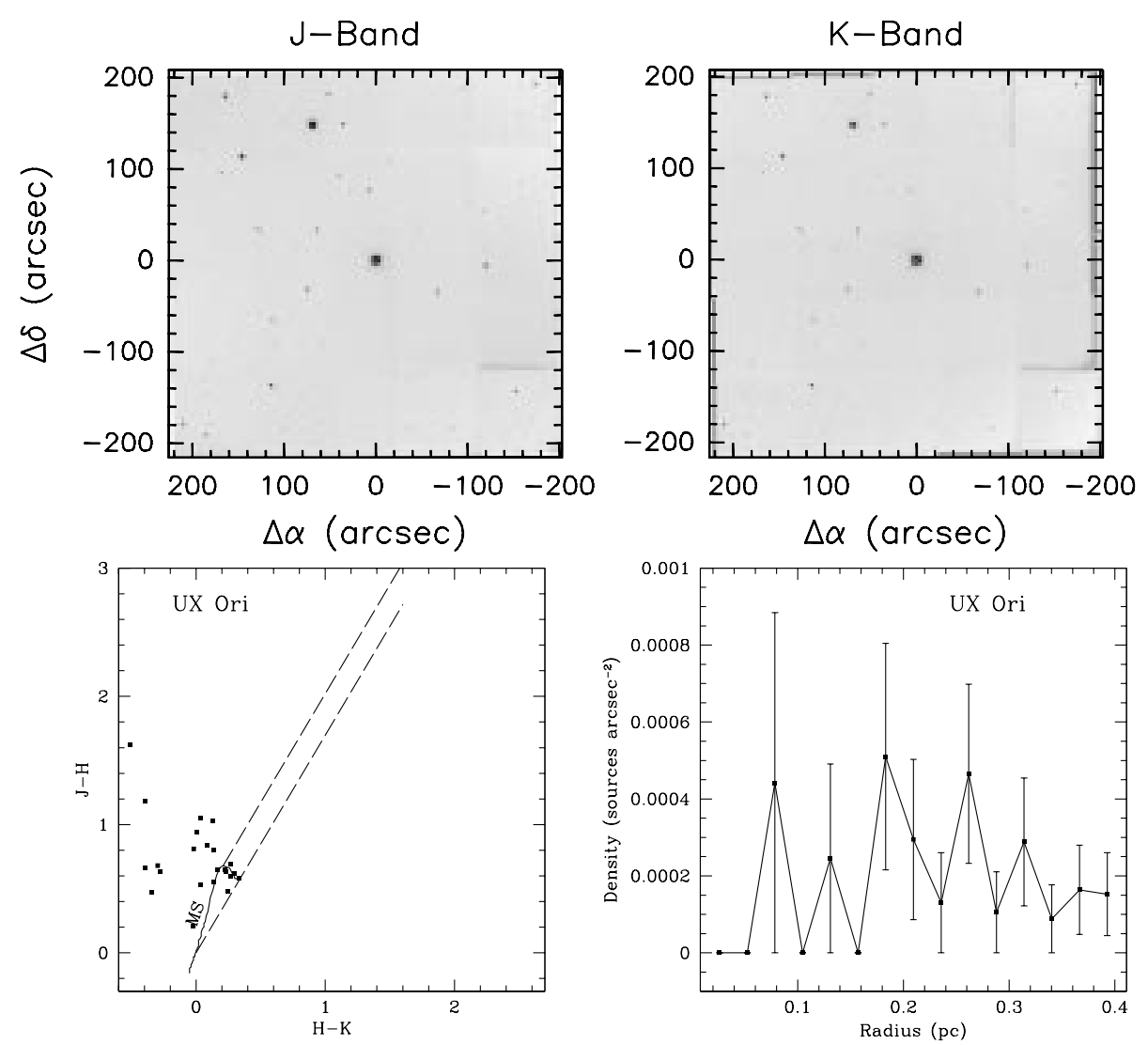

Fig. 36. UX Ori. Top left: $J$-band image; top right: $K$-band image; bottom left: colour-colour diagram; bottom right: $K$-band source surface density profile

(Fig. 35). No clear source surface density enhancement is detected.

\subsection{UX Ori (HD 293782; $B D-04^{\circ} 1029$; IRAS 05020-0351; HBC 430)}

The $V$-magnitude and $(B-V)$ colour are from Shevchenko et al. (1993). The distance (460 pc) and spectral type (A2) are from Hillenbrand et al. (1992).

Hipparcos data (van den Ancker 1998) give $d>130 \mathrm{pc}$, AIIIe, $\log \left(T_{\text {eff }}\right)=3.93 \mathrm{~K}, \log (L)>0.40 L_{\odot}$, which are consistent with what we assume.

The star is detected at $1.3 \mathrm{~mm}$ by Natta et al. (1997), who measure a mass $0.03 M_{\odot}$ in a region of size $0.02 \mathrm{pc}$.

(Fig. 36). Rather empty field, no density enhancement detected around the Herbig AeBe star.

\subsection{T Ori (BD-05 1329; $M W C$ 763; Haro 4-123; NGC 1977 884)}

The $V$-magnitude and $(B-V)$ colour are from Shevchenko et al. (1993). The distance (460 pc) is from Hillenbrand et al. (1992) and spectral type that we adopt (A2) from Hillenbrand (1995); however, Hillenbrand et al. (1992) give B9, whereas Finkenzeller \& Mundt (1984) give A3.

It is known as an eclipsing and spectroscopy binary with a period of 14.3 days (Shevchenko \& Vitrichenko 1994); Hillenbrand (1995) finds a companion at $2.2 \mu \mathrm{m} \mathrm{7.7^{ \prime \prime }}$ away (3500 AU) (confirmed by Leinert et al. 1997), however, due to the high stellar enhancement of the field it is unclear whether it is a real companion; its estimated luminosity is $\sim 2 L_{\odot}$ (typical of a low mass TTauri star).

Henning et al. (1994) measure a $1.3 \mathrm{~mm}$ flux of $88 \mathrm{mJy}$ with a beam of $23^{\prime \prime}$, which corresponds to $0.12 M_{\odot}$ within a radius of 0.025 pc (Natta et al. 1997).

(Fig. 37). Large scale extended emission is clearly detected in all the NIR bands. There seems to be a general tendency of a higher stellar density toward the north-west of the Herbig AeBe star, however no clear density enhancement is detected around it. 


\section{T Ori}
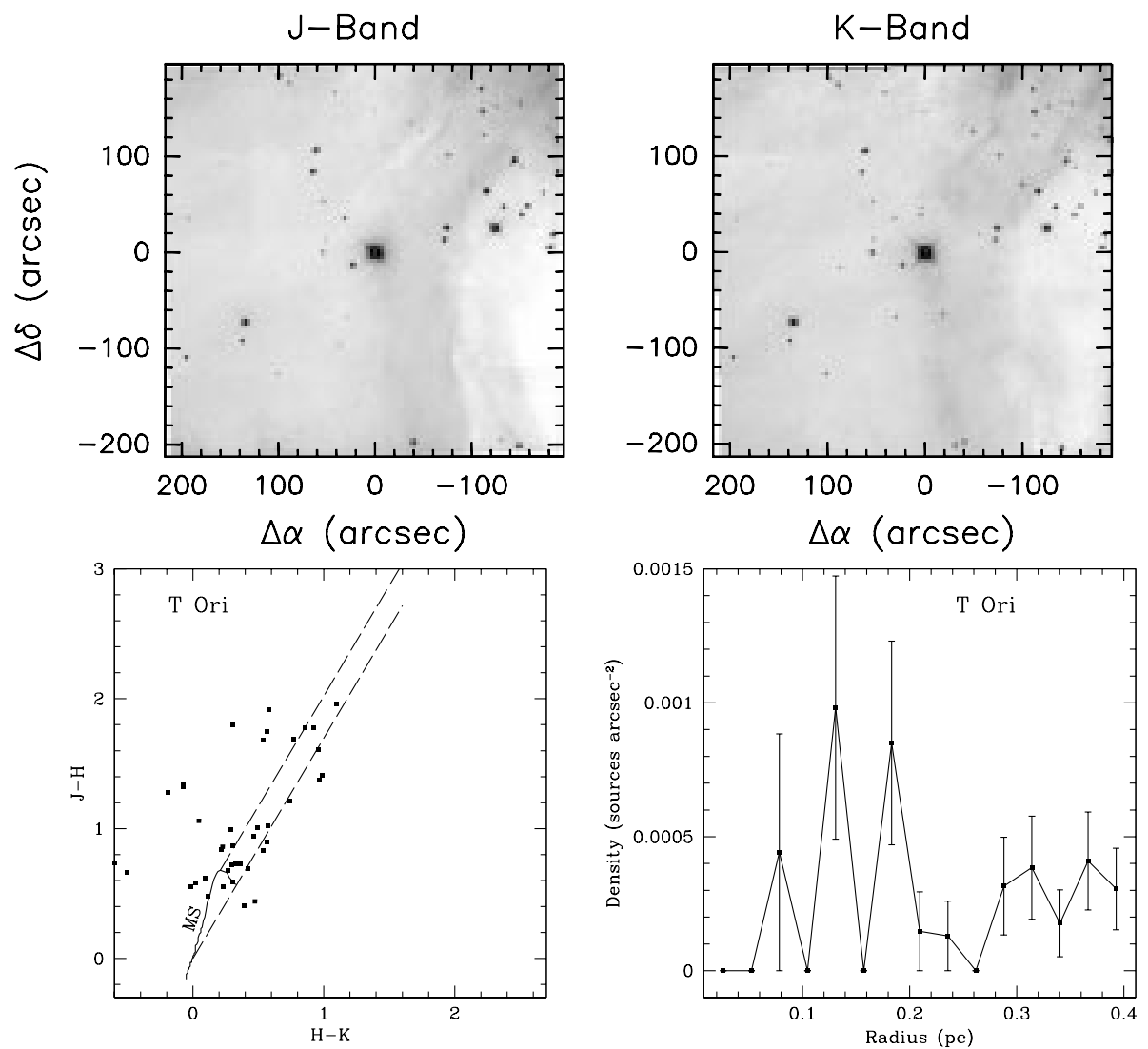

Fig. 37. T Ori. Top left: $J$-band image; top right: $K$-band image; bottom left: colour-colour diagram; bottom right: $K$-band source surface density profile
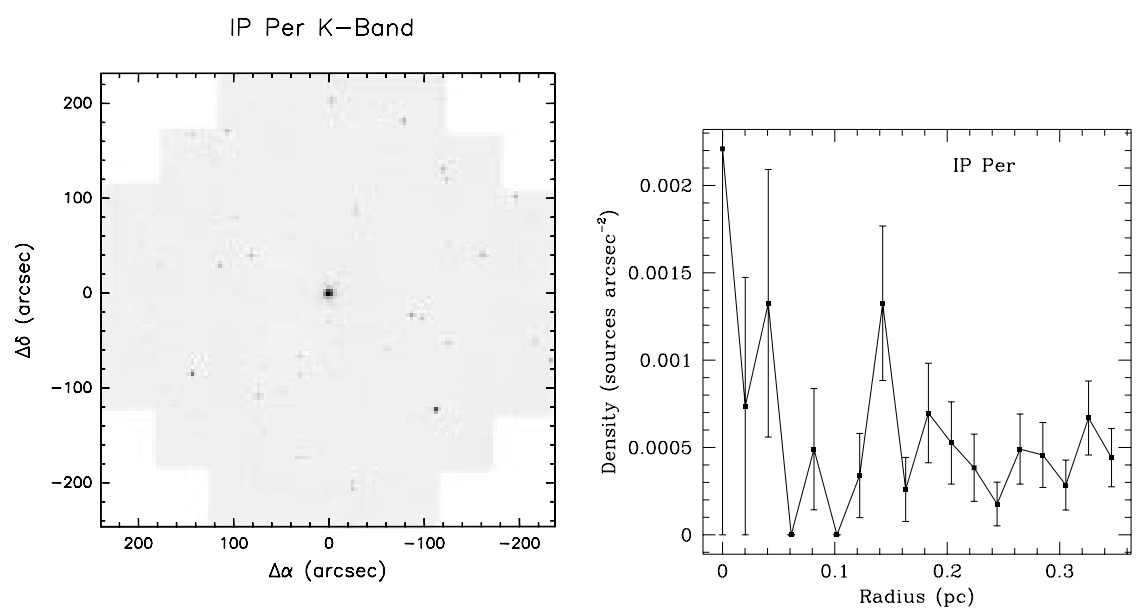

Fig. 38. IP Per. Left: $K$-band image; right: $K$-band source surface density profile 


\section{$L k H \alpha 208$}
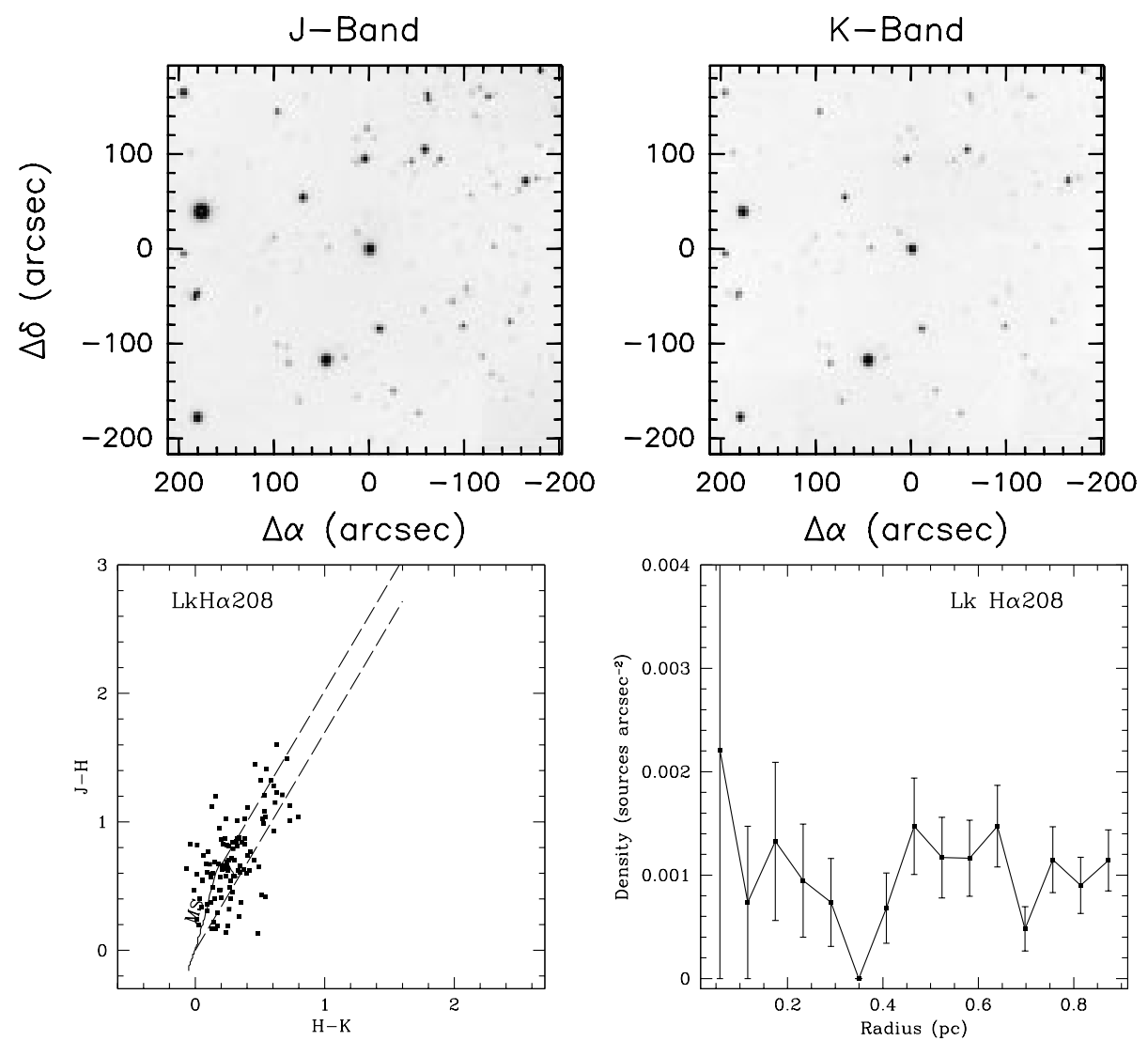

Fig. 39. LkH $\alpha$ 208. Top left: $J$-band image; top right: $K$-band image; bottom left: colour-colour diagram; bottom right: $K$-band source surface density profile

\subsection{IP Per (HD 278937; $H B C$ 348; $B D+32^{\circ} 656$; IRAS 03376+3222)}

The $V$-magnitude, $(B-V)$ colour and spectral type (A3) are from Herbig \& Bell (1988). The distance of 350 pc attributed to this star assumes that it is a member of the Perseus I OB association (Wood et al. 1994).

(Fig. 38). Rather empty field, no stellar group is detected around the Herbig AeBe star.

\subsection{6. $L k H \alpha 208$ (NGC 2163)}

The $V$-magnitude and $(B-V)$ colour are from Shevchenko et al. (1993). Both the distance $(1000 \mathrm{pc})$ and the spectral type (A3) that we adopt are from Hillenbrand et al. (1992).

Leinert et al. (1997) found a comparatively close binary with a separation of $0.115^{\prime \prime}$ (corresponding to $115 \mathrm{AU})$; its luminosity appears high $\left(\sim 100 L_{\odot}\right)$ but rather uncertain.

Millimeter observations (Natta et al. 1997) give an upper limit to the circumstellar mass $<0.03 M_{\odot}$ within 0.025 pc. However, the molecular survey of
Hillenbrand (1995) yields a mass estimate of $M_{\mathrm{cl}}=$ $640 M_{\odot}$ in a region of $\sim 0.8 \mathrm{pc}$ size.

(Fig. 39). The stellar surface density appears to be constant across the whole field. A number of stars show a consistent amount of extinction (as seen from the $(J-H$, $H-K$ ) colour-colour diagram).

\subsection{7. $M W C 758$ (HD 36112; $B D+25^{\circ} 843$; \\ IRAS 05273+2517; HIP 25793)}

The $V$-magnitude and the $(B-V)$ colour are from the Hipparcos catalogue (van den Ancker et al. 1998). The distance and spectral type that we adopt are those reported by Mannings \& Sargent (1997), who give A5 and $D=150 \mathrm{pc}$.

Mannings \& Sargent (1997) quote a single-dish $1.3 \mathrm{~mm}$ flux of $72 \mathrm{mJy}$, which corresponds to $0.01 M_{\odot}$ of circumstellar gas and dust; their OVRO observations also detect compact continuum and $\mathrm{CO}$ emission which is likely to come from a circumstellar disk.

(Fig. 40). A stellar surface density enhancement with $r \sim 0.03 \mathrm{pc}$ is marginally detected. 


\section{MWC 758}
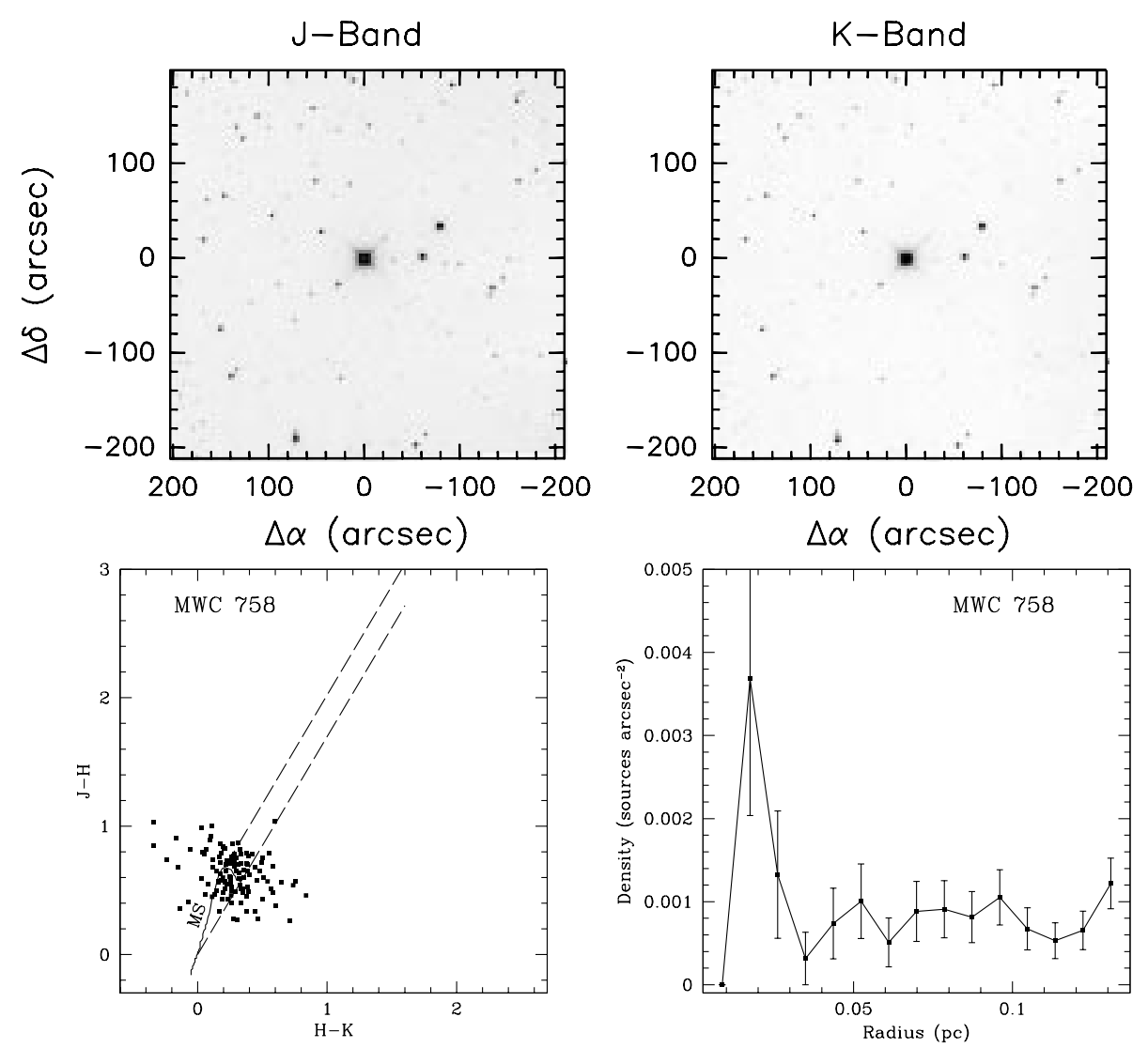

Fig. 40. MWC 758. Top left: $J$-band image; top right: $K$-band image; bottom left: colour-colour diagram; bottom right: $K$-band source surface density profile

\subsection{RR Tau $\left(B D+26^{\circ} 887 a ; H D\right.$ 245906; IRAS 05363+2620; HBC 170; AS 103)}

The $V$-magnitude and $(B-V)$ colour are from Shevchenko et al. (1993). The distance $(800 \mathrm{pc})$ is from Finkenzeller \& Mundt (1984) and the spectral type (A4) from Hillenbrand et al. (1992).

Millimeter continuum observations (Henning et al. 1994) give only an upper limit to the amount of circumstellar matter in the immediate surroundings of the star $\left(<0.03 M_{\odot}\right.$ within a distance of $\left.0.05 \mathrm{pc}\right)$. However, the molecular survey of Hillenbrand (1995) yields a mass estimate of $M_{\mathrm{cl}}=240 M_{\odot}$ in a region of $\sim 1.6 \mathrm{pc}$ size.

(Fig. 41). In our NIR images, the field appears to be rather crowded, with no density enhancement.

\subsection{HK Ori (MWC 497; $M H \alpha$ 265-13)}

The $V$-magnitude and $(B-V)$ colour are from Shevchenko et al. (1993). The distance (460 pc) from Hillenbrand et al. (1992) and spectral type (A4) from Finkenzeller \& Mundt (1984).
$K$-band speckle interferometry (Leinert et al. 1997) reveals a companion at $0.34^{\prime \prime}(156 \mathrm{AU})$, which is probably a low mass young star.

There is no molecular gas left around HK Ori. Fuente et al. (1998) did not detect ${ }^{13} \mathrm{CO}$ and CS emission, while $1.3 \mathrm{~mm}$ continuum observations provide an upper limit to the dust mass of $<0.5 M_{\odot}$ within $0.08 \mathrm{pc}$.

(Fig. 42). Our NIR observations are affected by large photometric uncertainties (see Paper I). No clear density enhancement detected around the star. The colourcolour diagram reveals a probable systematic offset in the photometry.

\subsection{MaC H12 (HBC 1; PP 1)}

The distance $(850 \mathrm{pc})$ and the spectral type (A5) that we adopt are from Cohen \& Kuhi (1976).

Osterloh \& Beckwith (1995) detect the source at $1.3 \mathrm{~mm}$; from their $1.3 \mathrm{~mm}$ flux, we derive a mass of circumstellar matter of $0.2 M_{\odot}$ in a region of $0.04 \mathrm{pc}$ size.

(Fig. 43). Moderately crowded field, a small group of stars is marginally detected within $r \sim 0.15 \mathrm{pc}$ from the Herbig AeBe star. 


\section{RR Tau}
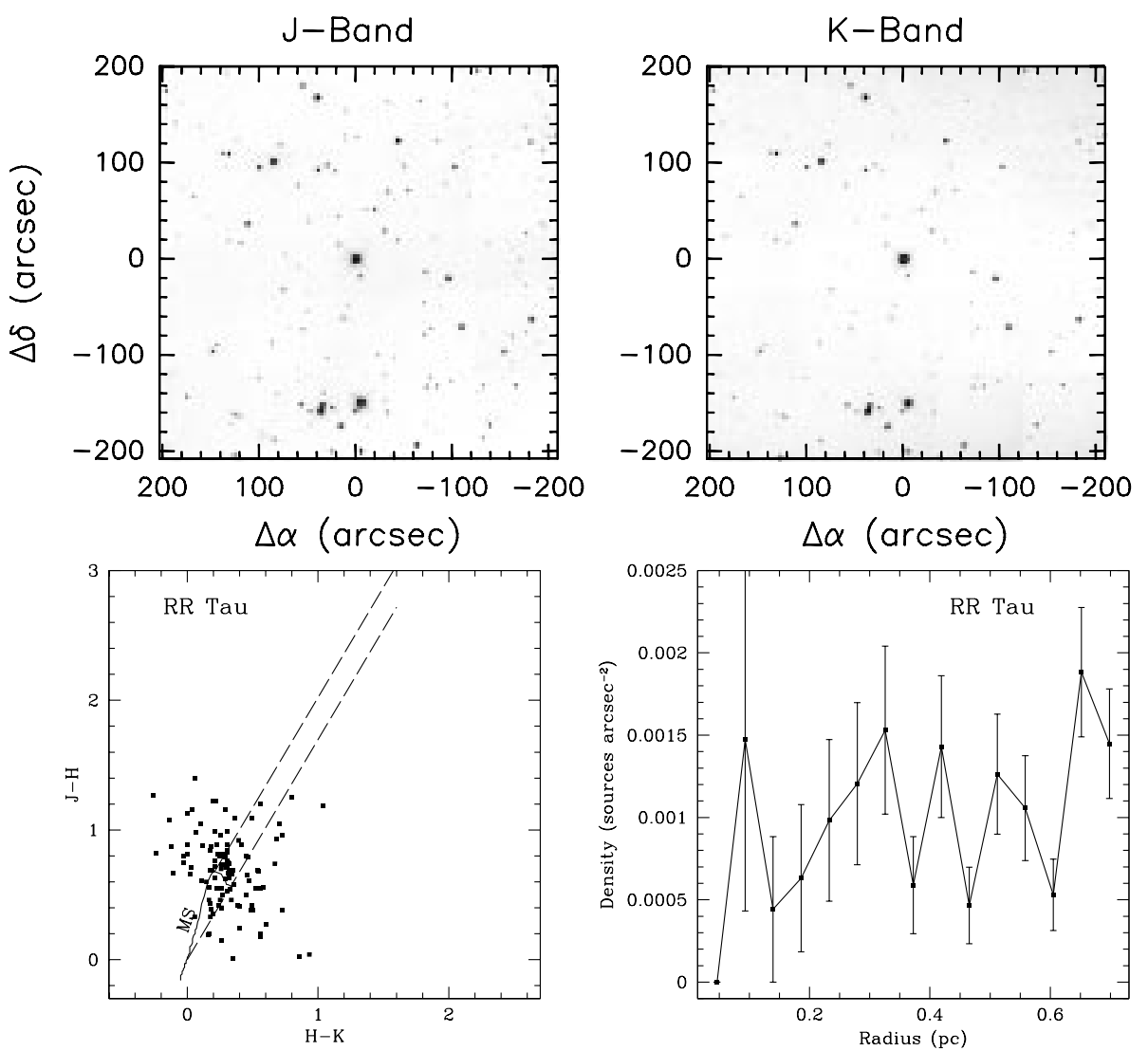

Fig. 41. RR Tau. Top left: $J$-band image; top right: $K$-band image; bottom left: colour-colour diagram; bottom right: $K$-band source surface density profile

\subsection{LkHo 198 (V633 Cas)}

The $V$-magnitude and $(B-V)$ colour that we use are from Shevchenko et al. (1993). The distance $(600 \mathrm{pc})$ is from Chavarria-K. (1985), and the spectral type (A5) from Hillenbrand et al. (1992).

The mid-infrared companion found by Lagage et al. (1993) at $10 \mu \mathrm{m} 6^{\prime \prime}$ north of the optical source is also detected in the NIR (Li et al. 1994; Leinert et al. 1997). The projected separation of the binary is $3300 \mathrm{AU}$; the estimated luminosity of the companion is $\sim 100 L_{\odot}$, thus it could be the third Herbig AeBe star in the system.

Natta et al. (1993), from 50 and $100 \mu \mathrm{m}$ observations, infer the existence of a dusty envelope surrounding the star, with a mass of about $45 M_{\odot}$ within 0.5 pc. Millimeter observations at $1.3 \mathrm{~mm}$ (Hillenbrand et al. 1992) and $2.7 \mathrm{~mm}$ (Di Francesco et al. 1997) reveal an extended source; the mass of dust and gas in a region of size $0.08 \mathrm{pc}$ is about $1 M_{\odot}$. The molecular survey of Hillenbrand (1995) yields a mass estimate of $M_{\mathrm{cl}}=$ $373 M_{\odot}$ in a region of 0.4 pc size. Higher resolution observations by Fuente et al. (1998) show that on a scale of $0.08 \mathrm{pc}$ the amount of gas and dust is reduced to $\sim 5 M_{\odot}$.
(Fig. 44). The two bright Herbig AeBe stars LkH $\alpha 198$ and V 376 Cas are embedded in a diffuse nebulosity detected in all the three NIR bands. Faint sources close to the bright stars are difficult to detect within the extended emission. The $K$-band source surface density increases away from the central stars, suggesting that either the diffuse emission or the increased extinction due to a compact molecular clump localized around the Herbig AeBe stars prevent the detection of background stars and, possibly, faint companions.

Support to this possibility comes from the submm continuum observations of Sandell \& Weintraub (1994) who found an embedded source at the center of a molecular outflow. $2.7 \mathrm{~mm}$ observations of Di Francesco et al. (1997) indicate that this source is quite extended and may thus provide a substantial amount of spatially extended extinction.

\subsection{Elias 1 (V892 Tau)}

The $V$-magnitude and $(B-V)$ colour are from Berrilli et al. (1992), the distance (160 pc) and spectral type (A6) are from Hillenbrand (1995). 


\section{HK Ori}
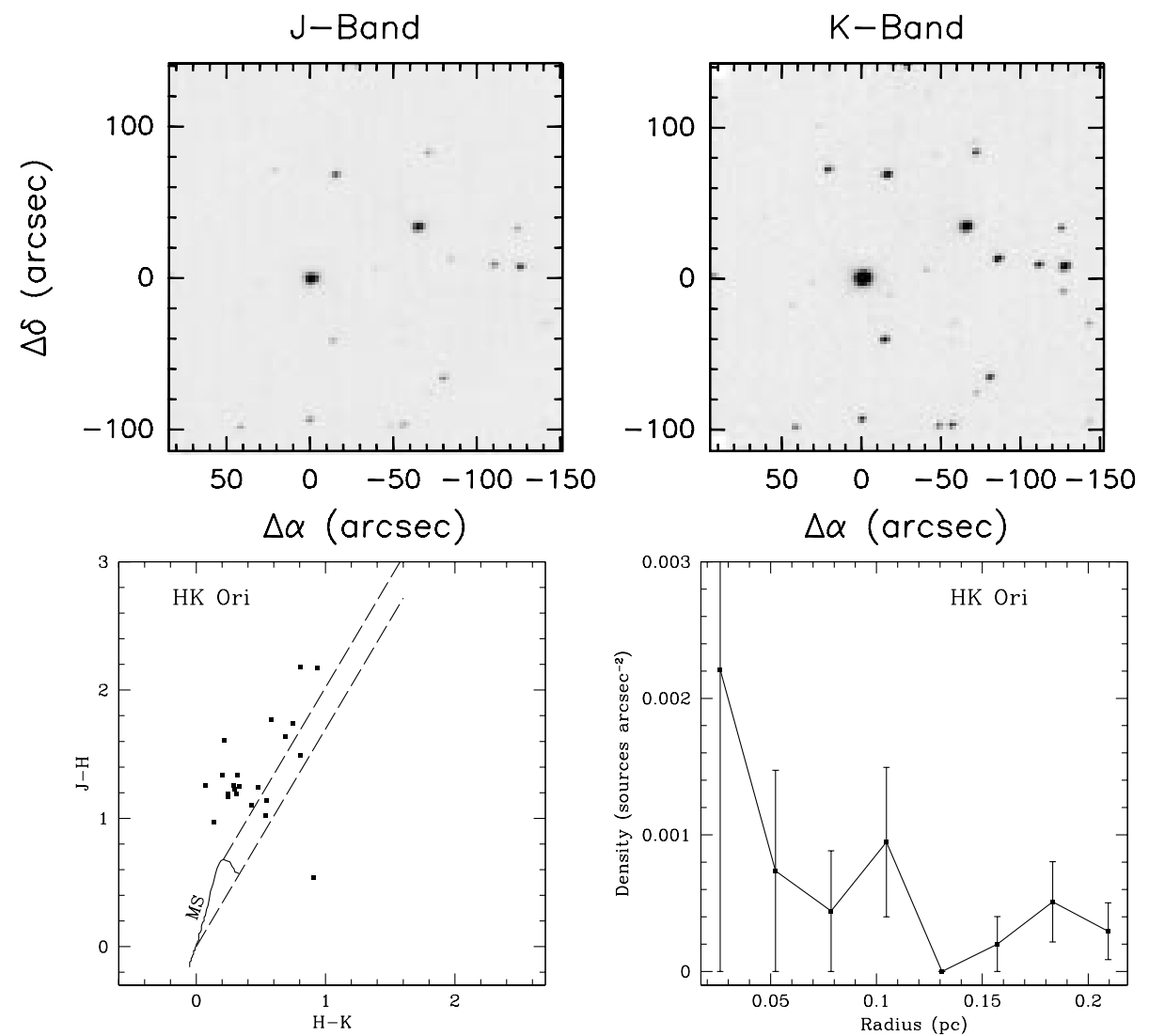

Fig. 42. HK Ori. Top left: $J$-band image; top right: $K$-band image; bottom left: colour-colour diagram; bottom right: $K$-band source surface density profile
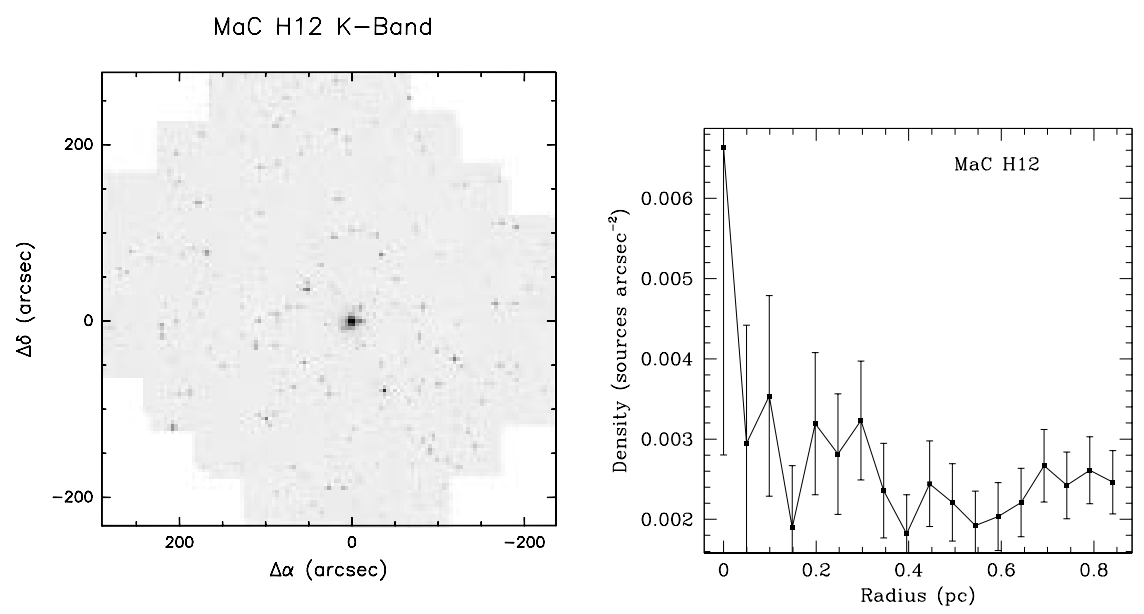

Fig. 43. MaC H12. Left: $K$-band image; right: $K$-band source surface density profile 


\section{LkH $\alpha 198$}
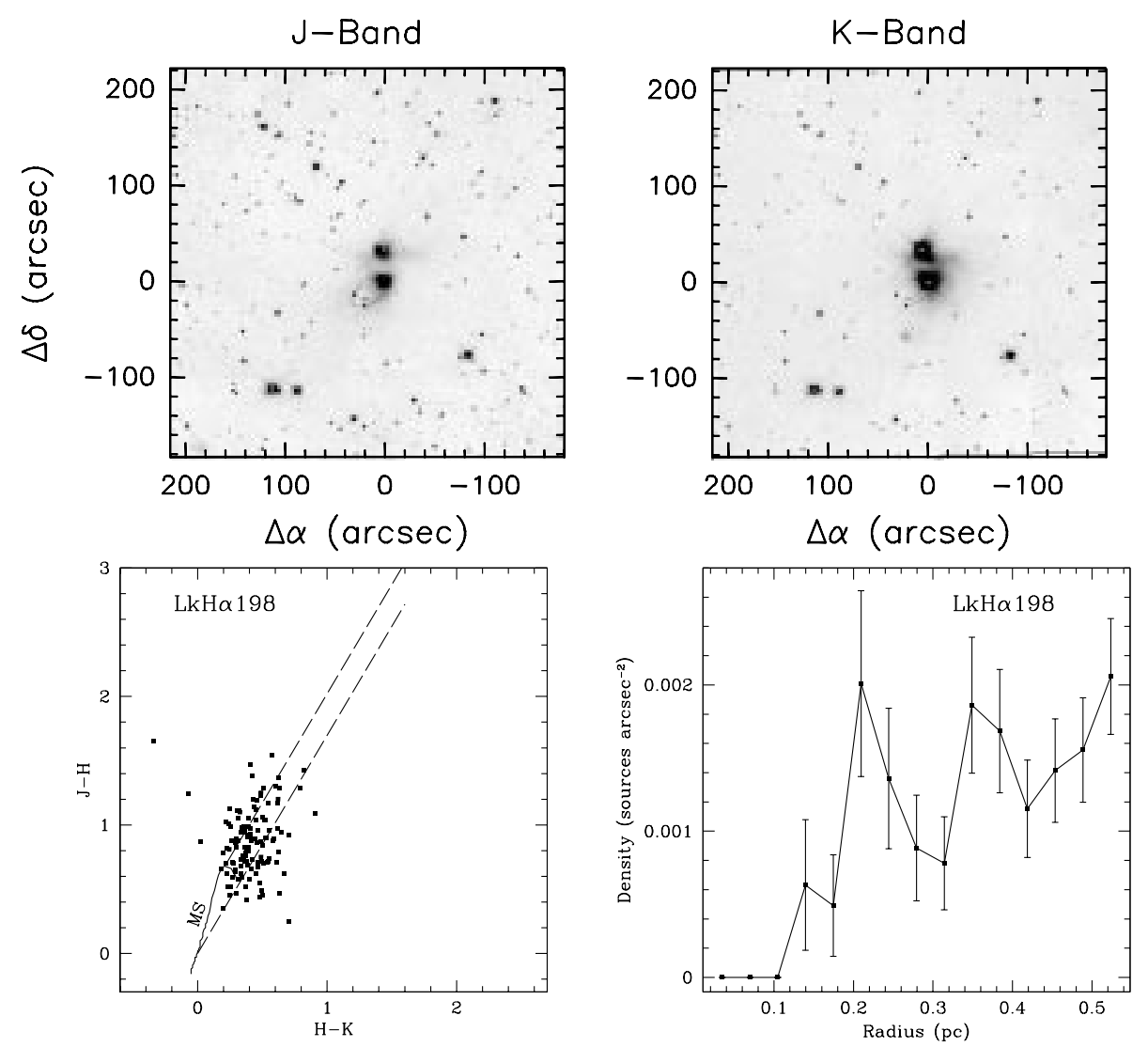

Fig. 44. LkH $\alpha$ 198. Top left: $J$-band image; top right: $K$-band image; bottom left: colour-colour diagram; bottom right: $K$-band source surface density profile

Pirzkal et al. (1997) NIR imaging reveals a companion at $3.72^{\prime \prime}$ (595 AU) with a $K$ mag difference of 4.2 between primary and companion. The companion is also revealed in the speckle interferometry survey for binaries among Herbig AeBe stars of Leinert et al. (1997); they give the IR companion position $4^{\prime \prime}(570 \mathrm{AU})$ to the NE of the target star, fainter by $4-5$ mag; its estimated luminosity is $0.4 L_{\odot}$. It is also detected in the radio continuum survey of Skinner et al. (1993), who agree that it is probably a low mass pre-main sequence star.

Hillenbrand et al. (1992) measure a $1.3 \mathrm{~mm}$ flux of 490 mJy with a beam FWHM of $28^{\prime \prime}$, which corresponds approximately to $0.08 M_{\odot}$ of dust and gas in a region of 0.02 pc size. Di Francesco et al. (1997) detect the star at $2.7 \mathrm{~mm}$ with the $\mathrm{PdB}$ interferometer within $1^{\prime \prime}$ of the optical position.

(Fig. 45). Our NIR images show an almost empty field, with no stellar density enhancement detected. In our images the faint companion is completely hidden by the luminosity of the Herbig AeBe star.

\subsection{BF Ori $\left(B D-06^{\circ} 06\right.$ 1259; $H B C 169$; IRAS 05348-0636; Haro 4-229)}

The $V$-magnitude and $(B-V)$ colour are from Shevchenko et al. (1993). The distance (460 pc) is from Hillenbrand et al. (1992) and the spectral type (A7) from Hillenbrand (1995); however, Hillenbrand et al. (1992) give F2, whereas Finkenzeller \& Mundt (1984) give A/F. We decided to adopt $\mathrm{A} 7$ which represent a mean value of the various determinations.

Hipparcos data (van den Ancker 1998) give: $\pi=$ -0.7 mas, $d>210$ pc, A5-6IIIe, $\log \left(T_{\text {eff }}\right)=$ $3.90 \mathrm{~K}, \log (L)>0.56 L_{\odot}$, consistent with our assumptions.

BF Ori is detected at $1.3 \mathrm{~mm}$ by Natta et al. (1997), who estimate a total mass of $0.008 M_{\odot}$ in a region of 0.05 pc size.

(Fig. 46). No clear density enhancement is detected, even though the brightest sources are located near the Herbig AeBe star. 


\section{Elias 1}
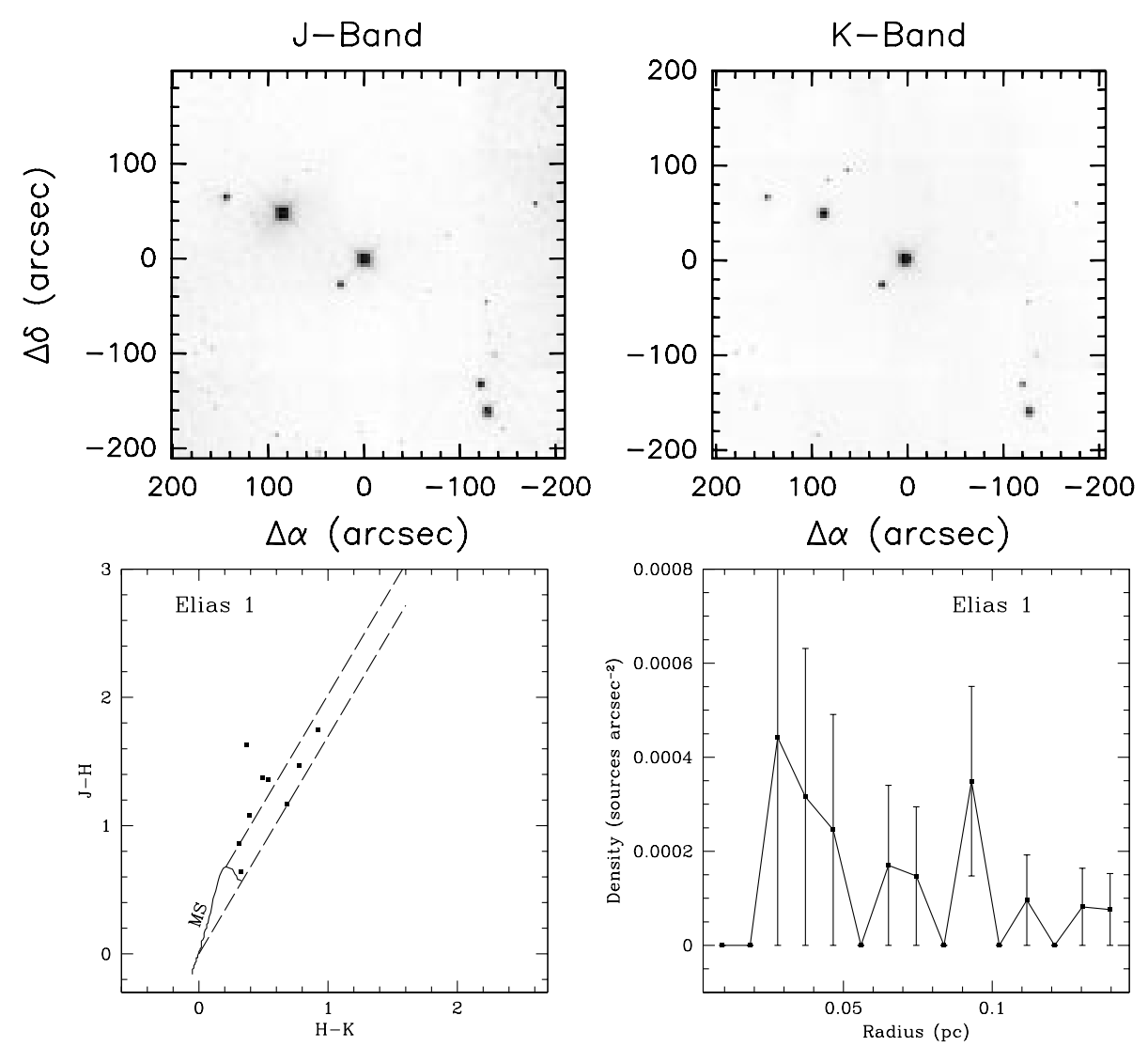

Fig. 45. Elias 1. Top left: $J$-band image; top right: $K$-band image; bottom left: colour-colour diagram; bottom right: $K$-band source surface density profile

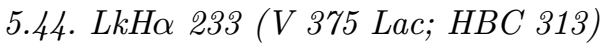

The $V$-magnitude and $(B-V)$ colour are from Shevchenko et al. (1993). The distance $(880 \mathrm{pc})$ and the spectral type (A7) are from Finkenzeller \& Mundt (1984).

The star is not detected at $1.3 \mathrm{~mm}(<40 \mathrm{mJy}$; Hillenbrand et al. 1992), which results into an estimate of the circumstellar mass in a region of $0.12 \mathrm{pc}$ size of $<0.2 M_{\odot}$. The molecular survey of Hillenbrand (1995) yields a mass estimate of $M_{\mathrm{cl}}=250 M_{\odot}$ in a region of $\sim 1.0$ pc size. High resolution observations by Fuente et al. (1998) show that on a scale of $0.08 \mathrm{pc}$ the amount of gas and dust is reduced to $\sim 3.5 M_{\odot}$.

(Fig. 47). Our $K$-band image show a low source surface density field, without a central enhancement. This result is consistent with what has been found by Hillenbrand (1995) in her search for young groups around Herbig AeBe stars.

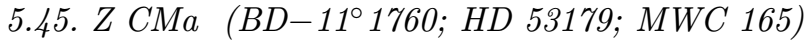

The distance (1150 pc) is from Hillenbrand et al. (1992) and spectral type F5. The spectral type classification is very uncertain and the values quoted in the literature span the range A0-F5. It is not wise to determine luminosity and age from the $V$-magnitude and the $(B-V)$ colour.

(Fig. 49). In our images the bright star is surrounded by a strong diffuse emission. No density peak is detected around the star. The photometry is affected by the systematic uncertainties described in Paper I and an offset in the $H$ band magnitudes is evident in the colour-colour diagram.

\section{Summary}

With the aim of searching for young star clusters around intermediate mass stars, we have selected a sample of 45 Herbig AeBe stars, 43 of which cover almost uniformly the spectral range from O9 to A7. From data existing in the literature we have selected a set of stellar parameters (spectral type, distance from the Sun, $V$-magnitude and $B-V$ colour), from which we have calculated effective temperature and bolometric luminosity for each star. Using the theoretical PMS evolutionary tracks of Palla \& Stahler (1993), we have evaluated the age of almost all the stars with spectral type later than B5. 

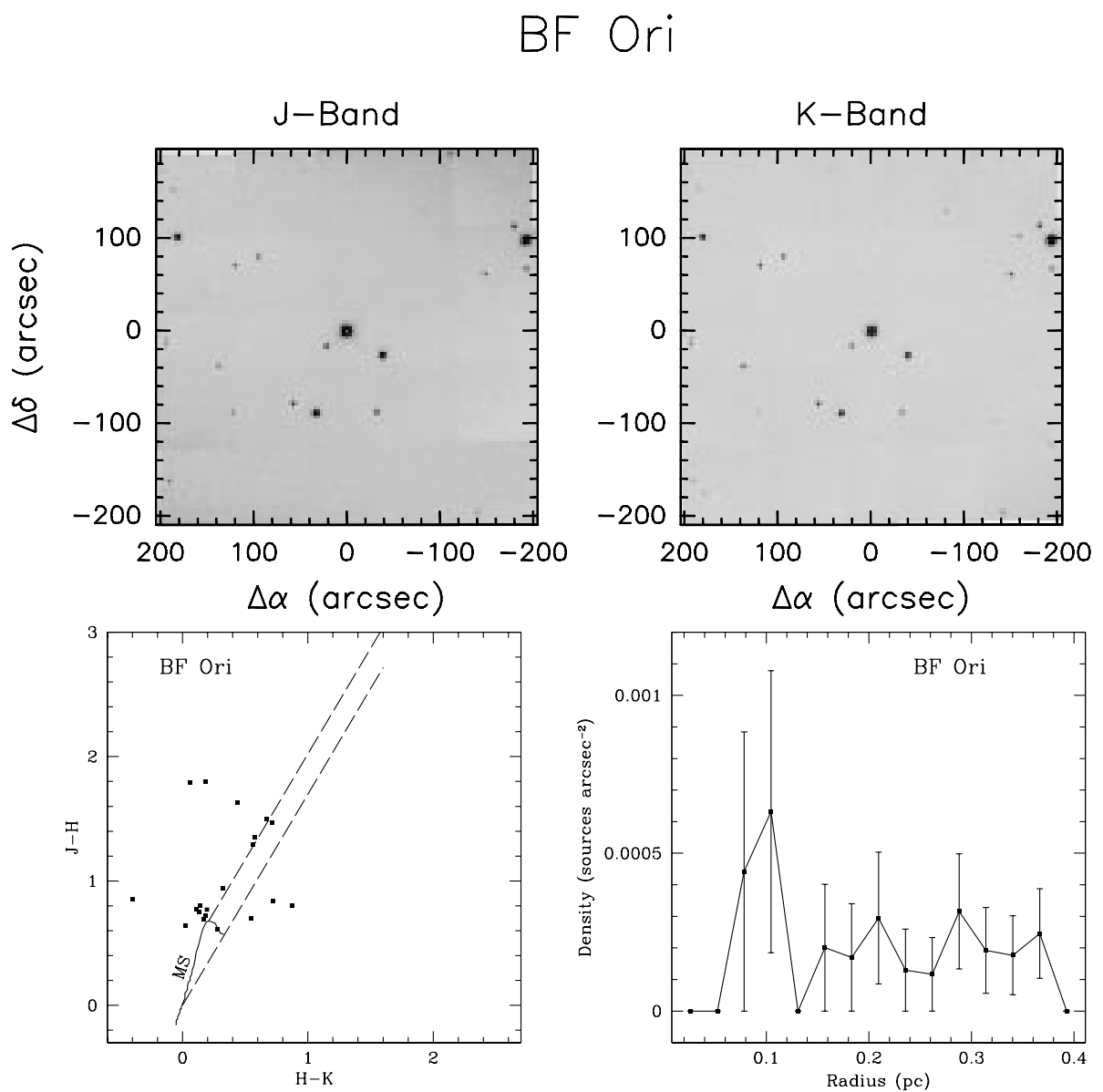

Fig. 46. BF Ori. Top left: $J$-band image; top right: $K$-band image; bottom left: colour-colour diagram; bottom right: $K$-band source surface density profile
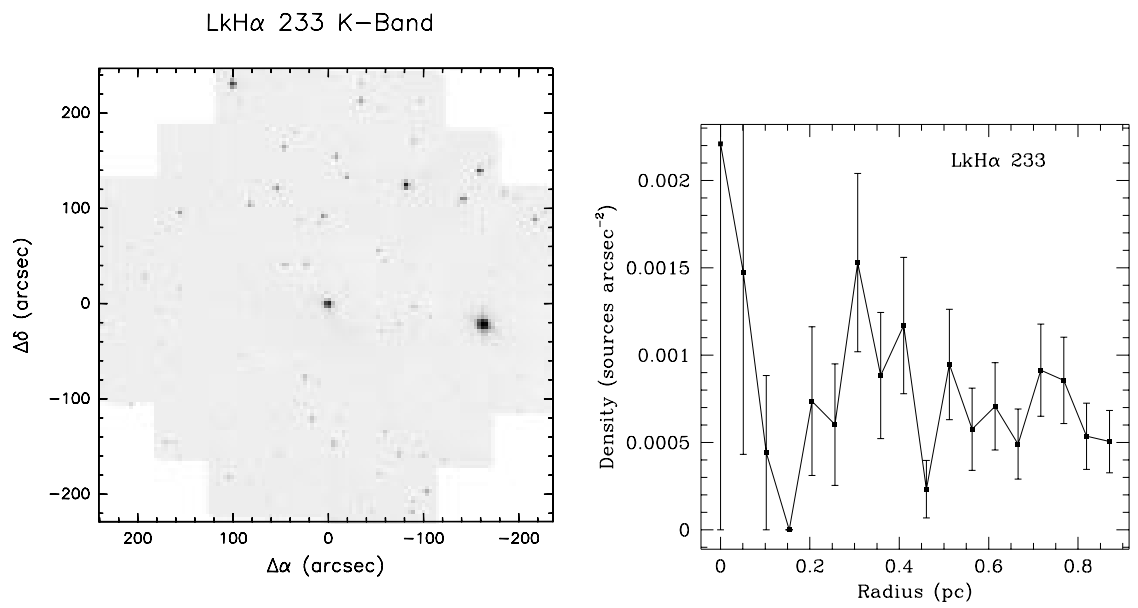

Fig. 47. LkH $\alpha$ 233. Left: $K$-band image; right: $K$-band source surface density profile 


\section{Z $\mathrm{CMa}$}
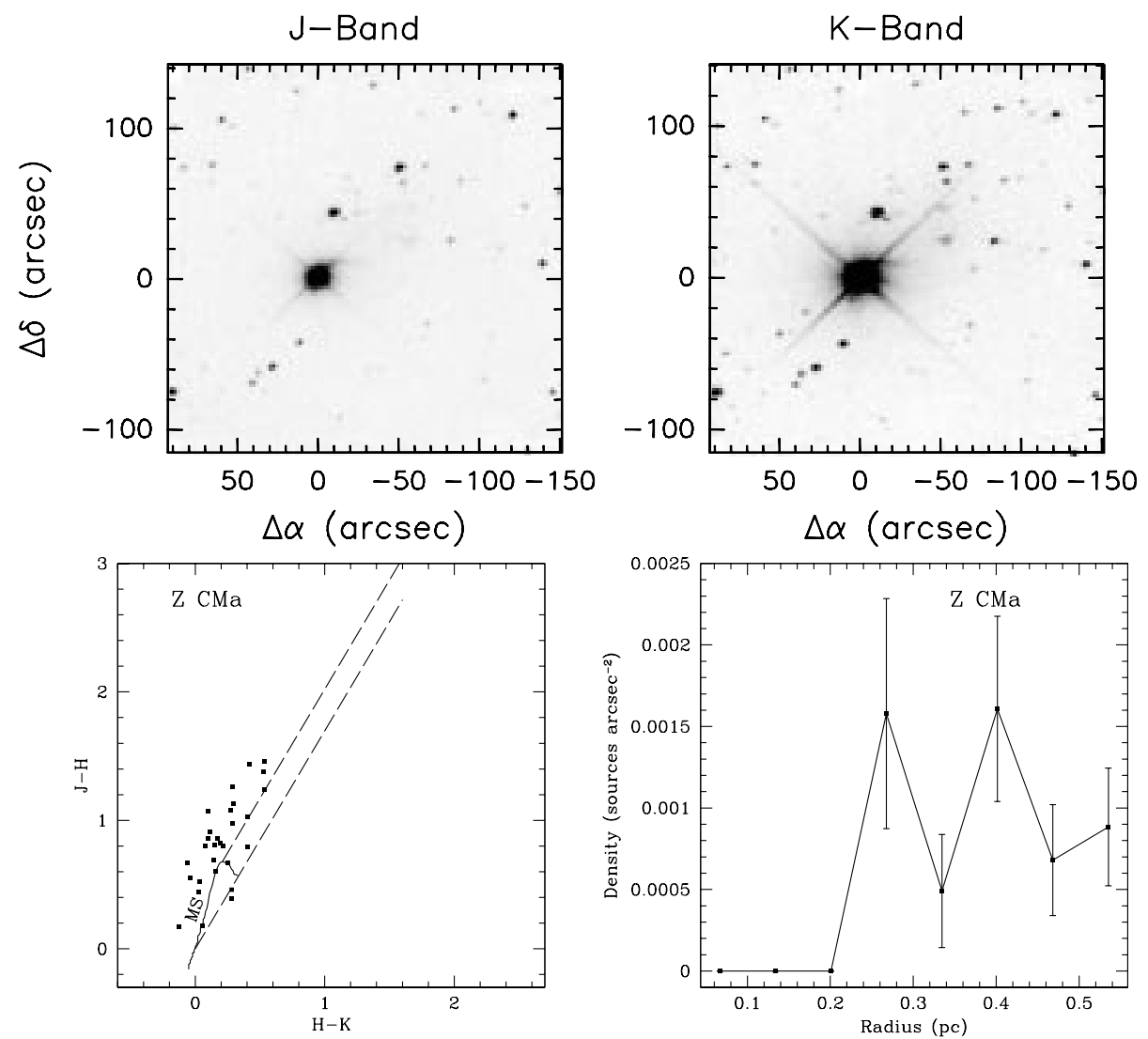

Fig. 48. Z CMa. Top left: $J$-band image; top right: $K$-band image; bottom left: colour-colour diagram; bottom right: $K$-band source surface density profile
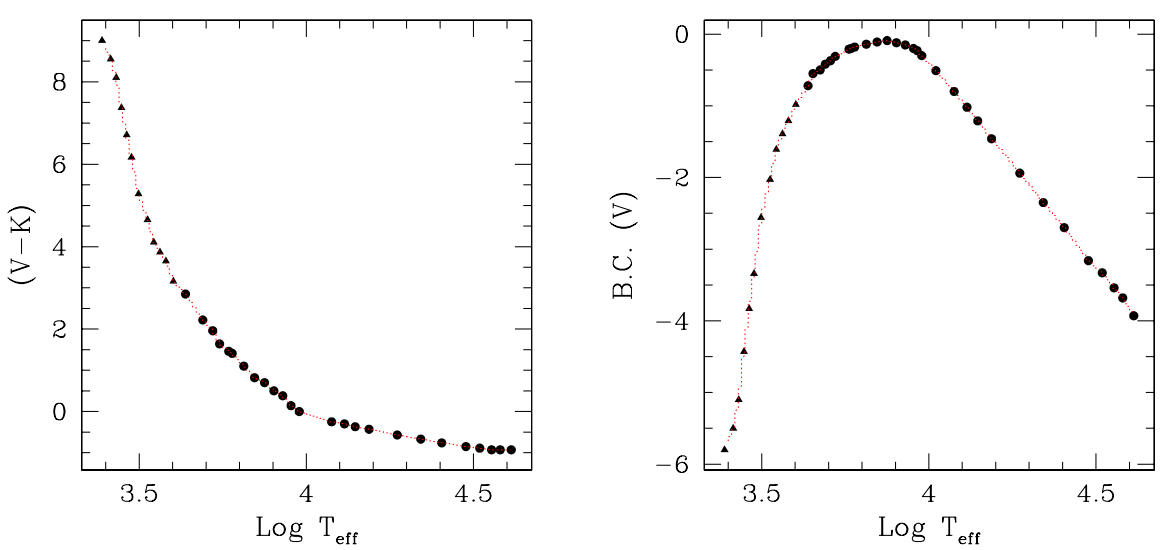

Fig. 49. Adopted $B C_{V}$ vs. $T_{\text {eff }}$ and $(V-K)$ vs. $T_{\text {eff }}$ relations, top and bottom panel respectively. Filled triangles are from the M-dwarfs calibration of Bessel (1991), filled circles are a compilation from the various references given in the text, the dotted line is the linear interpolation that we have used 


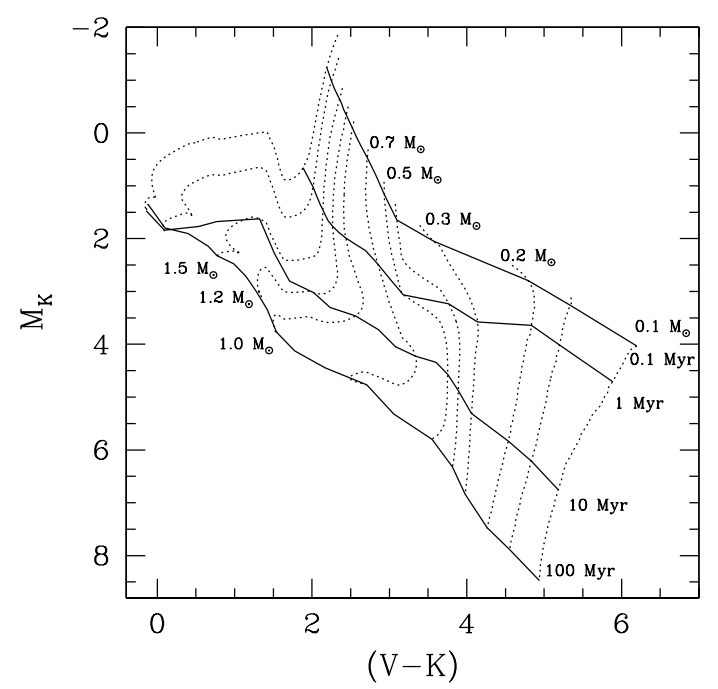

Fig. 50. Colour-magnitude diagram with the D'Antona \& Mazzitelli (1994) tracks. The dotted lines show the evolutionary tracks for $0.1,0.15,0.2,0.3,0.4,0.5,0.7,1.0,1.2,1.5,2.0$, and $2.5 M_{\odot}$, respectively. Continuous lines show the isochrones at $0.1,1$, 10 , and $100 \mathrm{Myr}$
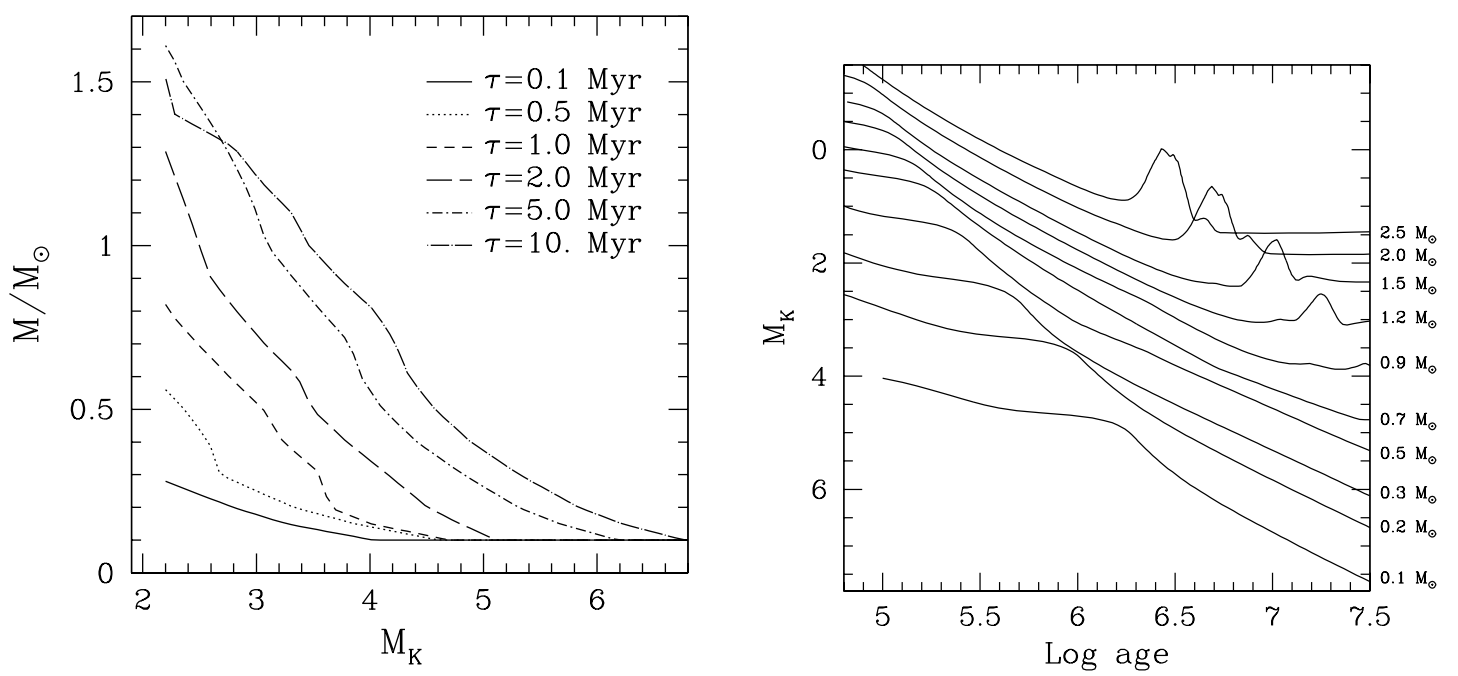

Fig. 51. Top panel: absolute $K$ magnitude as a function of time for $M_{\star} / M_{\odot}=2.5,2.0,1.5,1.2,0.9,0.7,0.5,0.3,0.2,0.1$, respectively (from bottom to top); bottom panel: $M_{\star}$ versus $M_{K}$ for isochrones in the interval $0.1-10$ Myr. Given the $K$ absolute magnitude and the age of a star it is possible to derive the corresponding mass, using the appropriate isochrone

The field around each star has been imaged in the nearinfrared with a moderately large field of view and sensitivity. In 22 fields we found a group of stars likely associated with the Herbig AeBe star and determined the two richness indicators $\mathcal{N}_{K}$ and $I_{\mathrm{C}}$, as well as the radius of the group. The typical size of the detected groups is $\sim 0.2 \mathrm{pc}$. This value is in agreement with that found by other authors in various young stellar clusters (Hillenbrand 1995; Carpenter et al. 1997). It is remarkable that stellar groups with a few to several hundred members share similar sizes, which in turn correspond to the typical sizes of dense cores in molecular clouds.
The observations presented in this paper constitute the dataset for our study of the clustering around Herbig AeBe stars (Paper I; Testi et al. 1998).

Acknowledgements. We thank the TIRGO and ARNICA staff, especially Filippo Mannucci, for nice scheduling and service observing. LT would like to thank the NOT staff for help and hospitality. Special thanks are due to Colin Aspin, Leslie Hunt, Amanda Kaas and Ruggero Stanga who made the NOT run a very pleasent and fruitfull one. The NOT observing run has been partially supported by the Dipartimento di Astronomia e Scienza dello Spazio of the Università di Firenze. This work was partly supported by ASI grant ARS96-66 and CNR grant 97.00018.CT02 to the Osservatorio di Arcetri. Support from C.N.R.-N.A.T.O. Advanced Fellowship 
program and from NASA's Origins of Solar Systems program (through grant NAGW-4030) is gratefully aknowledged. This search has made use of the Simbad database, operated at CDS, Strasbourg, France.

\section{Appendix A}

\section{Derivation of absolute magnitudes and colour indexes from theoretical evolutionary tracks}

In this Appendix we will briefly describe the method that we have used to transform the $K$-completeness absolute magnitude given in Table 1 into an estimate of the lowest mass detectable in each field, the so-called minimum mass. The discussion follows closely the method described in Meyer (1996).

Firstly, we need to convert the PMS evolutionary tracks of D'Antona \& Mazzitelli (1994) from the theoretical HR diagram $\log L_{\star}, \log T_{\text {eff }}$ into the observable one $M_{K},(V-K)$, where $M_{K}$ is the absolute $K$ magnitude. We have used the calibrations of SchmidtKaler (1981), Bessel (1979, 1991), Bessel \& Bret (1988) and Koornneef (1983) for dwarf stars to compile tables of the bolometric corrections at $V$ band $\left(B C_{V}\right)$ and the $(V-K)$ colour index as a function of the effective temperature $\left(T_{\text {eff }}\right)$. These relations are shown in Fig. 48. For each value of $L_{\star}$ and $T_{\text {eff }}$, we can then compute $M_{K}$ as:

$$
\begin{aligned}
M_{K}= & 4.725-2.5 \log \left(L_{\star} / L_{\odot}\right) \\
& -B C_{V}\left(T_{\text {eff }}\right)-(V-K)\left(T_{\text {eff }}\right)
\end{aligned}
$$

where 4.725 is the assumed absolute $V$ magnitude of the Sun. The colour-magnitude $\left(V-K, M_{K}\right)$ diagram resulting from the transformation of the D'Antona \& Mazzitelli (1994) "CM Alexander" (their Fig. 3) tracks is shown in Fig. 50.

We can now derive for each stellar mass the run of the $K$ absolute magnitude with time. This is shown in the upper panel of Fig. 51 for masses in the interval $2.5-0.1 M_{\odot}$. The peak in $M_{K}$ that appears for the more massive stars at $\log ($ age $) \sim 6.2-7.4$ is due to the transition from the convective to the radiative section of the evolutionary tracks (cf. Fig. 50 and Fig. 3 of D'Antona \& Mazzitelli 1994). We can see that in the range of ages $(t<10 \mathrm{Myr})$ and minimum masses considered in this paper (see Tables 1 and 2), the $M_{K}$ of a star of a given mass is a monotonically increasing function of time. For this reason, given a $K$ absolute completness magnitude, the minimum mass detectable is a function of time: as the age of the cluster increases we loose sensitivity on the lowest mass members. In graphical form this is presented in the lower panel of Fig. 51, where the masses corresponding to $M_{K}$ are plotted for isochrones between 0.1 to $10 \mathrm{Myr}$.

We have used this last figure to derive the minimum mass in each field from the de-reddened $K$ limiting magnitude $M_{K}^{\mathrm{c}}-A_{K}$ and the age of the Herbig AeBe star for two values of $A_{K}=0$ and 2 mag.

\section{References}

Acker A., Chopinet M., Pottasch S.R., Senholm B., 1987, A\&AS 71, 163

Acker A., Senholm B., 1990, A\&AS 86, 219

Ageorges N., Eckart A., Monin J.-L., Ménard F., 1997, 326, 632

van den Ancker M.E., Thé P.S., Tjin A Djie H.R.E., et al., 1997, A\&A 324, L33

van den Ancker M.E., de Winter D., Tjin A Djie H.R.E., 1998, A\&A 330, 145

Anglada G., Rodriguez L.F., Girart J.M., Estarella R., Torrelles J.M., 1994, ApJ 420, 91

Aspin C., Sandell G., Weintraub D.A., 1994, A\&A 282, L25

Bastian U., Mundt R., 1979, A\&AS 36, 57

Berrilli F., Corciulo G., Ingrosso G., Lorenzetti D., Nisini B., Strafella F., 1992, ApJ 398, 254

Bessel M.S., 1979, PASP 91, 589

Bessel M.S., 1991, AJ 101, 662

Bessel M.S., Bret J.M., 1988, PASP 100, 1134

Cantó J., Rodriguez L.F., Calvet N., Levreault R.M., 1984, ApJ 282, 631

Carpenter J.M., Meyer M.R., Dougados C., Strom S.E., Hillenbrand L.A., 1997, AJ 114, 198

Chavarria-K. C., 1985, A\&A 148, 317

Chavarria-K. C., de Lara E., Finkenzeller U., Mendoza E.E., Ocegueda J., 1988, A\&A 197, 151

Close L.M., Roddier F., Hora J.L., et al., 1997, ApJ 489, 210

Cohen M., 1973, MNRAS 161, 97

Cohen M., 1977, ApJ 215, 533

Cohen M., 1980, AJ 85, 29

Cohen M., Kuhi L.V., 1976, ApJ 210, 365

Cohen M., Kuhi L.V., 1979, ApJS 41, 743

Corcoran M., Ray T.P., 1998, A\&A 331, 147

Damiani F., Micela G., Sciortino S., Harnden F.R.Jr., 1994, ApJ 436, 807

D’Antona F., Mazzitelli I., 1994, ApJS 90, 467

Di Francesco J., Evans N.J.II, Harvey P.M., Mundy L.G., Butner H.M., 1994, ApJ 432, 710

Di Francesco J., Evans N.J.II, Harvey P.M., Mundy L.G., Guilloteau S., Chandler C.J., 1997, ApJ 482, 433

Estarella R., Mauersberger R., Torrelles J.M., Anglada G., Gomez J.F., Lopez R., Muders D., 1993, ApJ 419, 698

Fiebig D., 1995, A\&A 298, 207

Finkenzeller U., Mundt R., 1984, A\&AS 55, 109

Fuente A., Martín-Pintado J., Bchiller R., Neri R., Palla F., 1998, A\&A 334, 253

Garmany A.G., 1973, AJ 78, 185

Georgelin Y.P., Georgelin Y.M., 1970, A\&A 6, 349

Goodrich R.W., 1986, ApJ 311, 882

Hamann F., Persson F.E., 1992, ApJS 82, 285

Henning Th., Launhardt E., Steinacker J., Thamm E., 1994, A\&A 291, 546

Herbig G.H., Bell K.R., 1988, Bullettin of the Lick Observatory, No. 1111

Herbst W., Miller D.P., Warner J.W., Herzog A., 1982, AJ 87, 98

Hillenbrand L.A., 1995, Ph.D. Thesis, University of Massachusetts

Hillenbrand L.A., Meyer M.R., Strom S.E., 1995, AJ 109, 280

Hillenbrand L.A., Strom S.E., Vrba F.J., Keene J., 1992, ApJ 397,613 
Hunt L.K., Lisi F., Testi L., Baffa C., Borelli S., et al., 1996, A\&AS 115, 181

Hunt L.K., Mannucci F., Testi L., Migliorini S., Stanga R.M., et al., 1998, AJ 115, 2594

Hunt L.K., Testi L., Borelli S., Maiolino R., Moriondo G., 1994, Technical Report 4/94, Arcetri Astrophysical Observatory

Koo B.-C., 1989, ApJ 337, 318

Koornneef J., 1983, A\&A 128, 84

Lagage P.O., Olofsson G., Cabrit S., Cesarsky C.J., Nordh L., Rodriguez-Espinosa J.M., 1993, ApJ 417, L79

Leinert C., Richichi A., Haas M., 1997, A\&A 318, 472

Levreault R., 1988, ApJ 330, 897

Li W., Evans N.J.II, Harvey P.M., Colomé C., 1994, ApJ 433, 199

Lisi F., Hunt L.K., Baffa C., Biliotti V., Bonaccini D., et al., 1996, PASP 108, 364

Mannings V., 1994, MNRAS 271, 587

Mannings V., Koerner D.W., Sargent A.I., 1997, Nat 388, 555

Mannings V., Sargent A.I., 1997, ApJ 490, 792

Meyer M.R., 1996, Ph.D. Thesis, University of Massachusetts

Molinari S., Brand J., Cesaroni R., Palla F., 1996, A\&A 308, 573

Osterloh M., Beckwith S.V.W., 1995, ApJ 439, 288

Natta A., Grinin V.P., Mannings V., Ungerechts H., 1997, ApJ 491, 885

Natta A., Palla F., Butner H.M., Evans N.J.II, Harvey P.M., 1993, ApJ 406, 674

Palla F., Stahler S.W., 1990, ApJ 360, L47

Palla F., Stahler S.W., 1993, ApJ 418, 414

Palla F., Testi L., Hunter T.R., Taylor G.B., Prusti T., et al., 1995, A\&A 293, 521

Perek L., Kohoutek L., 1967, Publ. House Czech. Acad. Sci., 1
Pirzkal N., Spillar E.J., Dyck H.M., 1997, ApJ 481, 392

Racine R., 1968, AJ 73, 233

Sandell G., Weintraub D.A., 1994, A\&A 292, L1

Scarrot S.M., Brosch N., Ward-Thompson D., Warren-Smith R.F., 1986, MNRAS 223, 505

Schmidt-Kaler Th., 1981, in Landolt-Börnstein, Gruppe VI, Bond 2, 1. Publ. Springer

Shevchenko V.S., Grankin K.N., Ibragimov M.A., Melnikov S.Yu., Yakubov S.D., 1993, Ap\&SS 202, 121

Shevchenko V.S., Vitrichenko E.A., 1994, in: "Nature and evolutionary status of Herbig Ae/Be stars", Thé P.S., Pérez M.R., van den Heuvel J.P.E. (eds.), ASP Conf. Ser., 55

Skinner S.L., Brown A., Stewart R.T., 1993, ApJS 87, 217

Testi L., Palla F., Prusti T., Natta A., Maltagliati S., 1997, A\&A 320, 159 (Paper I)

Testi L., Palla F., Natta A., 1998, A\&A (submitted)

Thé P.S., de Winter D., Pérez M.R., 1994, A\&AS 104, 315

Torrelles J.M., Anglada G., Rodriguez L.F., Cantó J., Barral J.F., 1987, A\&A 177, 171

Torrelles J.M., Ho P.T., Anglada G., Rodriguez L.F., Cantó J., Verdes-Montenegro L., 1989, ApJ 346, 756

Torrelles J.M., Rodriguez L.F., Cantó J., Carral P., Marcaide J., Moran J.M., Ho P.T., 1983, ApJ 274, 214

Verdes-Montenegro L., Gómez J.F., Torrelles J.M., Anglada G., Estalella R., López R., 1991, A\&A 244, 214

Yang J., Umemoto T., Iwata T., Fukui Y., 1991, ApJ 373, 137

Wood D.O.S., Myers P.C., Daugherty D.A., 1994, ApJS 95, 457

Wu Y., Huang M., He J., 1996, A\&AS 115, 283

Zijlstra A., Pottasch S., Bignell C., 1990, A\&AS 82, 273

Zinnecker H., Preibisch Th., 1994, A\&A 292, 152 